\title{
Chemoselective synthesis of oligosaccharides of
}

\section{2-deoxy-2-aminosugars}

Aileen F. G. Bongat, Medha N. Kamat, and Alexei V. Demchenko*

Department of Chemistry and Biochemistry, University of Missouri - St. Louis, One University

Boulevard, St. Louis, Missouri 63121, USA

E-mail: demchenkoa@umsl.edu

\section{Contents:}

S-2 Table 1S. Synthesis of benzoxazolyl 2- $N$-substituted-2-deoxy-tri-O-acetyl-1-thioß-D-glucopyranosides

S-3/S-15 additional experimental data for $\mathbf{D}$ and 1-15

S16/S17 Spectra of chloride D

S18/S-25 Spectra of glycosyl donors 1-4

S-26/S-51 Spectra of the disaccharides 9-12

S52/S53 Spectra of the glycosyl acceptor 13

S54/S57 Spectra of $\mathbf{1 4}$ and $\mathbf{1 5}$ obtained via chemoselective pathways 
Table 1S. Synthesis of benzoxazolyl 2- $N$-substituted-2-deoxy-tri-O-acetyl-1-thio-ß-D-

\section{glucopyranosides}

\section{Starting Material}

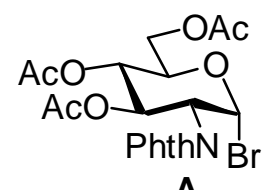

A

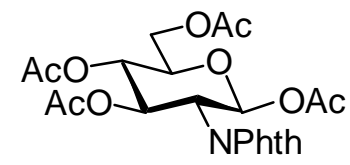

B
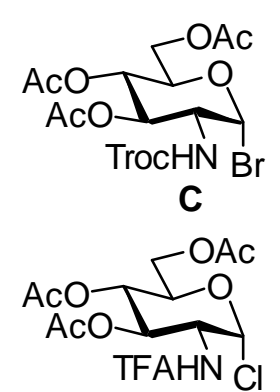

D

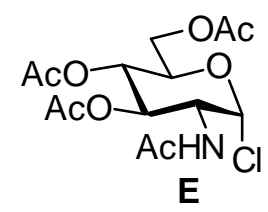

\section{Conditions}

KSBox, 18-crown-6, Acetone, $16 \mathrm{~h}$

HSBox, $\mathrm{BF}_{3}-\mathrm{OEt}_{2}, 3$ ?

$\mathrm{MS}, \mathrm{CH}_{2} \mathrm{Cl}_{2}, 16 \mathrm{~h}$

HSBox, $\mathrm{AlCl}, 3$ ? MS, $\mathrm{CH}_{2} \mathrm{Cl}_{2}, 1.5 \mathrm{~h}$

HSBox, $\mathrm{ZrCh}, 3$ ? MS, $\mathrm{CH}_{2} \mathrm{Cl}_{2}, 16 \mathrm{~h}$

HSBox, TMSOTf, 3 ?

MS, $\mathrm{CH}_{2} \mathrm{Cl}_{2}, 1.5 \mathrm{~h}$

KSBox, 18-crown-6, acetone, $1.5 \mathrm{~h}$

KSBox, 18-crown-6, acetone, $1 \mathrm{~h}$

KSBox, 18-crown-6, acetone, $1 \mathrm{~h}$
Yield $\alpha / \beta$ Ratio

Product
$85 \% \quad \beta$ only

$95 \% \quad 1 / 10$

$75 \% \quad 1 / 16$

$85 \% \quad 1 / 8$

$73 \% \quad \beta$ only

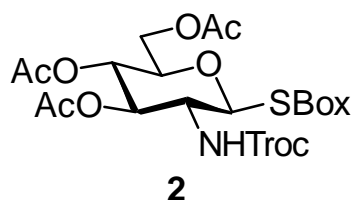

$70 \% \quad \beta$ only
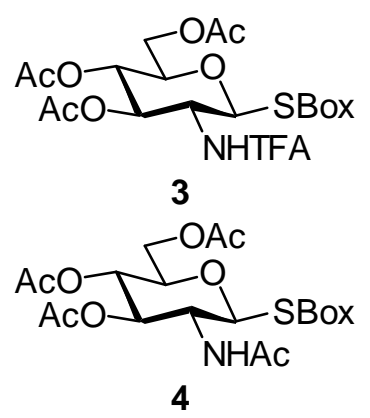


\section{Additional Experimental Data:}

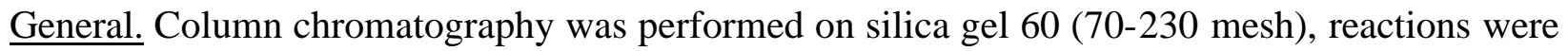
monitored by TLC on Kieselgel $60 \mathrm{~F}_{254}$. The compounds were detected by examination under UV light and by charring with $10 \%$ sulfuric acid in methanol. Solvents were removed under reduced pressure at $<40{ }^{\circ} \mathrm{C} . \mathrm{CH}_{2} \mathrm{Cl}_{2}, \mathrm{ClCH}_{2} \mathrm{CH}_{2} \mathrm{Cl}, \mathrm{CH}_{3} \mathrm{CN}, \mathrm{CHCl}_{3}$, and toluene were distilled from $\mathrm{CaH}_{2}$ directly prior to application. Methanol was dried by refluxing with magnesium methoxide, distilled and stored under argon. Pyridine was dried by refluxing with $\mathrm{CaH}_{2}$ and then distilled and stored over molecular sieves ( $3 \AA$ ). 1,4-Dioxane and acetone were dried and stored over molecular sieves $3 \AA$ and $\mathrm{K}_{2} \mathrm{CO}_{3}$, respectively. Anhydrous DMF was used as is. Molecular sieves ( $3 \AA$ or $4 \AA$ ), used for reactions, were crushed and activated in vacuo at $390{ }^{\circ} \mathrm{C}$ during $8 \mathrm{~h}$ in the first instance and then for $2-3 \mathrm{~h}$ at $390{ }^{\circ} \mathrm{C}$ directly prior to application. AgOTf was coevaporated with toluene $(3 \times 10 \mathrm{~mL})$ and dried in vacuo for $2-3 \mathrm{~h}$ directly prior to application.

${ }^{1} \mathrm{H}$-n.m.r. and two-dimensional spectra were recorded in $\mathrm{CDCl}_{3}$ at $300 \mathrm{MHz},{ }^{13} \mathrm{C}-\mathrm{NMR}$ spectra were recorded in $\mathrm{CDCl}_{3}$ at $75 \mathrm{MHz}$. HRMS determinations were made with the use of JEOL MStation Mass Spectrometer.

\section{3,4,6-tri-O-acetyl-2-deoxy-2-trifluoroacetamido- $\alpha$-D-glucopyranosyl chloride (D). This}

chlorination method was adopted and modified from a procedure previously reported by Pavliak and Kovac. \{Pavliak, V.; Kovac, P., Carbohydr. Res. 1991, 210, 333-337\} To a mixture of 1,3,4,6-tetra-O-acetyl-2-deoxy-2-trifluoroacetamido- $\beta$-D-glucopyranose (4.43 g, $10 \mathrm{mmol})$ and zinc chloride ( $300 \mathrm{mg})$ in alcohol-free chloroform $(15 \mathrm{~mL})$ was added $\alpha, \alpha-2,2-$ dichloromethyl methyl ether (DCMME, $15 \mathrm{~mL}$ ). The mixture was stirred at $50^{\circ} \mathrm{C}$ for an hour, wherein TLC 
showed complete conversion of the starting material into a faster moving product. After concentration of the mixture and co-evaporation with toluene, the residue was purified by column chromatography on silica gel (ethyl acetate - toluene gradient elution) to afford $\mathbf{4}$ as white foam $(3.74 \mathrm{~g}, 89 \%) . \mathrm{R}_{\mathrm{f}}=0.53$ (hexanes-ethyl acetate, $\left.3 / 2, \mathrm{v} / \mathrm{v}\right) ;[\alpha]_{\mathrm{D}}^{25} 32.8^{\circ}(\mathrm{c}=1.00$, $\left.\mathrm{CHCl}_{3}\right) ;{ }^{1} \mathrm{H}-$ n.m.r.: $\delta, 2.04,2.05,2.10\left(3 \mathrm{~s}, 9 \mathrm{H}, 3 \times \mathrm{COCH}_{3}\right), 4.14\left(\mathrm{dd}, 1 \mathrm{H}, J_{5,6 \mathrm{a}}=3.0 \mathrm{~Hz}, J_{6 \mathrm{a}, 6 \mathrm{~b}}=\right.$ 13.6 Hz, H-6a), 4.30-4.35 (m, 2H, H-5, 6b), 4.50-4.56 (m, 1H, H-2), 5.23 (dd, 1H, $J_{4,5}=9.7 \mathrm{~Hz}$ H-4), $5.43\left(\mathrm{dd}, 1 \mathrm{H}, J_{3,4}=10.1 \mathrm{~Hz}, \mathrm{H}-3\right), 6.23\left(\mathrm{~d}, 1 \mathrm{H}, J_{1,2}=3.8 \mathrm{~Hz}, \mathrm{H}-1\right), 7.07$ (d, 1H, NH) ppm,

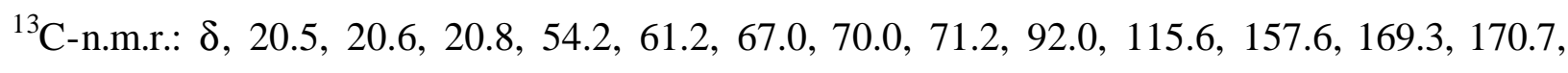
171.7 ppm; HR-FAB MS $[\mathrm{M}+\mathrm{H}]^{+}$calcd for $\mathrm{C}_{14} \mathrm{H}_{18} \mathrm{ClF}_{3} \mathrm{NO}_{8} 420.0673$, found 420.0668 .

\section{Methyl O-(3,4,6-tri-O-acetyl-2-deoxy-2-phthalimido- $\beta$-D-glucopyranosyl)-(1 $\rightarrow$ 6)-2,3,4-tri-}

O-benzyl- $\alpha$-D-glucopyranoside (9a) was obtained from $\mathbf{1}$ and $\mathbf{5}$ as an amorphous solid in $92 \%$ yield. $R_{f}=0.53$ (toluene-ethyl acetate, $\left.3 / 2, \mathrm{v} / \mathrm{v}\right) ;[\alpha]_{\mathrm{D}}^{25} 14.3^{\circ}\left(\mathrm{c}=1.00, \mathrm{CHCl}_{3}\right) ;{ }^{1} \mathrm{H}$-n.m.r.: $\delta$, $1.82,1.99,2.05\left(3 \mathrm{~s}, 9 \mathrm{H}, 3 \times \mathrm{COCH}_{3}\right), 3.13\left(\mathrm{~s}, 3 \mathrm{H}, O C H_{3}\right), 3.20\left(\mathrm{dd}, 1 \mathrm{H}, J_{4,5}=8.9 \mathrm{~Hz}, \mathrm{H}-4\right), 3.35$ $\left(\mathrm{dd}, 1 \mathrm{H}, J_{2,3}=9.7 \mathrm{~Hz}, \mathrm{H}-2\right), 3.59-3.65(\mathrm{~m}, 2 \mathrm{H}, \mathrm{H}-5,6 \mathrm{~b}), 3.80\left(\mathrm{dd}, 1 \mathrm{H}, J_{3,4}=9.3 \mathrm{~Hz}, \mathrm{H}-3\right), 3.81-$ 3.86 (m, 1H, H5'), 4.03-4.16 (m, 3H, H6a, 6b', $\left.\mathrm{CH}_{2}{ }^{a} \mathrm{Ph}\right)$, 4.26-4.40 (m, 4H, H1, 2', 6a', $\left.\mathrm{CH}_{2}{ }^{b} \mathrm{Ph}\right), 4.61\left(\mathrm{dd}, 2 \mathrm{H}, J^{2}=12.1 \mathrm{~Hz}, \mathrm{CH}_{2} \mathrm{Ph}\right), 4.72\left(\mathrm{dd}, 2 \mathrm{H}, J^{2}=10.8 \mathrm{~Hz}, \mathrm{CH}_{2} \mathrm{Ph}\right), 5.14(\mathrm{dd}, 1 \mathrm{H}$, $\left.J_{4^{\prime}, 5^{\prime}}=9.6 \mathrm{~Hz}, \mathrm{H}-4^{\prime}\right), 5.40\left(\mathrm{~d}, 1 \mathrm{H}, J_{1^{\prime}, 2^{\prime}}=8.5 \mathrm{~Hz}, \mathrm{H}-1^{\prime}\right), 5.76\left(\mathrm{dd}, 1 \mathrm{H}, J_{3^{\prime}, 4^{\prime}}=9.1 \mathrm{~Hz}, \mathrm{H}-3^{\prime}\right), 6.97-$ 7.51 (m, 19H, aromatic) ppm, ${ }^{13}$ C-n.m.r.: $\delta, 20.6,20.8,20.9,54.6,55.1,60.5,62.2,68.9,69.1$, $69.3,70.9,72.1,73.5,74.9,75.8,77.7,79.8,82.0,98.1,98.5,123.6$ (x 2), 127.7, 127.8, 127.9 (x 2), 128.1 (x 3), 128.2 (x 2), 128.5 (x 4), 128.6 (x 2), 131.8 (x 2), 134.3 (x 2), 137.9, 138.2, 138.8, 167.8, 169.6, 170.3, 170.9 ppm, HR-FAB MS $[\mathrm{M}+\mathrm{H}]^{+}$calcd for $\mathrm{C}_{48} \mathrm{H}_{51} \mathrm{NO}_{15} \mathrm{Na} 904.3156$, found 904.3157. 
Methyl O-(3,4,6-tri-O-acetyl-2-deoxy-2-trichloroethoxycarbamoyl- $\beta$-D-glucopyranosyl)-

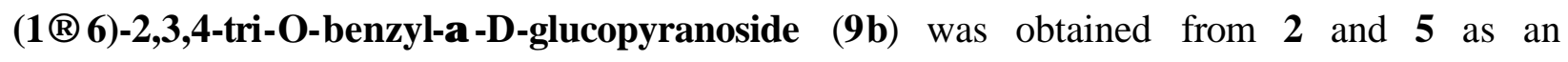
amorphous solid in $91 \%$ yield. $R_{\mathrm{f}}=0.46$ (ethyl acetate-hexanes, $\left.3 / 2, \mathrm{v} / \mathrm{v}\right) ;[\alpha]_{\mathrm{D}}^{25} 22.9^{\circ}(\mathrm{c}=1.00$, $\mathrm{CHCl}_{3}$ ); ${ }^{1} \mathrm{H}$-n.m.r.: $\delta, 1.93,1.95,1.97$ (3s, 9H, 3 x $\left.\mathrm{COCH}_{3}\right), 3.29$ (s, 3H, $\left.\mathrm{OCH}_{3}\right), 3.36-3.43(\mathrm{~m}$, 2H, H-2, 4), 3.51-3.69 (m, 4H, $J_{5}{ }^{\prime}, 6 \mathrm{a}^{\prime}=4.2 \mathrm{~Hz}, \mathrm{H}-2,5,5$ ', 6a), 3.91 (dd, 1H, $\left.J_{3,4} 9.1 \mathrm{~Hz}, \mathrm{H}-3\right)$, 3.98-4.06 (m, 2H, H-6b, 6b'), $4.15\left(\mathrm{dd}, 1 \mathrm{H}, J_{6 \mathrm{a}^{\prime}, 6 \mathrm{~b}^{\prime}}=12.1 \mathrm{~Hz}, \mathrm{H}-6 \mathrm{a}^{\prime}\right), 4.32\left(\mathrm{~d}, 1 \mathrm{H}, J_{1^{\prime}, 2^{\prime}}=8.7 \mathrm{~Hz}\right.$, H-1'), $4.45\left(\mathrm{~s}, 2 \mathrm{H}, \mathrm{CH}_{2} \mathrm{CCl}_{3}\right), 4.52\left(\mathrm{~d}, 1 \mathrm{H}, J_{1,2}=3.5 \mathrm{~Hz}, \mathrm{H} 1\right), 4.65\left(\mathrm{dd}, 2 \mathrm{H}, J^{2}=11.4 \mathrm{~Hz}\right.$, $\left.\mathrm{CH}_{2} \mathrm{Ph}\right), 4.65\left(\mathrm{dd}, 2 \mathrm{H}, J^{2}=12.8 \mathrm{~Hz}, \mathrm{CH}_{2} \mathrm{Ph}\right), 4.79(\mathrm{~d}, 1 \mathrm{H}, \mathrm{NH}), 4.82\left(\mathrm{dd}, 2 \mathrm{H}, J^{2}=10.8 \mathrm{~Hz}\right), 4.95$ $\left(\mathrm{dd}, 1 \mathrm{H}, J_{4^{\prime}, 5^{\prime}}=9.6 \mathrm{~Hz}, \mathrm{H}-4^{\prime}\right), 5.12\left(\mathrm{dd}, 1 \mathrm{H}, J_{3^{\prime}, 4^{\prime}}=9.4 \mathrm{~Hz}, \mathrm{H}-3^{\prime}\right), 7.19-7.30$ (m, 15H, aromatic) ppm, ${ }^{13}$ C-n.m.r.: $\delta, 20.8$ (x 2), 20.9, 55.5, 56.2, 62.2, 68.2, 68.8, 69.7, 72.0, 72.1, 73.6, 74.6, $74.7,76.0,77.4,79.9,82.3,95.4,98.3,101.0,127.9,128.1$ (x 2), 128.2 (x 4), 128.4 (x 2), 128.6 (x 2), 128.7 (x 2), 128.8 (x 2), 138.2, 138.8, 138.9, 154.0, 169.6, 170.8, 170.9 ppm, HR-FAB MS $[\mathrm{M}+\mathrm{H}]^{+}$calcd for $\mathrm{C}_{43} \mathrm{H}_{50} \mathrm{Cl}_{3} \mathrm{NO}_{15} \mathrm{Na}$ 948.2144, found 948.2173.

\section{Methyl O-(3,4,6-tri-O-acetyl-2-deoxy-2-trifluoroacetamido- $\beta$-D-glucopyranosyl)-(1 $\rightarrow$ 6)-}

2,3,4-tri-O-benzyl- $\alpha$-D-glucopyranoside (9c) was obtained from $\mathbf{3}$ and $\mathbf{5}$ as an amorphous solid in $93 \%$ yield. $\mathrm{R}_{\mathrm{f}}=0.48$ (toluene-ethyl acetate, $\left.1 / 1, \mathrm{v} / \mathrm{v}\right) ;[\alpha]_{\mathrm{D}}^{25} 3.51^{\circ}\left(\mathrm{c}=1.00, \mathrm{CHCl}_{3}\right) ;{ }^{1} \mathrm{H}-$ n.m.r.: $\delta, 2.02,2.04,2.06\left(3 \mathrm{~s}, 9 \mathrm{H}, 3\right.$ x $\left.\mathrm{COCH}_{3}\right), 3.34\left(\mathrm{~s}, 3 \mathrm{H}, \mathrm{OCH}_{3}\right), 3.35\left(\mathrm{dd}, 1 \mathrm{H}, J_{4,5}=9.3 \mathrm{~Hz}\right.$, H-4), $3.49\left(\mathrm{dd}, 1 \mathrm{H}, J_{2,3}=9.5 \mathrm{~Hz}, \mathrm{H}-2\right), 3.57\left(\mathrm{dd}, 1 \mathrm{H}, J_{6 \mathrm{a}, 6 \mathrm{~b}}=10.5 \mathrm{~Hz}, \mathrm{H}-6 \mathrm{a}\right), 3.63-3.68(\mathrm{~m}, 1 \mathrm{H}$, $\left.J_{5^{\prime}, 6 \mathrm{a}^{\prime}}=4.3 \mathrm{~Hz}, \mathrm{H}-5^{\prime}\right), 3.74-3.79\left(\mathrm{~m}, 1 \mathrm{H}, J_{5,6 \mathrm{a}}=5.1 \mathrm{~Hz}, \mathrm{H}-5\right), 3.99\left(\mathrm{dd}, 1 \mathrm{H}, J_{3,4}=9.2 \mathrm{~Hz}, \mathrm{H}-3\right)$, 3.97-4.16 (m, 3H, H-2', 6b, 6b'), 4.25 (dd, 1H, $\left.J_{6 \mathrm{a}^{\prime}, 6 \mathrm{~b}^{\prime}}=12.2 \mathrm{~Hz}, \mathrm{H}-6 \mathrm{a}^{\prime}\right), 4.34$ (d, 1H, $J_{1^{\prime}, 2^{\prime}}=8.3$ Hz, H-1'), $4.44\left(\mathrm{dd}, 2 \mathrm{H}, J^{2}=8.3 \mathrm{~Hz}, \mathrm{CH}_{2} \mathrm{Ph}\right), 4.55\left(\mathrm{~d}, 1 \mathrm{H}, J_{1,2}=1.6 \mathrm{~Hz}, \mathrm{H}-1\right), 4.70\left(\mathrm{dd}, 2 \mathrm{H}, J^{2}=\right.$ 
$\left.12.1 \mathrm{~Hz}, \mathrm{CH}_{2} \mathrm{Ph}\right), 4.71\left(\mathrm{dd}, 2 \mathrm{H}, J^{2}=12.1 \mathrm{~Hz}, \mathrm{CH}_{2} \mathrm{Ph}\right), 5.08\left(\mathrm{dd}, 1 \mathrm{H}, J_{4^{\prime}, 5^{\prime}}=9.5 \mathrm{~Hz}, \mathrm{H}-4^{\prime}\right), 5.20$ $\left(\mathrm{dd}, 1 \mathrm{H}, J_{3^{\prime}} 4^{\prime}=10.1 \mathrm{~Hz}, \mathrm{H}-3^{\prime}\right), 6.42(\mathrm{~d}, 1 \mathrm{H}, \mathrm{NH}), 7.25-7.39\left(\mathrm{~m}, 15 \mathrm{H}\right.$, aromatic) ppm, ${ }^{13} \mathrm{C}-$ n.m.r.: $\delta, 20.6,20.8,20.9,54.9,55.3,68.4,68.6,69.5,71.9,72.2,73.6,74.7,75.8,77.4,80.1,82.2,98.1$, $100.5,127.8$ (x 2), 128.0 (x 2), 128.1 (x 6), 128.4 (x 2), 128.6 (x 2), 128.7 (x 4), 138.3, 138.5, $138.9,169.5,170.9,171.3 \mathrm{ppm}$, HR-FAB MS $[\mathrm{M}+\mathrm{H}]^{+}$calcd for $\mathrm{C}_{42} \mathrm{H}_{48} \mathrm{~F}_{3} \mathrm{NO}_{14} \mathrm{Na} 870.2925$, found 870.2911 .

\section{Methyl O-(2-acetamido-3,4,6-tri-O-acetyl-2-deoxy- $\beta$-D-glucopyranosyl)-(1 $\rightarrow$ 6)-2,3,4-tri-O-}

benzyl- $\alpha$-D-glucopyranoside (9d) was obtained from $\mathbf{4}$ and $\mathbf{5}$ as an amorphous solid in $93 \%$ yield. $\mathrm{R}_{\mathrm{f}}=0.56$ (acetone - toluene, $\left.1 / 1, \mathrm{v} / \mathrm{v}\right) ;[\alpha]_{\mathrm{D}}^{25}-20.1^{\mathrm{o}}\left(\mathrm{c}=1.00, \mathrm{CHCl}_{3}\right) ;{ }^{1} \mathrm{H}$-n.m.r.: $\delta, 1.74$, 1.94 (x 3) (4s, $12 \mathrm{H}, 3$ x COCH$\left.H_{3}\right), 3.29\left(\mathrm{~s}, 3 \mathrm{H}, \mathrm{OCH}_{3}\right), 3.40\left(\mathrm{dd}, 1 \mathrm{H}, J_{4,5}=9.3 \mathrm{~Hz}, \mathrm{H}-4\right), 3.44(\mathrm{dd}$, $\left.1 \mathrm{H}, J_{2,3}=9.7 \mathrm{~Hz}, \mathrm{H}-2\right), 3.58-3.82\left(\mathrm{~m}, 4 \mathrm{H}, J_{5^{\prime}, 6 \mathrm{a}^{\prime}}=4.9 \mathrm{~Hz}, \mathrm{H}-2^{\prime}, 5,5^{\prime}, 6 \mathrm{~b}\right), 3.91\left(\mathrm{dd}, 1 \mathrm{H}, J_{3,4}=9.2\right.$ Hz, H-3), 3.97-4.05 (m, 2H, H-6a, 6b'), 4.14 (dd, 1H, $J_{6 a^{\prime}, 6 b^{\prime}}=12.4$ Hz, H-6a'), 4.52 (d, 1H, $J_{1,2}$ $=3.0 \mathrm{~Hz}, \mathrm{H}-1), 4.61\left(\mathrm{~d}, 1 \mathrm{H}, J_{1^{\prime}, 2^{\prime}}=8.1 \mathrm{~Hz}, \mathrm{H}-1^{\prime}\right), 4.63\left(\mathrm{dd}, 2 \mathrm{H}, J^{2}=10.5 \mathrm{~Hz}, \mathrm{CH}_{2} \mathrm{Ph}\right), 4.64(\mathrm{dd}$, $\left.2 \mathrm{H}, J^{2}=12.2 \mathrm{~Hz}, \mathrm{CH}_{2} \mathrm{Ph}\right), 4.81\left(\mathrm{dd}, 2 \mathrm{H}, J^{2}=10.8 \mathrm{~Hz}, \mathrm{CH}_{2} \mathrm{Ph}\right), 4.97\left(\mathrm{dd}, 1 \mathrm{H}, J_{4}, 5^{\prime}=9.7 \mathrm{~Hz}, \mathrm{H}-\right.$ 4'), $5.20\left(\mathrm{dd}, 1 \mathrm{H}, J_{3^{\prime}, 4^{\prime}}=10.4 \mathrm{~Hz}, \mathrm{H}^{\prime} 3^{\prime}\right), 5.36(\mathrm{~d}, 1 \mathrm{H}, \mathrm{NH}), 7.18-7.29(\mathrm{~m}, 15 \mathrm{H}$, aromatic $)$ ppm,

${ }^{13}$ C-n.m.r.: $\delta, 20.8,2.9$ (x 3), 23.5, 54.9, 55.4, 62.3, 67.8, 68.8, 69.7, 72.0, 72.5, 73.5, 74.8, 75.9, $77.6,79.9,82.2,98.2,100.7,127.8,127.9$ (x 2), 128.1, 128.1 (x 2), 128.3 (x 2), 128.6 (x 2), 128.6 (x 2), 128.7 (x 2), 138.3, 138.5, 138.9, 169.6, 170.1, 170.9, 171.0 ppm, HR-FAB MS $[\mathrm{M}+\mathrm{H}]^{+}$calcd for $\mathrm{C}_{42} \mathrm{H}_{51} \mathrm{NO}_{14} \mathrm{Na}$ 816.3207, found 816.3209.

Methyl O-(3,4,6-tri-O-acetyl-2-deoxy-2-phthalimido- $\beta$-D-glucopyranosyl)-(1 $\rightarrow$ 4)-2,3,6-triO-benzyl- $\alpha$-D-glucopyranoside (10a) was obtained from $\mathbf{1}$ and $\mathbf{6}$ as an amorphous solid in $86 \%$ 
yield. $\mathrm{R}_{\mathrm{f}}=0.56$ (toluene-ethyl acetate, $\left.3 / 2, \mathrm{v} / \mathrm{v}\right) ;[\alpha]_{\mathrm{D}}^{25} 11.3^{\circ}\left(\mathrm{c}=1.00, \mathrm{CHCl}_{3}\right) ;{ }^{1} \mathrm{H}-\mathrm{n} . \mathrm{m} . \mathrm{r} .: \delta$, $1.84,2.00($ x 2$)\left(3 \mathrm{~s}, 9 \mathrm{H}, 3 \times \mathrm{COCH}_{3}\right), 3.28\left(\mathrm{~s}, 3 \mathrm{H}, \mathrm{OCH}_{3}\right), 3.34-3.39\left(\mathrm{~m}, 1 \mathrm{H}, J_{5^{\prime}, 6 \mathrm{a}^{\prime}}=3.7 \mathrm{~Hz}\right.$ $\left.J_{5^{\prime}, 6 \mathrm{~b}^{\prime}}=2.0 \mathrm{~Hz}, \mathrm{H} 5^{\prime}\right), 3.45-3.50(\mathrm{~m}, 3 \mathrm{H}, \mathrm{H} 2,6 \mathrm{a}, 6 \mathrm{~b}), 3.55-3.60(\mathrm{~m}, 1 \mathrm{H}, \mathrm{H} 5), 3.82(\mathrm{dd}, 1 \mathrm{H}$, $\left.J_{6 \mathrm{a}^{\prime}, 6 \mathrm{~b}^{\prime}}=10.5 \mathrm{~Hz}, \mathrm{H}-6 \mathrm{~b}^{\prime}\right), 3.90\left(\mathrm{dd}, 1 \mathrm{H}, J_{3,4}=9.1 \mathrm{~Hz}, \mathrm{H}-4\right), 4.00\left(\mathrm{dd}, 1 \mathrm{H}, J_{4,5}=9.30 \mathrm{~Hz}, \mathrm{H}-3\right)$, $4.10\left(\mathrm{dd}, 1 \mathrm{H}, \mathrm{H}-6 \mathrm{a}{ }^{\prime}\right), 4.28\left(\mathrm{dd}, 1 \mathrm{H}, J_{2^{\prime}, 3^{\prime}}=8.4 \mathrm{~Hz}, \mathrm{H}-2^{\prime}\right), 4.36\left(\mathrm{~s}, 2 \mathrm{H}, \mathrm{CH}_{2} \mathrm{Ph}\right), 4.52\left(\mathrm{~d}, 1 \mathrm{H}, J_{1,2}=\right.$ $3.6 \mathrm{~Hz}, \mathrm{H}-1), 4.64\left(\mathrm{dd}, 2 \mathrm{H}, J^{2}=12.2 \mathrm{~Hz}, \mathrm{CH}_{2} \mathrm{Ph}\right), 4.98\left(\mathrm{dd}, 2 \mathrm{H}, J^{2}=11.8 \mathrm{~Hz}, \mathrm{CH}_{2} \mathrm{Ph}\right), 5.13(\mathrm{dd}$, $\left.1 \mathrm{H}, J_{4^{\prime}, 5^{\prime}}=9.6 \mathrm{~Hz}, \mathrm{H}-4^{\prime}\right), 5.65\left(\mathrm{~d}, 1 \mathrm{H}, J_{1^{\prime}, 2^{\prime}}=8.4 \mathrm{~Hz}, \mathrm{H}-1^{\prime}\right), 5.71\left(\mathrm{dd}, 1 \mathrm{H}, J_{3^{\prime}, 4^{\prime}}=9.2 \mathrm{~Hz}, \mathrm{H}-3^{\prime}\right)$, 7.26-7.82 (m, 19H, aromatic) ppm, ${ }^{13}$ C-n.m.r.: $\delta, 20.6,20.8,20.9,55.4,55.5,61.7,68.4,68.8$, $69.5,71.0,71.8,73.0,73.6,74.8,75.7,77.4,79.6,80.4,97.5,98.3,123.8,127.0$ ( х 2), 127.3, 127.5 x2), 127.6, 128.0, 128.2 (x 2), 128.4 (x 8), 128.5 (x 2), 131.6, 134.5, 138.4, 138.5, 139.7, 169.6, 170.4, 170.9 ppm, HR-FAB MS $[\mathrm{M}+\mathrm{H}]^{+}$calcd for $\mathrm{C}_{48} \mathrm{H}_{51} \mathrm{NO}_{15} \mathrm{Na}$ 904.3156, found 904.3141.

\section{Methyl O-(3,4,6-tri-O-acetyl-2-deoxy-2-trichloroethoxycarbamoyl- $\beta$-D-glucopyranosyl)-} $(1 \rightarrow 4)$-2,3,6-tri-O-benzyl- $\alpha$-D-glucopyranoside $(10 b)$ was obtained from 2 and 6 as an amorphous solid in $89 \%$ yield. $R_{\mathrm{f}}=0.55$ (toluene-ethyl acetate, $\left.3 / 2, \mathrm{v} / \mathrm{v}\right) ;[\alpha]_{\mathrm{D}}^{25}-26.3^{\circ}(\mathrm{c}=1.00$, $\mathrm{CHCl}_{3}$ ); ${ }^{1} \mathrm{H}$-n.m.r.: $\delta, 1.93,1.98,2.02\left(3 \mathrm{~s}, 9 \mathrm{H}, 3 \times \mathrm{COCH}_{3}\right), 3.35$ (s, 3H, $\left.\mathrm{OCH}_{3}\right), 3.36-3.72(\mathrm{~m}$, 6H, H-2, 2', 4, 5', 6a, 6b'), 3.79-3.81 (m, 4H, H-3, 5, 6b, $\left.\mathrm{CH}_{2}{ }^{\mathrm{a}} \mathrm{CCl}_{3}\right), 4.11-4.23$ (m, 2H, H-6a', $\left.\mathrm{CH}_{2}{ }^{\mathrm{b}} \mathrm{CCl}_{3}\right), 4.58\left(\mathrm{~d}, 1 \mathrm{H}, J_{1,2}=4.0 \mathrm{~Hz}, \mathrm{H}-1\right), 4.60\left(\mathrm{dd}, 2 \mathrm{H}, J^{2}=12.2 \mathrm{~Hz}, \mathrm{CH}_{2} \mathrm{Ph}\right), 4.59-5.03(\mathrm{~m}$, 8H, H-1', 3', 4', NH, 2 x CH $2 \mathrm{Ph}$ ), 7.14-7.54 (m, 15H, aromatic) ppm, ${ }^{13} \mathrm{C}-$ n.m.r.: $\delta, 20.8$ (x 3), $55.5,56.4,62.0,67.4,68.82,69.3,71.4,72.5,73.7,73.9,74.6,75.4,77.4,77.5,78.8,80.4,95.8$, 98.6, 100.8, 127.3 (x 3), 128.0, 128.2 (x 2), 128.3 (x 2), 128.5 (x 2), 129.3 (x 2), 129.4, 129.7, 
$137.6,138.4,139.8,154.0,169.6,170.5,170.8$ ppm, HR-FAB MS $[\mathrm{M}+\mathrm{H}]^{+}$calcd for $\mathrm{C}_{43} \mathrm{H}_{50} \mathrm{Cl}_{3} \mathrm{NO}_{15} \mathrm{Na}$ 948.2144, found 948.2167.

\section{Methyl O-(3,4,6-tri-O-acetyl-2-deoxy-2-trifluoroacetamido- $\beta$-D-glucopyranosyl)-(1 $\rightarrow$ 4)-}

2,3,6-tri-O-benzyl- $\alpha$-D-glucopyranoside (10c) was obtained from 3 and $\mathbf{6}$ as an amorphous solid in $86 \%$ yield. $\mathrm{R}_{\mathrm{f}}=0.48$ (toluene-ethyl acetate, $\left.3 / 2, \mathrm{v} / \mathrm{v}\right) ;[\alpha]_{\mathrm{D}}^{25}-45.4^{\circ}\left(\mathrm{c}=1.00, \mathrm{CHCl}_{3}\right)$; ${ }^{1} \mathrm{H}$-n.m.r.: $\delta, 1.94,1.99,2.03\left(3 \mathrm{~s}, 9 \mathrm{H}, 3 \times \mathrm{COCH}_{3}\right), 3.36\left(\mathrm{~s}, 3 \mathrm{H}, \mathrm{OCH}_{3}\right), 3.36-3.39\left(\mathrm{~m}, 1 \mathrm{H}, J_{5}, 6 \mathrm{a}\right.$ $\left.=4.2 \mathrm{~Hz}, \mathrm{H} 5^{\prime}\right), 3.44-3.62$ (m, 4H, H-2, 5, 6a, 6b), 3.81-3.93 (m, 4H, H-2', 3, 4, 6b'), 4.14 (dd, $\left.1 \mathrm{H}, J_{6 \mathrm{a}^{\prime}, 6 \mathrm{~b}^{\prime}}=12.4 \mathrm{~Hz}, \mathrm{H}-6 \mathrm{a}^{\prime}\right), 4.28\left(\mathrm{~d}, 1 \mathrm{H}, J_{1^{\prime}, 2^{\prime}}=8.3 \mathrm{~Hz}, \mathrm{H}-1^{\prime}\right), 4.57\left(\mathrm{~d}, 1 \mathrm{H}, J_{1,2}=3.2 \mathrm{~Hz}, \mathrm{H}-1\right)$, $4.59\left(\mathrm{dd}, 2 \mathrm{H}, J^{2}=12.1 \mathrm{~Hz}, \mathrm{CH}_{2} \mathrm{Ph}\right), 4.65\left(\mathrm{dd}, 2 \mathrm{H}, J^{2}=12.3 \mathrm{~Hz}, \mathrm{CH}_{2} \mathrm{Ph}\right), 4.81\left(\mathrm{dd}, 1 \mathrm{H}, J_{3^{\prime}, 4^{\prime}}=9.9\right.$ Hz, H-3'), $4.86\left(\mathrm{dd}, 2 \mathrm{H}, J^{2}=10.9 \mathrm{~Hz}, \mathrm{CH}_{2} \mathrm{Ph}\right), 4.99\left(\mathrm{dd}, 1 \mathrm{H}, J_{4^{\prime}, 5^{\prime}}=9.7 \mathrm{~Hz}, \mathrm{H}-4^{\prime}\right), 5.82(\mathrm{~d}, 1 \mathrm{H}$, $\mathrm{NH}), 7.25-7.49$ (m, 15H, aromatic) ppm, ${ }^{13} \mathrm{C}$-n.m.r.: $\delta, 20.5,20.7,20.8,55.1,55.6,61.9,67.7$, $67.8,68.2,69.3,71.6,72.0,72.2,73.6,73.9,75.3,77.4,78.9,80.0,98.5,99.6,115.7,127.4$ (x 3), 129.0, 128.3 (x 2), 128.5 (x 2), 129.1 (x 2), 129.3 (x 3), 137.5, 138.3, 139.6, 157.2, 169.4, 170.8, 170.9 ppm, HR-FAB MS $[\mathrm{M}+\mathrm{H}]^{+}$calcd for $\mathrm{C}_{42} \mathrm{H}_{48} \mathrm{~F}_{3} \mathrm{NO}_{14} \mathrm{Na}$ 870.2925, found 870.2907.

\section{Methyl O-(3,4,6-tri-O-acetyl-2-deoxy-2-phthalimido- $\beta$-D-glucopyranosyl)-(1 $\rightarrow$ 3)-2,4,6-tri-} O-benzyl- $\alpha$-D-glucopyranoside (11a) was obtained from $\mathbf{1}$ and $\mathbf{7}$ as a colorless syrup in 87\% yield. $\mathrm{R}_{\mathrm{f}}=0.55$ (toluene-ethyl acetate, $\left.3 / 2, \mathrm{v} / \mathrm{v}\right) ;[\alpha]_{\mathrm{D}}^{25}+2.00^{\circ}\left(\mathrm{c}=1.00, \mathrm{CHCl}_{3}\right) ;{ }^{1} \mathrm{H}$-n.m.r.: $\delta$, $1.88,1.98,2.02\left(3 \mathrm{~s}, 9 \mathrm{H}, 3 \times \mathrm{COCH}_{3}\right), 3.07\left(\mathrm{~s}, 3 \mathrm{H}, \mathrm{OCH}_{3}\right), 3.19\left(\mathrm{dd}, 1 \mathrm{H}, J_{2,3}=9.6 \mathrm{~Hz}, \mathrm{H} 2\right)$, 3.45-3.60 (m, 4H, H-4, 5, 6a, 6b), 3.76-3.86 (m, 1H, $\left.J_{5^{\prime}, 6 \mathrm{a}^{\prime}}=1.8 \mathrm{~Hz}, \mathrm{H}-5^{\prime}\right), 4.02\left(\mathrm{dd}, 1 \mathrm{H}, J_{6 \mathrm{a}^{\prime}, 6 \mathrm{~b}^{\prime}}=\right.$ $12.2 \mathrm{~Hz}, \mathrm{H}-6 \mathrm{a}$ '), 4.09 (d, 1H, $\left.J_{1,2}=3.6 \mathrm{~Hz}, \mathrm{H}-1\right), 4.25-4.39$ (m, 3H, H-2, 3, 6b'), 4.22 (dd, 2H, $J^{2}$ $\left.=12.6 \mathrm{~Hz}, \mathrm{CH}_{2} \mathrm{Ph}\right), 4.43\left(\mathrm{dd}, 2 \mathrm{H}, J^{2}=12.3 \mathrm{~Hz}, \mathrm{CH}_{2} \mathrm{Ph}\right), 4.71\left(\mathrm{dd}, 2 \mathrm{H}, J^{2}=10.9 \mathrm{~Hz}, \mathrm{CH}_{2} \mathrm{Ph}\right)$, 
$5.19\left(\mathrm{dd}, 1 \mathrm{H}, J_{4^{\prime}, 5^{\prime}}=9.6 \mathrm{~Hz}, \mathrm{H}-4^{\prime}\right), 5.81\left(\mathrm{~d}, 1 \mathrm{H}, J_{1^{\prime}, 2^{\prime}}=8.4 \mathrm{~Hz}, \mathrm{H}-1^{\prime}\right), 5.97\left(\mathrm{dd}, 1 \mathrm{H}, J_{3^{\prime}, 4^{\prime}}=9.1\right.$

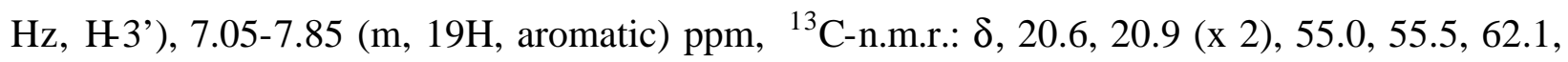
68.6, 69.3, 69.6, 70.7, 71.5, 73.6, 74.1, 74.8, 76.0, 78.4, 81.0, 97.7, 98.2, 123.7, 127.6, 127.9, 128.1 (x 4), 128.2, 128.3 (x 2), 128.4 (x 4), 128.5 (x 4), 128.6 (x 2), 131.7, 134.5, 138.0, 138.4, 138.9, 167.7, 169.8, 170.3, 170.9 ppm, HR-FAB MS $[\mathrm{M}+\mathrm{H}]^{+}$calcd for $\mathrm{C}_{48} \mathrm{H}_{51} \mathrm{NO}_{15} \mathrm{Na} 904.3156$, found 904.3193 .

\section{Methyl O-(3,4,6-tri-O-acetyl-2-deoxy-2-trichloroethoxycarbamoyl- $\beta$-D-glucopyranosyl)-} $(\mathbf{1} \rightarrow 3)$-2,4,6-tri-O-benzyl- $\alpha$-D-glucopyranoside $(\mathbf{1 1 b})$ was obtained from $\mathbf{2}$ and $\mathbf{7}$ as a colorless syrup in $90 \%$ yield. $\mathrm{R}_{\mathrm{f}}=0.52$ (toluene-ethyl acetate, $\left.3 / 2, \mathrm{v} / \mathrm{v}\right) ;[\alpha]_{\mathrm{D}}^{25} 9.10^{\circ}\left(\mathrm{c}=1.00, \mathrm{CHCl}_{3}\right) ;{ }^{1} \mathrm{H}-$ n.m.r.: $\delta, 1.89,1.91,1.93\left(3 \mathrm{~s}, 9 \mathrm{H}, 3 \times \mathrm{COCH}_{3}\right), 3.24\left(\mathrm{~s}, 3 \mathrm{H}, \mathrm{OCH}_{3}\right), 3.46-3.65(\mathrm{~m}, 6 \mathrm{H}, \mathrm{H} 2,5$, 5', 6a, 6b, 6b'), 3.76 (dd, 1H, $\left.J_{2}{ }^{\prime}, 3^{\prime}=9.7 \mathrm{~Hz}, \mathrm{H}-2^{\prime}\right), 3.93$ (dd, 1H, $\left.J_{3,4}=12.3 \mathrm{~Hz}, \mathrm{H}-3\right), 4.12-4.20$ (m, 2H, H-4, 6a'), $4.46\left(\mathrm{dd}, 2 \mathrm{H}, J^{2}=12.1 \mathrm{~Hz}, \mathrm{CH}_{2} \mathrm{Ph}\right), 4.59$ (d, 2H, $\left.\mathrm{CH}_{2} \mathrm{CCl}_{3}\right), 4.61\left(\mathrm{dd}, 2 \mathrm{H}, J^{2}=\right.$ $10.6 \mathrm{~Hz}, \mathrm{CH}_{2} \mathrm{Ph}$ ), $4.61-4.78$ (m, 4H, H-1, 1', 3', NH), 4.74 (dd, 2H, J' = 8.6 Hz, $\left.\mathrm{CH}_{2} \mathrm{Ph}\right), 4.98$ (dd, $1 \mathrm{H}, J_{4}{ }^{\prime}{ }^{\prime}=9.6 \mathrm{~Hz}, \mathrm{H}-4^{\prime}$ ), $7.10-7.37$ (m, 15H, aromatic) ppm, ${ }^{13} \mathrm{C}-$ n.m.r.: $\delta, 20.8$ (x 2), 20.9, $55.3,56.6,62.3,68.6,70.0,71.9,72.9,73.2,73.8,74.9,75.1,75.5,77.5,80.1,81.5,95.8,97.2$ 101.7, 127.8, 128.0, 128.2 (x 2), 128.4 (x 6), 128.6 (x 2), 128.8 (x 2), 129.2, 137.8, 138.1, 138.7, 154.6, 169.6, 170.7, 171.0 ppm, HR-FAB MS $[\mathrm{M}+\mathrm{H}]^{+}$calcd for $\mathrm{C}_{43} \mathrm{H}_{50} \mathrm{Cl}_{3} \mathrm{NO}_{15} \mathrm{Na} 948.2144$, found 948.2186.

\section{Methyl O-(3,4,6-tri-O-acetyl-2-deoxy-2-trifluoroacetamido - $\beta$-D-glucopyranosyl)-(1 $\rightarrow$ 3)-}

2,4,6-tri-O-benzyl- $\alpha$-D-glucopyranoside (11c) was obtained from 3 and $\mathbf{7}$ as a colorless syrup in $89 \%$ yield. $R_{f}=0.46($ ethyl acetate-hexane, $3 / 2, \mathrm{v} / \mathrm{v}) ;[\alpha]_{\mathrm{D}}^{25} 14.3^{\circ}\left(\mathrm{c}=1.00, \mathrm{CHCl}_{3}\right) ;{ }^{1} \mathrm{H}-$ 
n.m.r.: $\delta, 1.91,1.92,1.96\left(3 \mathrm{~s}, 9 \mathrm{H}, 9 \times \mathrm{COCH}_{3}\right), 3.20\left(\mathrm{~s}, 3 \mathrm{H}, 3 \times \mathrm{OCH}_{3}\right), 3.41-3.61\left(\mathrm{~m}, 6 \mathrm{H}, J_{5,6 \mathrm{a}}=\right.$ $\left.2.1 \mathrm{~Hz}, \mathrm{H} 2,5^{\prime}, 6 \mathrm{a}, 6 \mathrm{a}^{\prime}, 6 \mathrm{~b}, 6 \mathrm{~b}^{\prime}\right), 3.96$ (dd, 1H, $\left.J_{6 \mathrm{a}, 6 \mathrm{~b}}=12.3 \mathrm{~Hz}, \mathrm{H}-6 \mathrm{a}\right), 4.10$ (dd, 1H, $J_{2^{\prime}, 3^{\prime}}=9.2$ $\left.\mathrm{Hz}, \mathrm{H} 2^{\prime}\right), 4.18\left(\mathrm{dd}, 1 \mathrm{H}, J_{3,4}=9.1 \mathrm{~Hz}, \mathrm{H}-3\right), 4.20\left(\mathrm{dd}, 1 \mathrm{H}, \mathrm{H} 6 \mathrm{a}^{\prime}\right), 4.44\left(\mathrm{dd}, 2 \mathrm{H}, J^{2}=12.2 \mathrm{~Hz}\right.$ $\left.\mathrm{CH}_{2} \mathrm{Ph}\right), 4.53\left(\mathrm{~s}, 2 \mathrm{H}, \mathrm{CH}_{2} \mathrm{Ph}\right), 4.57\left(\mathrm{~d}, 1 \mathrm{H}, J_{1,2}=3.4 \mathrm{~Hz}, \mathrm{H} 1\right), 4.60\left(\mathrm{dd}, 2 \mathrm{H}, J^{2}=11.0 \mathrm{~Hz}\right.$, $\left.\mathrm{CH}_{2} \mathrm{Ph}\right), 4.90\left(\mathrm{dd}, 1 \mathrm{H}, J_{3^{\prime}, 4^{\prime}}=10.6 \mathrm{~Hz}, \mathrm{H}-3^{\prime}\right), 4.95\left(\mathrm{~d}, 1 \mathrm{H}, J_{1^{\prime}, 2^{\prime}}=8.8 \mathrm{~Hz}, \mathrm{H}-1^{\prime}\right), 5.05(\mathrm{dd}, 1 \mathrm{H}$, $\left.J_{4^{\prime}, 5^{\prime}}=9.6 \mathrm{~Hz}, \mathrm{H}-4^{\prime}\right), 6.09$ (d, 1H, NH), 7.10-7.38 (m, 15H, aromatic) ppm, ${ }^{13} \mathrm{C}-$ n.m.r.: $\delta, 20.9$, 21.0, 21.1, 55.2, 55.4, 62.3, 68.4, 68.7, 70.2, 72.3, 72.7, 72.9, 74.0, 75.3, 75.7, 77.7, 80.0, 81.5, 97.4, 101.1, 127.8 (x 2), 128.0, 128.2, 128.4 (x 2), 128.5 (x 2), 128.6 (x 2), 128.8 (x 2), 128.9, 129.3 (x 2), 138.1, 138.3, 138.9, 157.7, 169.7, 171.1, 171.3 ppm, HR-FAB MS $[M+H]^{+}$calcd for $\mathrm{C}_{42} \mathrm{H}_{48} \mathrm{~F}_{3} \mathrm{NO}_{14} \mathrm{Na} 870.2925$, found 870.2941.

\section{Methyl O-(3,4,6-tri-O-acetyl-2-deoxy-2-phthalimido- $\beta$-D-glucopyranosyl)-(1 $\rightarrow 2)$-2,4,6-tri-}

O-benzyl- $\alpha$-D-glucopyranoside (12a) was obtained from $\mathbf{1}$ and $\mathbf{8}$ as an amorphous solid in $92 \%$ yield. $R_{\mathrm{f}}=0.50$ (toluene-ethyl acetate, $\left.3 / 2, \mathrm{v} / \mathrm{v}\right) ;[\alpha]_{\mathrm{D}}^{25} 57.8^{\circ}\left(\mathrm{c}=1.00, \mathrm{CHCl}_{3}\right) ;{ }^{1} \mathrm{H}$-n.m.r.: $\delta$, 1.74, 1.96, 2.05 (3s, 9H, 3 x $\mathrm{COCH}_{3}$ ), 3.33 (s, 3H, $\mathrm{OCH}_{3}$ ), 3.48-3.65 (m, 5H, H-2, 4, 5, 6a, 6b), 3.77 (dd, $\left.1 \mathrm{H}, J_{3,4}=9.3 \mathrm{~Hz}, \mathrm{H}-3\right), 3.80-3.84(\mathrm{~m}, 1 \mathrm{H}, \mathrm{H}-5$ '), 4.15-4.18 (m, 2H, H-6a', 6b'), 4.30 $\left(\mathrm{dd}, 2 \mathrm{H}, J^{2}=10.7 \mathrm{~Hz}, \mathrm{CH}_{2} \mathrm{Ph}\right), 4.31\left(\mathrm{~s}, 2 \mathrm{H}, \mathrm{CH}_{2} \mathrm{Ph}\right), 4.41-4.44\left(\mathrm{~m}, 1 \mathrm{H}, \mathrm{H}-2\right.$ '), 4.46 (dd, 2H, $J^{2}=$ $\left.12.2 \mathrm{~Hz}, \mathrm{CH}_{2} \mathrm{Ph}\right), 4.94\left(\mathrm{~d}, 1 \mathrm{H}, J_{1,2}=3.3 \mathrm{~Hz}, \mathrm{H}-1\right), 5.08\left(\mathrm{dd}, 1 \mathrm{H}, J_{4,5}=9.6 \mathrm{~Hz}, \mathrm{H}-4\right.$ '), $5.56(\mathrm{~d}, 1 \mathrm{H}$, $\left.J_{1^{\prime}, 2^{\prime}}=8.5 \mathrm{~Hz}, \mathrm{H}-1^{\prime}\right), 5.66\left(\mathrm{dd}, 1 \mathrm{H}, J_{3^{\prime}, 4^{\prime}}=9.2 \mathrm{~Hz}, \mathrm{H} 3^{\prime}\right), 6.73-7.40(\mathrm{~m}, 19 \mathrm{H}$, aromatic $)$ ppm, ${ }^{13}$ C-n.m.r.: $\delta, 20.6,20.8,21.0,54.8,56.3,62.6,68.5,69.0,70.1,71.0,72.2,73.6,74.8,75.1$, $77.8,80.5,83.1,99.3,100.0,123.5,126.1$ (x 2), 126.7, 127.7, 127.9, 128.0 (x 4), 128.1 (x 6), 128.3 (x 2), 128.5 (x 2), 131.0, 134.2 (x 2), 138.1 (x 2), 138.7, 169.7, 170.3, 170.8 ppm, HRFAB MS [M+H] $]^{+}$calcd for $\mathrm{C}_{48} \mathrm{H}_{51} \mathrm{NO}_{15} \mathrm{Na} 904.3156$, found 904.3130 . 
Methyl O-(3,4,6-tri-O-acetyl-2-deoxy-2-trichloroethoxycarbamoyl- $\beta$-D-glucopy ranosyl)$(1 \rightarrow 2)-3,4,6$-tri-O-benzyl- $\alpha$-D-glucopyranoside (12b) was obtained from 2 and 8 as an amorphous solid in $92 \%$ yield. $R_{f}=0.49$ (toluene-ethyl acetate, $\left.3 / 2, v / v\right) ;[\alpha]_{D}^{25} 43.3^{\circ}(c=1.00$, $\mathrm{CHCl}_{3}$ ); ${ }^{1} \mathrm{H}$-n.m.r.: $\delta, 1.90,1.94,2.01$ (3s, 9H, 3 x $\left.\mathrm{COCH}_{3}\right), 3.32$ (s, 3H, $\left.\mathrm{OCH}_{3}\right), 3.56-3.70(\mathrm{~m}$, 7H, H-2, 2', 4, 5, 5', 6a, 6b), 3.89 (dd, 1H, J3,4 = 9.3 Hz, H-3), 4.07-4.16 (m, 2H, H-6a', 6b'), $4.24\left(\mathrm{dd}, 2 \mathrm{H}, J^{2}=12.2 \mathrm{~Hz}, \mathrm{CH}_{2} \mathrm{CCl}_{3}\right), 4.49\left(\mathrm{dd}, 2 \mathrm{H}, J^{2}=12.1 \mathrm{~Hz}, \mathrm{CH}_{2} \mathrm{Ph}\right), 4.50\left(\mathrm{dd}, 2 \mathrm{H}, J^{2}=\right.$ $\left.10.8 \mathrm{~Hz}, \mathrm{CH}_{2} \mathrm{Ph}\right), 4.71-4.79$ (m, 4H, H1', NH, $\left.\mathrm{CH}_{2} \mathrm{Ph}\right), 4.85$ (d, 1H, J1,2 = 3.6 Hz, H1), 4.94 $\left(\mathrm{dd}, 1 \mathrm{H}, J_{4^{\prime}, 5^{\prime}}=9.7 \mathrm{~Hz}, \mathrm{H}-4^{\prime}\right), 5.14\left(\mathrm{dd}, 1 \mathrm{H}, J_{3^{\prime}, 4^{\prime}}=9.9 \mathrm{~Hz}, \mathrm{H}-3^{\prime}\right), 6.95-7.29$ (m, 15H, aromatic) ppm, ${ }^{13}$ C-n.m.r.: $\delta, 20.8$ (x 2), 21.0, 29.9, 55.4, 56.5, 62.4, 68.5, 68.9, 70.2, 72.1 (х 2), 73.7, $75.2,75.3,77.4,78.2,81.3,81.4,95.5,99.5,101.9,127.1,127.8,127.9,128.0,128.1$ (x 4), 128.5 (x 2), 128.6 (x 2), 128.7 (x 2), 138.1, 138.2, 139.1, 154.0, 169.7, 170.5, 170.8 ppm, HR-FAB MS $[\mathrm{M}+\mathrm{H}]^{+}$calcd for $\mathrm{C}_{43} \mathrm{H}_{50} \mathrm{Cl}_{3} \mathrm{NO}_{15} \mathrm{Na}$ 948.2144, found 948.2172.

\section{Methyl O-(3,4,6-tri-O-acetyl-2-deoxy-2-trifluoroacetamido- $\beta$-D-glucopyranosyl)-(1 $\rightarrow 2)$ -}

3,4,6-tri-O-benzyl- $\alpha$-D-glucopyranoside (12c) was obtained from $\mathbf{3}$ and $\mathbf{8}$ as an amorphous solid in $84 \%$ yield. $\mathrm{R}_{\mathrm{f}}=0.45$ (toluene-ethyl acetate, $\left.1 / 1, \mathrm{v} / \mathrm{v}\right) ;[\alpha]_{\mathrm{D}}^{25} 8.6^{\circ}\left(\mathrm{c}=1.0, \mathrm{CHCl}_{3}\right) ;{ }^{1} \mathrm{H}-$ n.m.r.: $\delta, 1.95,2.02,2.08\left(3 \mathrm{~s}, 9 \mathrm{H}, 3 \mathrm{x} \mathrm{COCH}_{3}\right), 3.37$ (s, 3H, OCH$), 3.60-3.75(\mathrm{~m}, 6 \mathrm{H}, \mathrm{H}-2,4,5$, 5', 6a, 6b), $3.93\left(\mathrm{dd}, 1 \mathrm{H}, J_{3,4}=9.2 \mathrm{~Hz}, \mathrm{H}-3\right), 4.06\left(\mathrm{dd}, 1 \mathrm{H}, J_{2}^{\prime}, 3^{\prime}=8.8 \mathrm{~Hz}, \mathrm{H}-2^{\prime}\right), 4.14-4.24$ (m, 2H, H-6a', 6b'), 4.52 (dd, 2H, $\left.J^{2}=10.6 \mathrm{~Hz}, \mathrm{CH}_{2} \mathrm{Ph}\right), 4.54\left(\mathrm{dd}, 2 \mathrm{H}, J^{2}=11.7 \mathrm{~Hz}, \mathrm{CH}_{2} \mathrm{Ph}\right), 4.74$ $\left(\mathrm{dd}, 2 \mathrm{H}, J^{2}=10.6 \mathrm{~Hz}, \mathrm{CH}_{2} \mathrm{Ph}\right), 4.88\left(\mathrm{~d}, 1 \mathrm{H}, J_{1^{\prime}, 2^{\prime}}=7.9 \mathrm{~Hz}, \mathrm{H}-1^{\prime}\right), 4.90$ (d, 1H, $J_{1,2}=3.2 \mathrm{~Hz}, \mathrm{H}-$ 1), $5.06\left(\mathrm{dd}, 1 \mathrm{H}, J_{4^{\prime}, 5^{\prime}}=9.6 \mathrm{~Hz}, \mathrm{H} 4^{\prime}\right), 5.20\left(\mathrm{dd}, 1 \mathrm{H}, J_{3^{\prime}, 4^{\prime}}=9.9 \mathrm{~Hz}, \mathrm{H} 3^{\prime}\right), 6.33(\mathrm{~d}, 1 \mathrm{H}, \mathrm{NH})$,

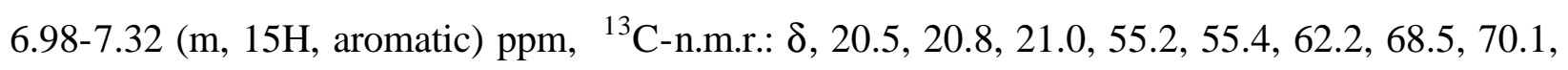


72.1, 72.3, 73.7, 75.1, 75.2, 77.4, 78.3, 81.0 (x 2), 99.5, 101.2, 113.6, 117.4, 127.0 (x 2), 127.7, $127.9,128.1$ (x 2), 128.5 (x 4), 128.6 (x 2), 128.7 (x 2), 138.0, 138.1, 138.7, 157.4, 169.5, 170.8, 171.0 ppm, HR-FAB MS [M+H] $]^{+}$calcd for $\mathrm{C}_{42} \mathrm{H}_{48} \mathrm{~F}_{3} \mathrm{NO}_{14} \mathrm{Na}$ 870.2925, found 870.2911.

Benzoxazolyl 3,4-di- $O$-acetyl-2-deoxy-2-phthalimido-1-thio- $\beta$-D-glucopyranoside (13). To a suspension of the SBox glycoside $1(0.57 \mathrm{~g}, 1.0 \mathrm{mmol})$ in methanol $(10 \mathrm{~mL})$ was added a $0.1 \mathrm{~N}$ solution of $\mathrm{NaOCH}_{3}$ in methanol to $\mathrm{pH}$ 7.5. The reaction mixture was kept for $4 \mathrm{~h}$ at $\mathrm{rt}$, then Dowex $\left(\mathrm{H}^{+}\right)$was added until neutral $\mathrm{pH}$. The resin was filtered off and washed with methanol $(5$ x $15 \mathrm{~mL}$ ). The combined filtrate was concentrated in vacuo and dried. A crude residue containing benzoxazolyl 2-deoxy-2-phthalimido-1-thio- $\beta$-D-glucopyranoside (44 mg, $1.0 \mathrm{mmol}$ ), was dissolved in dry pyridine $(5 \mathrm{~mL})$ and 4dimethylaminopyridine $(0.24 \mathrm{~g}, 2.0 \mathrm{mmol})$ along with triphenylmethyl chloride $(1.1 \mathrm{~g}, 4.0 \mathrm{mmol})$ was added. The reaction mixture was refluxed at $55{ }^{\circ} \mathrm{C}$ for $24 \mathrm{~h}$, then cooled to $\mathrm{rt}$ and acetic anhydride $(0.95 \mathrm{~mL}, 10 \mathrm{mmol})$ was added. The reaction mixture was stirred at $\mathrm{rt}$ for $1 \mathrm{~h}$ and then quenched with methanol $(10 \mathrm{~mL})$. Volatile compounds were evaporated in vacuo, the residue was diluted with $\mathrm{CH}_{2} \mathrm{Cl}_{2}(100 \mathrm{~mL})$, and the organic layer was washed with water $(20 \mathrm{~mL})$, saturated aq. $\mathrm{NaHCO}_{3}(20 \mathrm{~mL})$, water $(20 \mathrm{~mL})$, $1 \mathrm{M} \mathrm{HCl}(2 \times 20 \mathrm{~mL})$, and water $(3 \times 20 \mathrm{~mL})$. The organic phase was separated, dried over $\mathrm{MgSO}_{4}$, and concentrated in vacuo. A crude residue was then dissolved in $\mathrm{CH}_{2} \mathrm{Cl}_{2}$ containing trifluoroacetic acid, and water (94/5/1, v/v/v, respectively). The reaction mixture was kept for $2 \mathrm{~h}$ at rt, then diluted with $\mathrm{CH}_{2} \mathrm{Cl}_{2}(100 \mathrm{~mL})$ and washed with water $(20 \mathrm{~mL})$, sat. $\mathrm{NaHCO}_{3}(20 \mathrm{~mL})$, and water $(3 \times 20 \mathrm{~mL})$. The organic phase was separated, dried over $\mathrm{MgSO}_{4}$, and concentrated in vacuo. The residue was purified by column chromatography on silica gel (ethyl acetate - hexane gradient elution) to allow $\mathbf{1 3}$ as an amorphous solid in $62 \%$ yield. $\mathrm{R}_{\mathrm{f}}=0.56$ (toluene-ethyl 
acetate, $2 / 3, v / v) ;[\alpha]_{\mathrm{D}}^{25} 43.0^{\circ}\left(\mathrm{c}=1.00, \mathrm{CHCl}_{3}\right) ;{ }^{1} \mathrm{H}-\mathrm{n} . \mathrm{m} . \mathrm{r} .: \delta, 1.90,2.10\left(2 \mathrm{~s}, 6 \mathrm{H}, 2 \times \mathrm{COCH}_{3}\right)$, $3.68\left(\mathrm{dd}, 1 \mathrm{H}, J_{6 \mathrm{a}, 6 \mathrm{~b}}=12.5 \mathrm{~Hz}, \mathrm{H}-6 \mathrm{a}\right), 3.84(\mathrm{dd}, 1 \mathrm{H}, \mathrm{H}-6 \mathrm{~b}), 3.95-4.01\left(\mathrm{~m}, 1 \mathrm{H}, J_{5,6 \mathrm{a}}=5.1 \mathrm{~Hz}, \mathrm{H}-5\right)$, $4.66\left(\mathrm{dd}, 1 \mathrm{H}, J_{2,3}=10.5 \mathrm{~Hz}, \mathrm{H}-2\right), 5.23\left(\mathrm{dd}, 1 \mathrm{H}, J_{4,5}=9.7 \mathrm{~Hz}, \mathrm{H}-4\right), 6.01\left(\mathrm{dd}, 1 \mathrm{H}, J_{3,4}=10.0 \mathrm{~Hz}\right.$, H-3), $6.50\left(\mathrm{~d}, 1 \mathrm{H}, J_{1,2}=10.7 \mathrm{~Hz}, \mathrm{H} 1\right), 7.26-7.86\left(\mathrm{~m}, 8 \mathrm{H}\right.$, aromatic) ppm, ${ }^{13} \mathrm{C}-$-n.m.r.: $\delta, 20.7$, 20.9, 53.8, 61.7, 69.2, 71.5, 77.5, 79.2, 81. 110.4 (x 2), 119.5 (x 2), 124.1 (x 4), 124.9 (x 2), 134.8 (x 2), 141.8, 152.1, 170.3, 170.4 ppm, HR-FAB MS $[\mathrm{M}+\mathrm{H}]^{+}$calcd for $\mathrm{C}_{25} \mathrm{H}_{23} \mathrm{~N}_{2} \mathrm{O}_{9} \mathrm{~S}$ 527.1124, found 527.1119.

\section{Benzoxazolyl} $O$-(3,4,6-tri- $O$-acetyl-2-deoxy-2-trichloroethoxycarbamoyl- $\beta$-Dglucopyranosyl)-(1 $\rightarrow 6)-3,4-$ di- $O$-acetyl-2-deoxy-2-phthalimido- $\beta$-D-glucopyranoside was obtained by Method B from $\mathbf{2}$ and $\mathbf{1 3}$ as an amorphous solid in $82 \%$ yield: $\mathrm{R}_{\mathrm{f}}=0.57$ (toluene-ethyl acetate, 2/3, v/v); $[\alpha]_{\mathrm{D}}^{25} 61.2^{\mathrm{o}}\left(\mathrm{c}=1.00, \mathrm{CHCl}_{3}\right) ;{ }^{1} \mathrm{H}$-n.m.r.: $\delta, 1.87,1.93,1.96$, 2.07, 2.09 (5s, 15H, 5 x COCH 3 ), 3.54-3.61 (m, 1H, H-5'), 3.78-3.96 (m, 3H, H-2', 6a, 6b), 4.07$4.26\left(\mathrm{~m}, 3 \mathrm{H}, \mathrm{H}-5,6 \mathrm{a}^{\prime}, 6 \mathrm{~b}^{\prime}\right), 4.54\left(\mathrm{dd}, 1 \mathrm{H}, J_{2,3}=10.6 \mathrm{~Hz}, \mathrm{H}-2\right), 4.79$ (d, 1H, $\left.J_{1^{\prime}, 2^{\prime}}=8.5 \mathrm{~Hz}, \mathrm{H}-1^{\prime}\right)$, 4.84-4.92 (m, 3H, H-3', $\left.\mathrm{CH}_{2} \mathrm{CCl}_{3}\right), 4.97-5.10\left(\mathrm{~m}, 2 \mathrm{H}, \mathrm{H}-4,4^{\prime}\right), 5.90$ (dd, $\left.1 \mathrm{H}, J_{3,4}=9.7 \mathrm{~Hz}, \mathrm{H}-3\right)$, $6.41\left(\mathrm{~d}, 1 \mathrm{H}, J_{1,2}=10.9 \mathrm{~Hz}, \mathrm{H} 1\right), 6.84(\mathrm{~d}, 1 \mathrm{H}, \mathrm{NH}), 7.26-7.88\left(\mathrm{~m}, 8 \mathrm{H}\right.$, aromatic) ppm, ${ }^{13} \mathrm{C}-$ n.m.r.: $\delta, 20.6,20.8$ (х 2), 20.9, 21.0, 29.9, 53.6, 55.9, 62.1, 67.9, 68.9, 69.0, 71.2, 72.0, 72.6, $74.8,77.4,79.8,81.7,95.9,101.7,110.5$ (x 2), 119.5 (x 2), 124.1 (x 2), 124.9, 125.3, 134.8, 141.3, 152.0, 155.4, 160.8, 166.6, 169.6, 169.9, 170.1, 170.2, 171.0 ppm, HR-FAB MS $[\mathrm{M}+\mathrm{H}]^{+}$ calcd for $\mathrm{C}_{40} \mathrm{H}_{40} \mathrm{Cl}_{3} \mathrm{~N}_{3} \mathrm{O}_{18} \mathrm{SNa}$ 1010.0991, found 1010.0959.

Methyl $O$-(3,4,6-tri-O-acetyl-2-deoxy-2-trichloroethoxycarbamoyl- $\beta$-D-glucopyranosyl)$(1 \rightarrow 6)$ - $O$-(3,4-di- $O$-acetyl-2-deoxy-2-phthalimido- $\beta$-D-glucopyranosyl)-(1 $\rightarrow$ 6)-2,3,4-tri- $O$ - 
benzyl- $\alpha$-D-glucopyranoside (15) was obtained by Method A from 5 and $\mathbf{1 4}$ as an amorphous solid in $73 \%$ yield: $\mathrm{R}_{\mathrm{f}}=0.56$ (toluene-ethyl acetate, $\left.2 / 3, \mathrm{v} / \mathrm{v}\right) ;[\alpha]_{\mathrm{D}}^{25} 7.30^{\circ}\left(\mathrm{c}=1.00, \mathrm{CHCl}_{3}\right) ;{ }^{1} \mathrm{H}-$ n.m.r.: $\delta, 1.76,1.94,1.95,1.97,2.03\left(5 \mathrm{~s}, 15 \mathrm{H}, 5 \times \mathrm{COCH}_{3}\right), 3.18\left(\mathrm{~s}, 3 \mathrm{H}, \mathrm{OCH}_{3}\right), 3.27(\mathrm{dd}, 1 \mathrm{H}$, $\left.J_{4 \mathrm{~A}, 5 \mathrm{~A}}=9.4 \mathrm{~Hz}, \mathrm{H}-4 \mathrm{~A}\right), 3.36\left(\mathrm{dd}, 1 \mathrm{H}, J_{2 \mathrm{~A}, 3 \mathrm{~A}}=9.6 \mathrm{~Hz}, \mathrm{H}-2 \mathrm{~A}\right), 3.55-3.82\left(\mathrm{~m}, 7 \mathrm{H}, J_{5 \mathrm{~B}, 6 \mathrm{~B}}=1.9 \mathrm{~Hz}\right.$ H-2C, 3A, 5A, 5B, 5C, 6B, 6C'), $3.93\left(\mathrm{dd}, 1 \mathrm{H}, J_{6 \mathrm{~B}, 6 \mathrm{~B}^{\prime}}=10.9 \mathrm{~Hz}, \mathrm{H}-6 \mathrm{~B}^{\prime}\right), 4.00-4.10(\mathrm{~m}, 3 \mathrm{H}, \mathrm{H}-$ $\left.6 \mathrm{~A}^{\prime}, 6 \mathrm{C}, \mathrm{CH}_{2}{ }^{a} \mathrm{Ph}\right), 4.20-4.36\left(\mathrm{~m}, 3 \mathrm{H}, \mathrm{H}-2 \mathrm{~B}, 6 \mathrm{~A}, \mathrm{CH}_{2}{ }^{b} \mathrm{Ph}\right), 4.50-4.71(\mathrm{~m}, 4 \mathrm{H}, \mathrm{H}-1 \mathrm{~A}, 1 \mathrm{C}$, $\left.\mathrm{CH}_{2} \mathrm{CCl}_{3}\right), 4.61\left(\mathrm{~d}, 2 \mathrm{H}, J^{2}=15.8 \mathrm{~Hz}, \mathrm{CH}_{2} \mathrm{Ph}\right), 4.70\left(\mathrm{dd}, 2 \mathrm{H}, J^{2}=10.9 \mathrm{~Hz}, \mathrm{CH}_{2} \mathrm{Ph}\right), 4.93-5.02(\mathrm{~m}$, $2 \mathrm{H}, \mathrm{H}-4 \mathrm{~B}, 4 \mathrm{C}), 5.10\left(\mathrm{dd}, 1 \mathrm{H}, J_{3 \mathrm{C}, 4 \mathrm{C}}=9.9 \mathrm{~Hz}, \mathrm{H}-3 \mathrm{C}\right), 5.38\left(\mathrm{~d}, 1 \mathrm{H}, J_{1 \mathrm{~B}, 2 \mathrm{~B}}=8.5 \mathrm{~Hz}, \mathrm{H}-1 \mathrm{~B}\right), 5.56$ $(\mathrm{d}, 1 \mathrm{H}, \mathrm{NH}), 5.68\left(\mathrm{dd}, 1 \mathrm{H}, J_{3 \mathrm{~B}, 4 \mathrm{~B}}=10.7 \mathrm{~Hz}, \mathrm{H} 3 \mathrm{~B}\right), 6.84-6.90(\mathrm{~m}, 15 \mathrm{H}$, aromatic $)$ ppm; ${ }^{13} \mathrm{C}-$ n.m.r.: $\delta, 20.6,20.8,20.9,20.9,21.0,29.9,54.7,55.3,56.4,62.1,68.4,69.0,69.4,69.6,69.8$, $70.9,72.1,72.7,73.2,73.8,74.8,75.8,77.4,79.6,82.0,95.7,98.1,98.5,101.8,123.7$ (x 2), 127.7 (x 2), 127.8 (x 2), 127.9 (x 2), 128.1 (x 3), 128.3 (x 2), 128.5 (x 2), 128.5 (x 3), 128.7 (x 2), 131.3, 134.4 (x 2), 137.9, 138.3, 139.0, 154.6, 169.7, 170.0, 170.3, 170.7, 170.9 ppm; HRFAB MS $[\mathrm{M}+\mathrm{H}]^{+}$calcd for $\mathrm{C}_{61} \mathrm{H}_{67} \mathrm{Cl}_{3} \mathrm{~N}_{2} \mathrm{O}_{23} \mathrm{Na}$ 1323.3098, found 1323.3146 . 

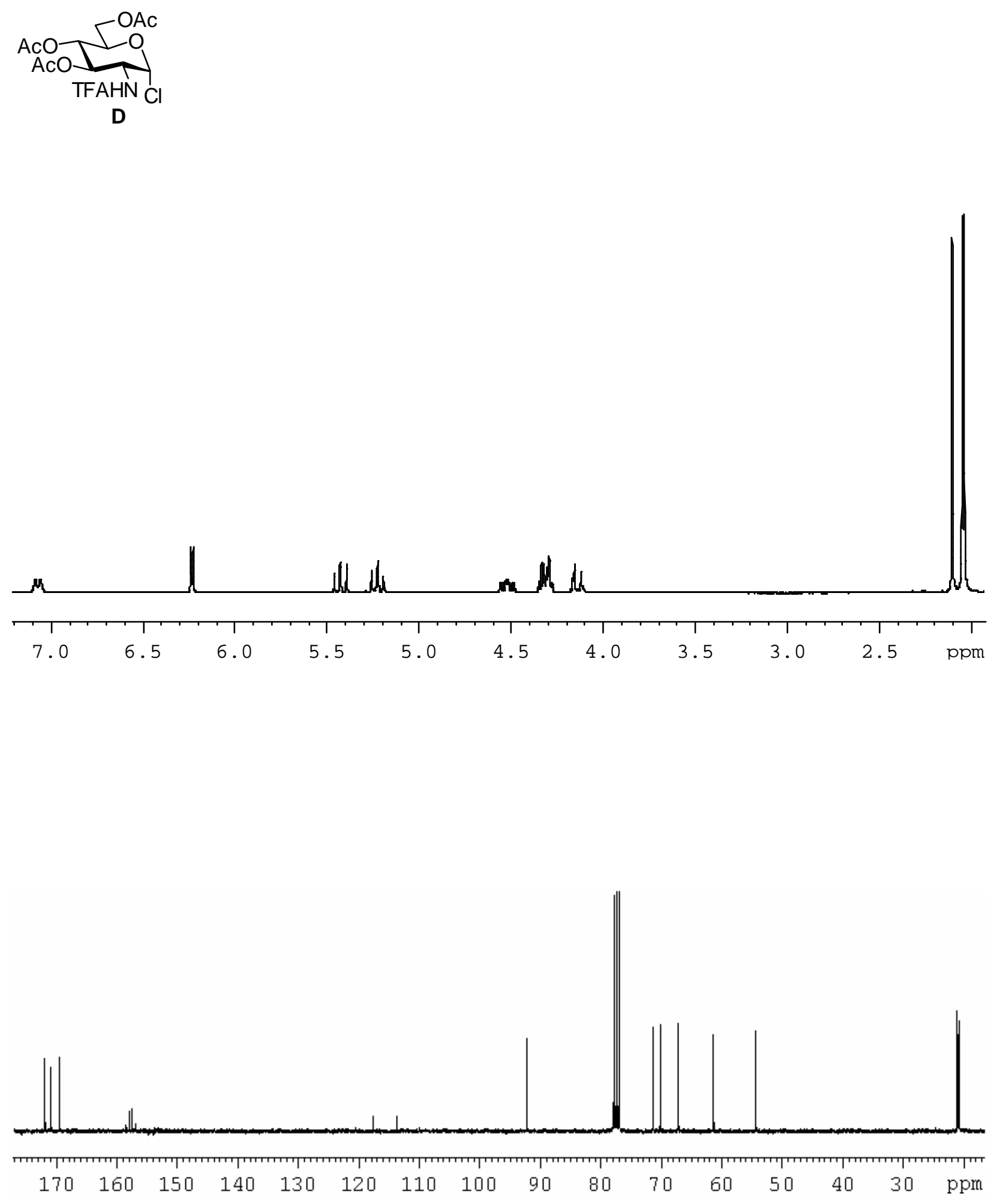


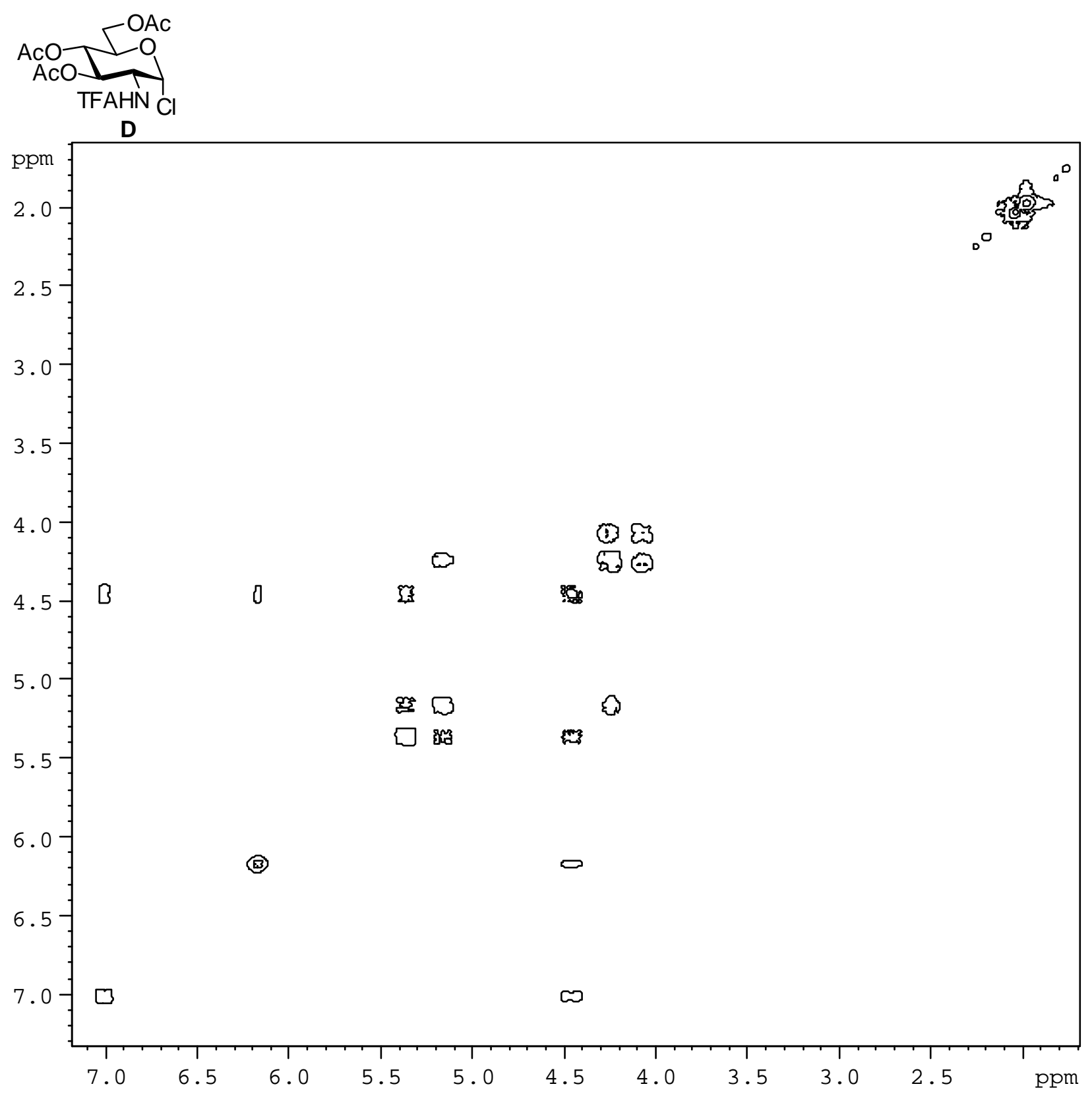



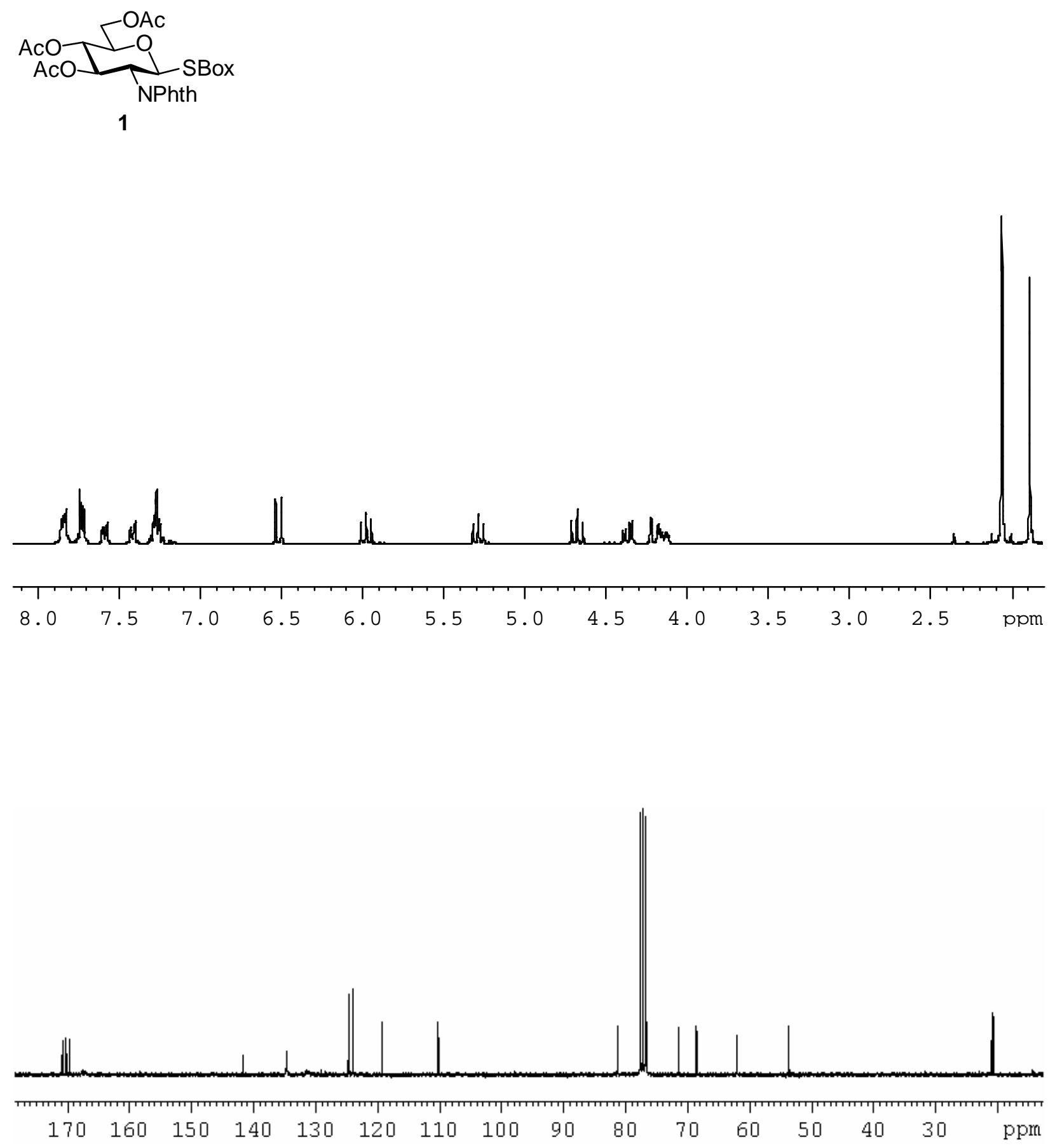


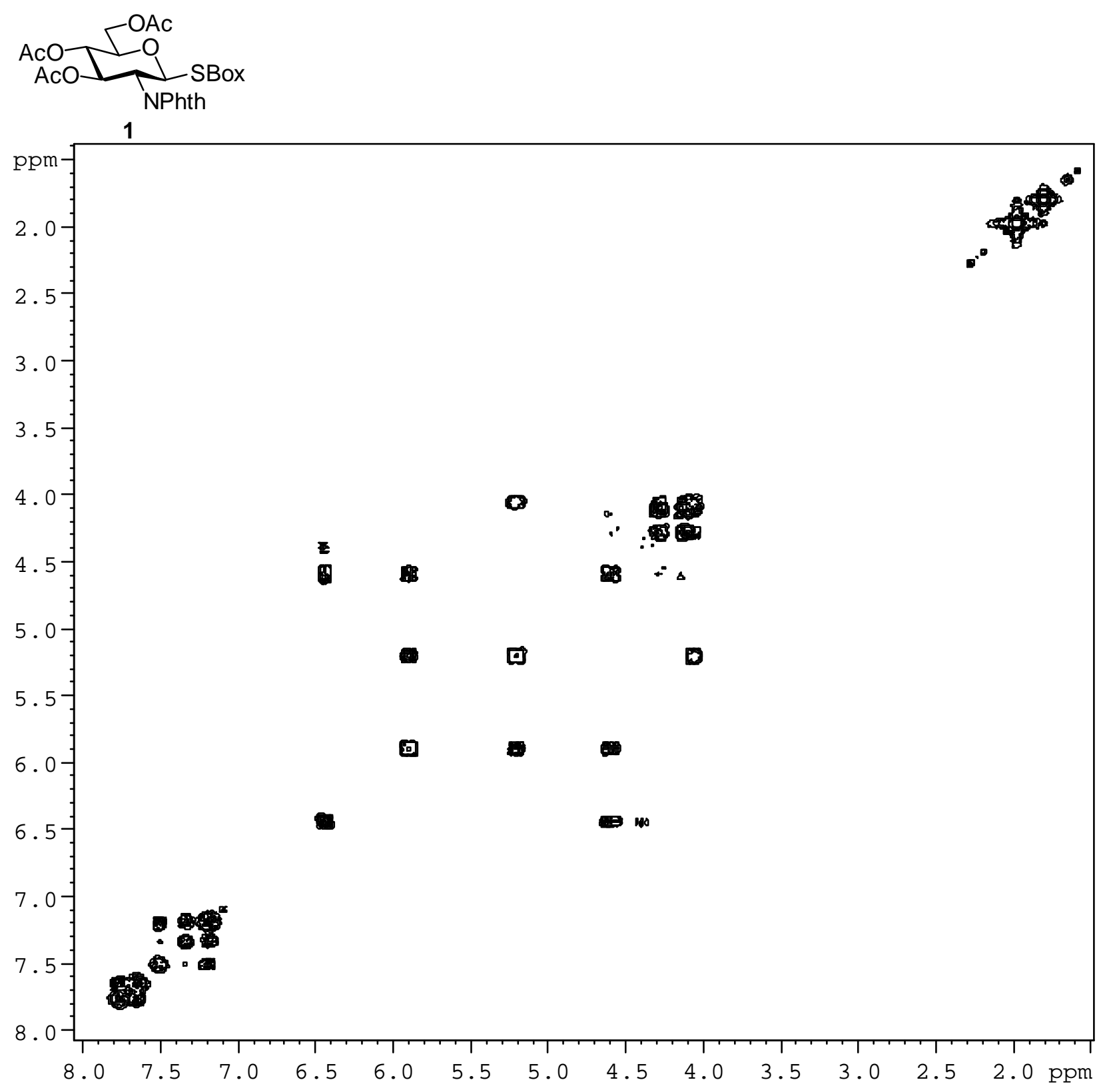



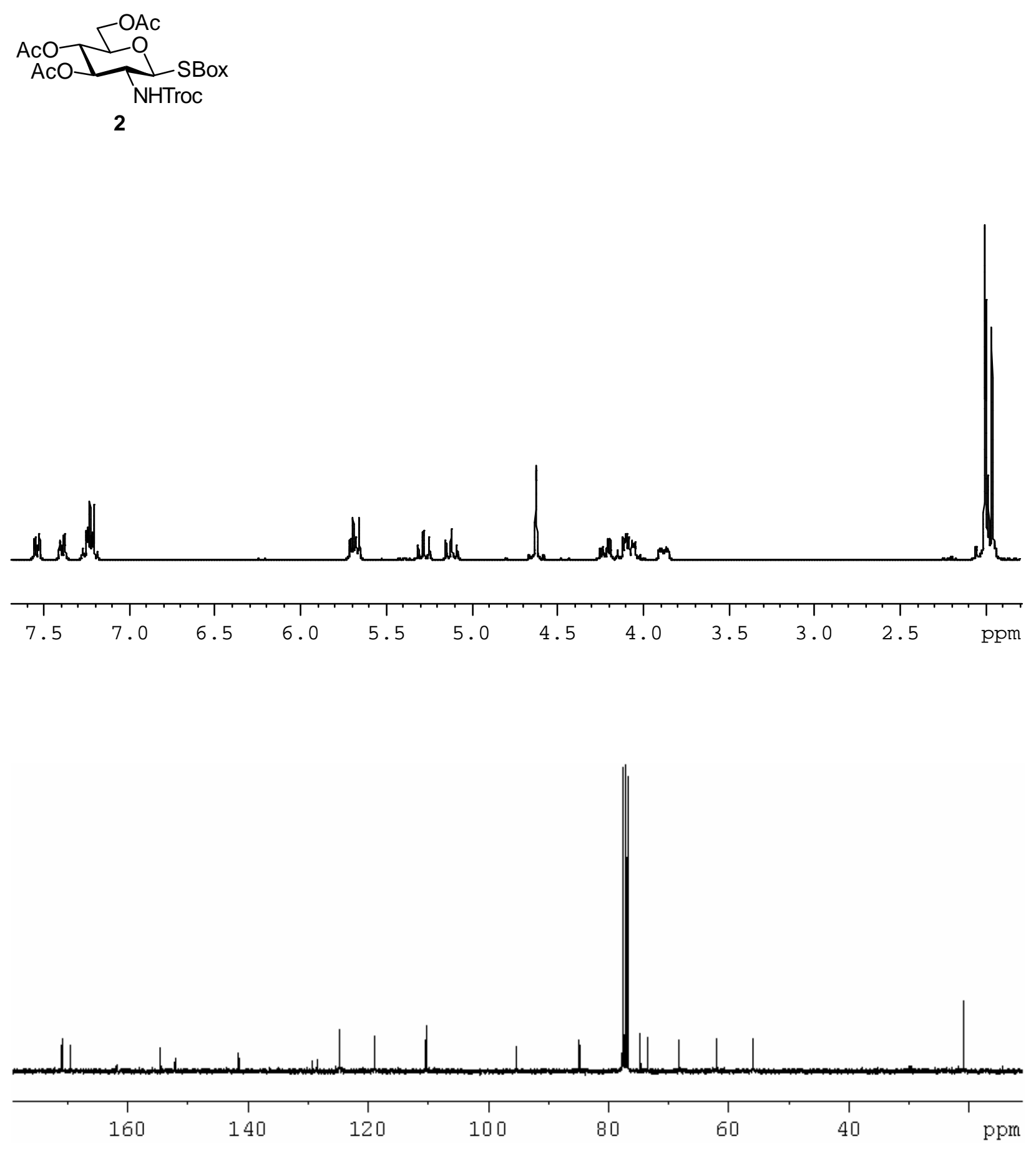


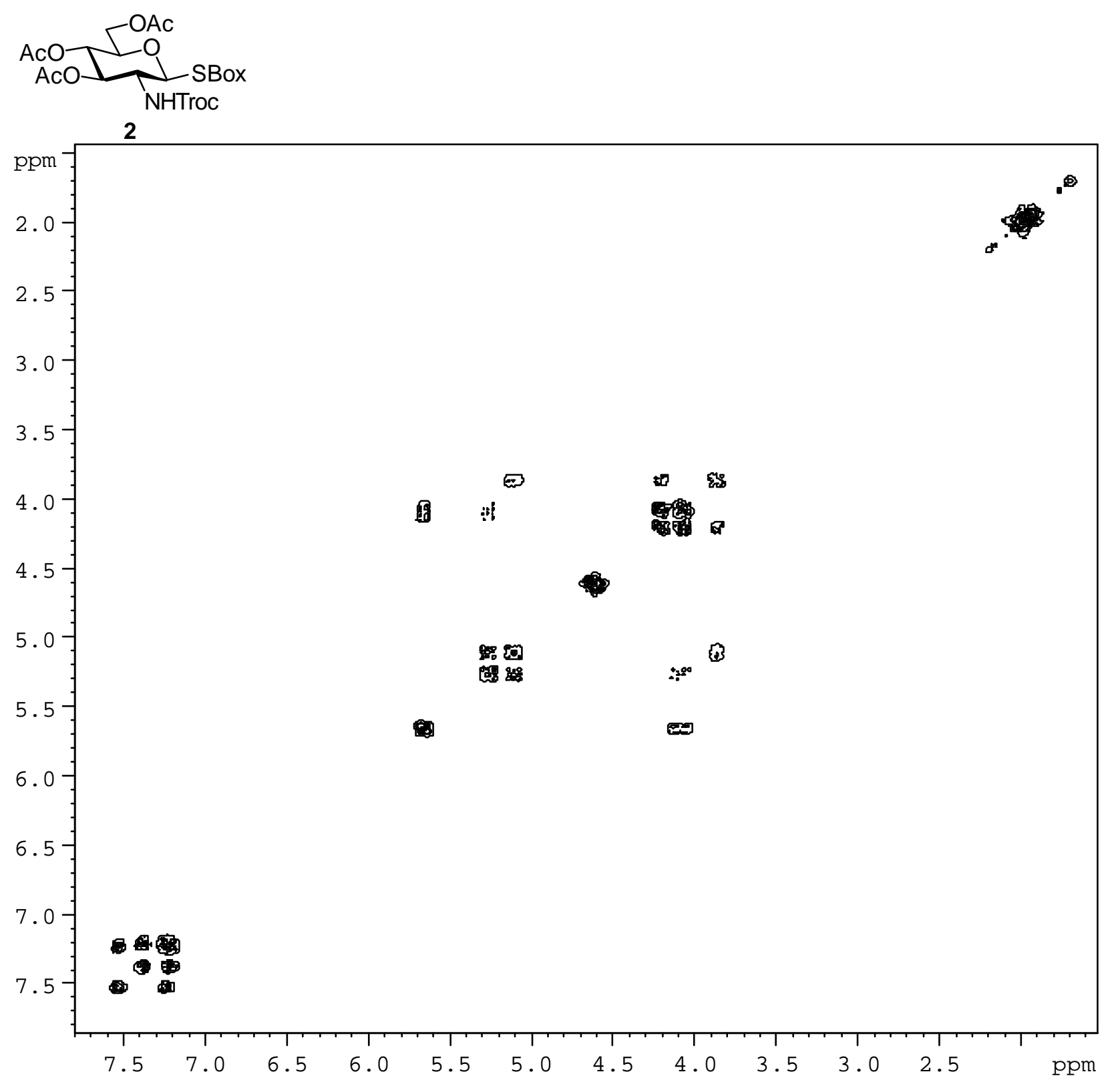



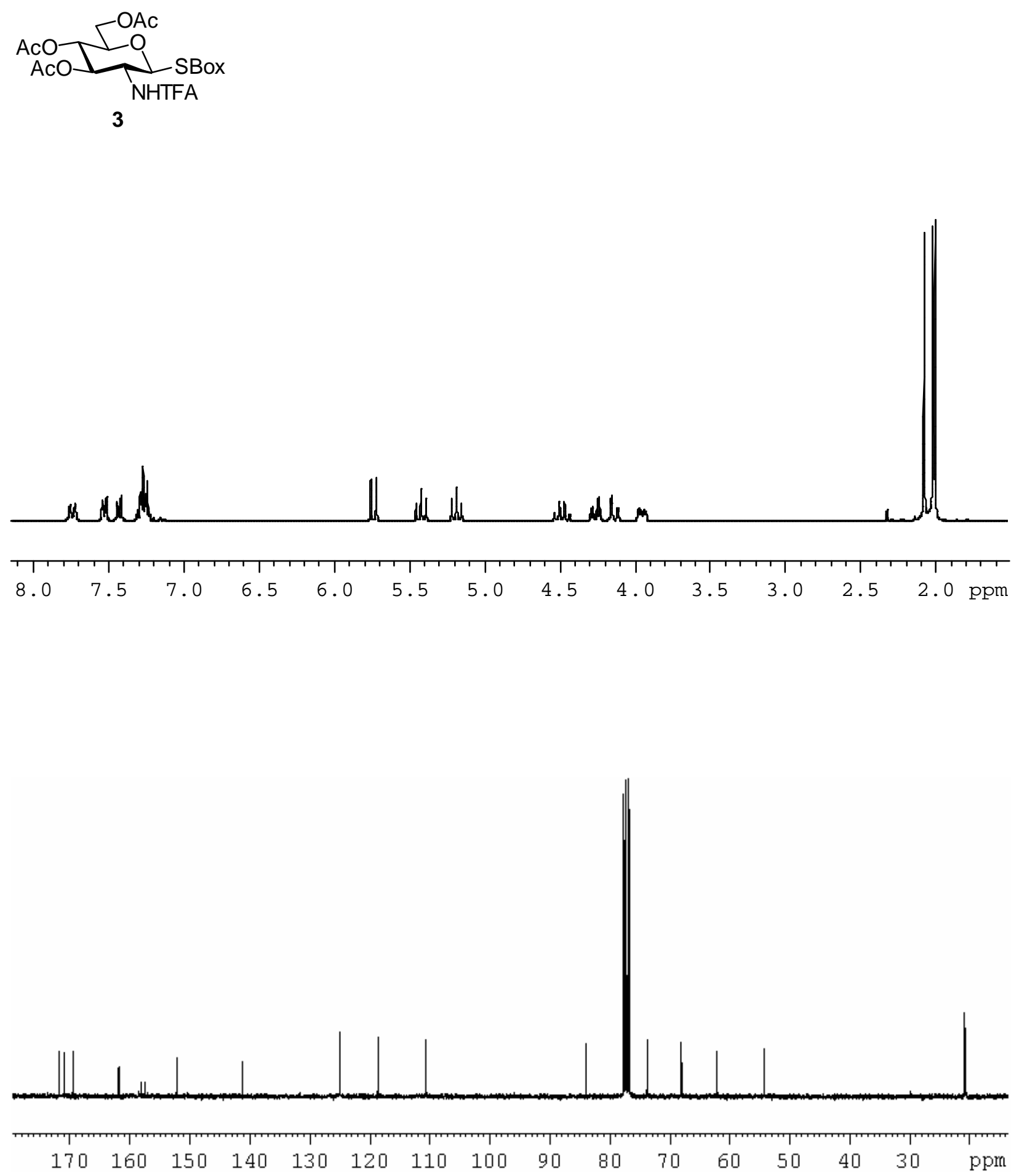


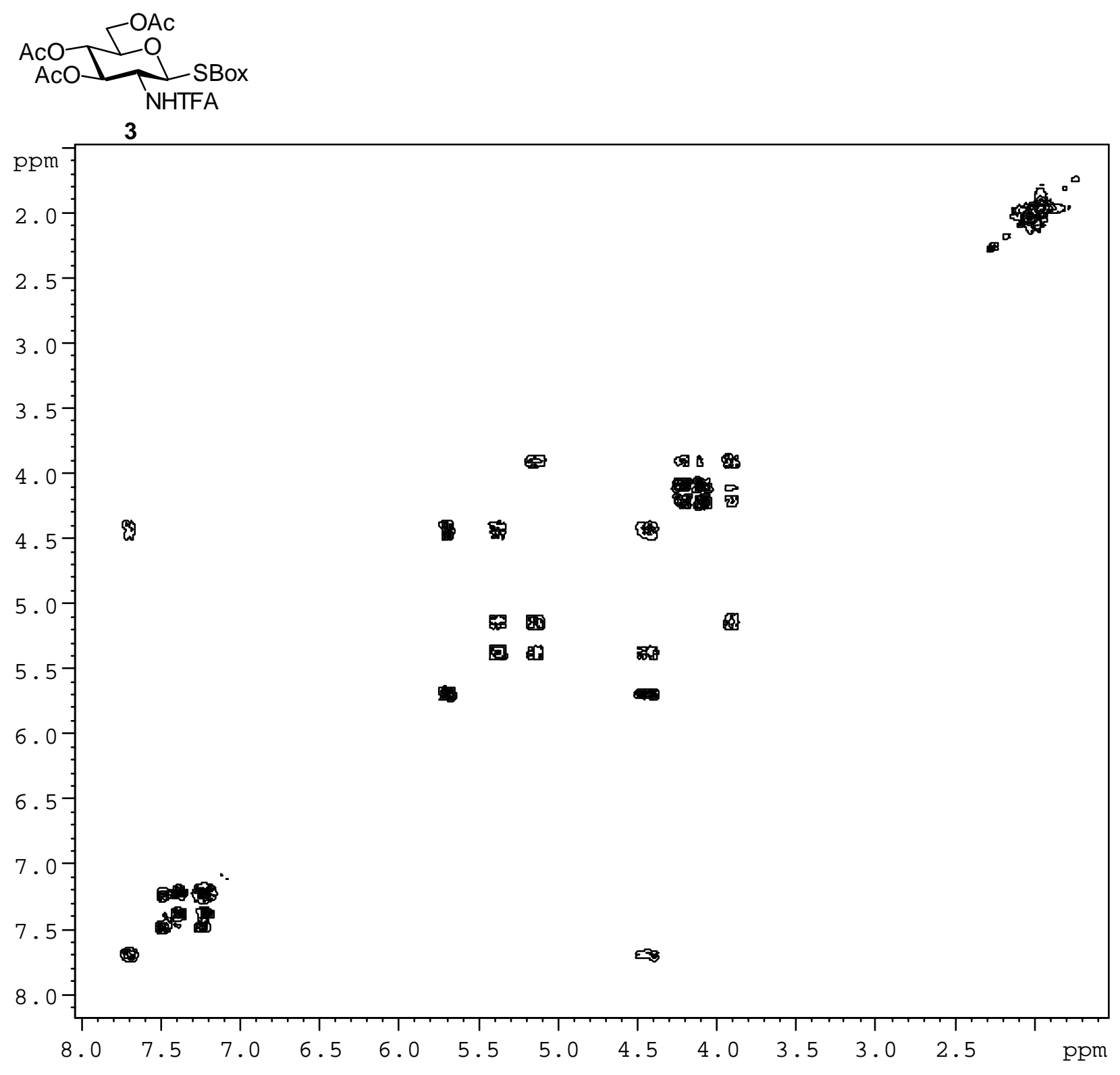




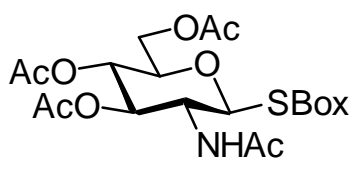

4

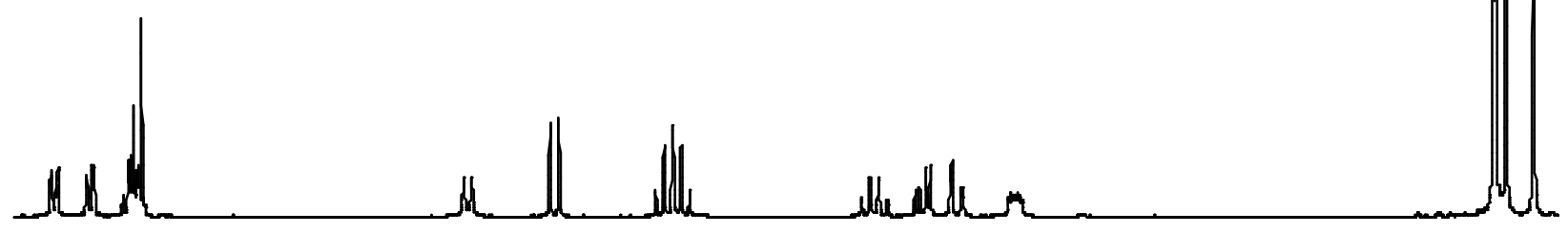

$\begin{array}{llllllllllll}7.5 & 7.0 & 6.5 & 6.0 & 5.5 & 5.0 & 4.5 & 4.0 & 3.5 & 3.0 & 2.5 & \text { ppm }\end{array}$

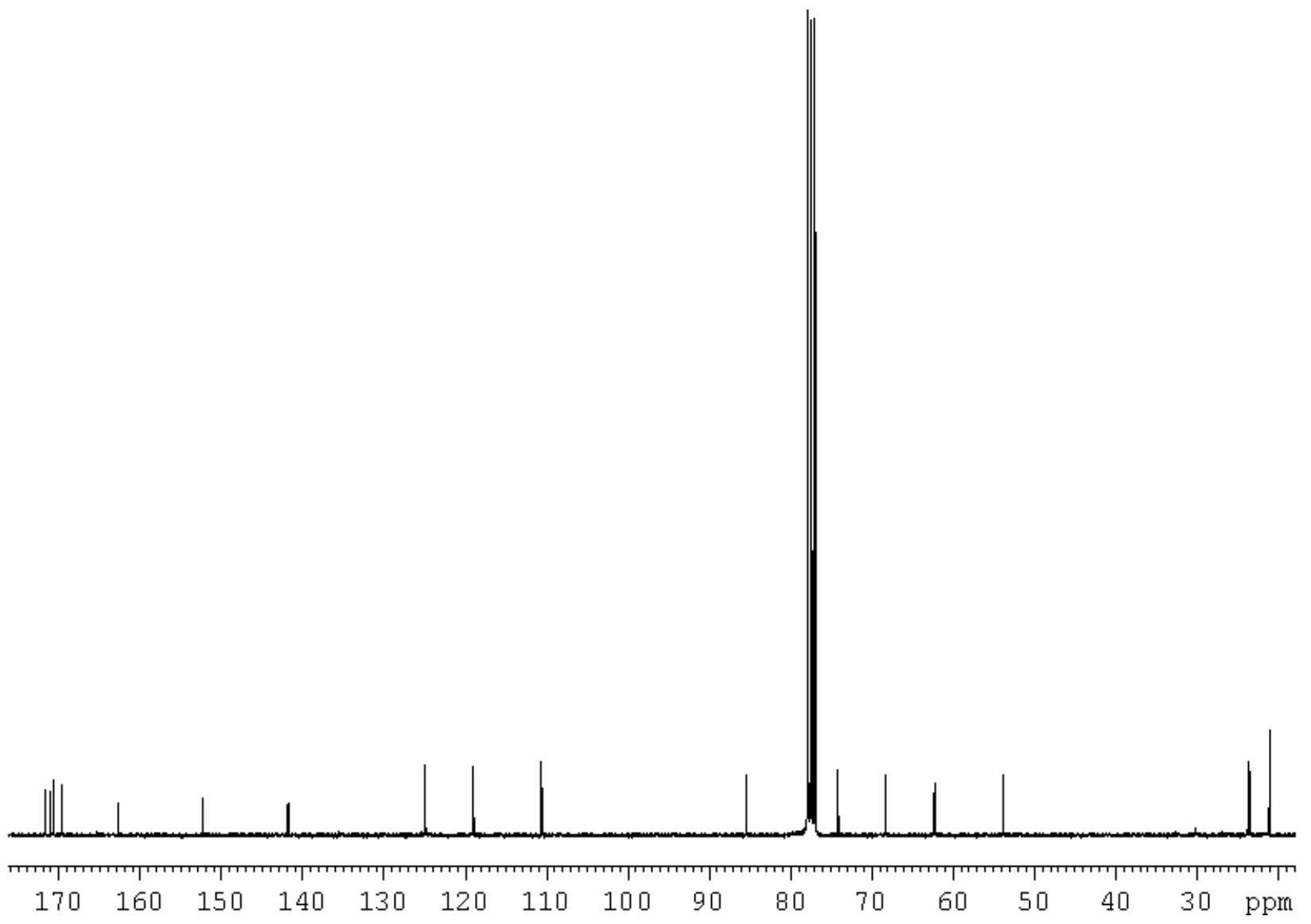

S-23 


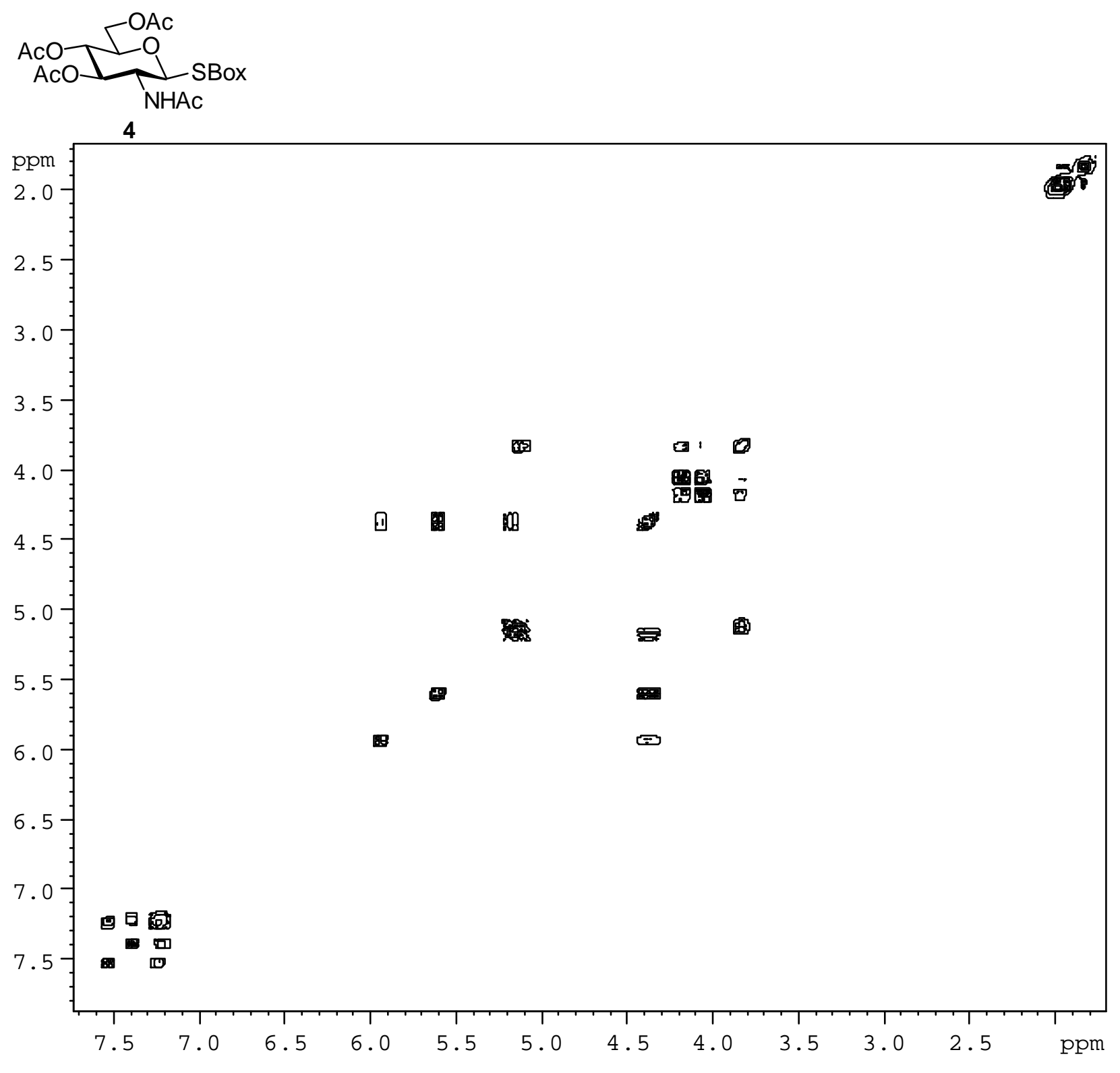



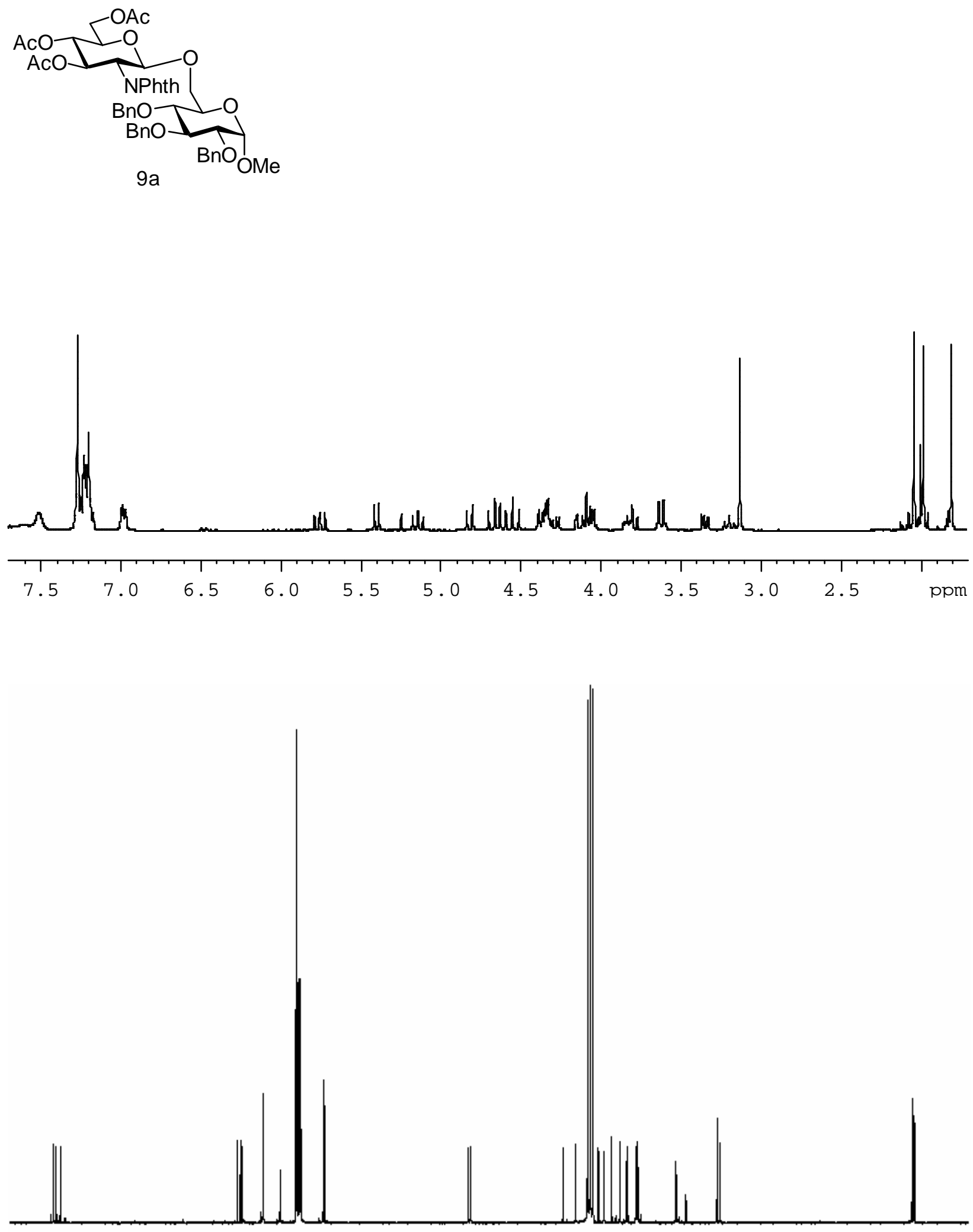

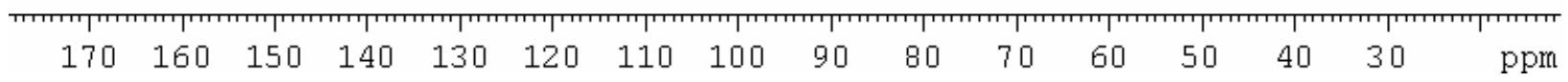

S-25 


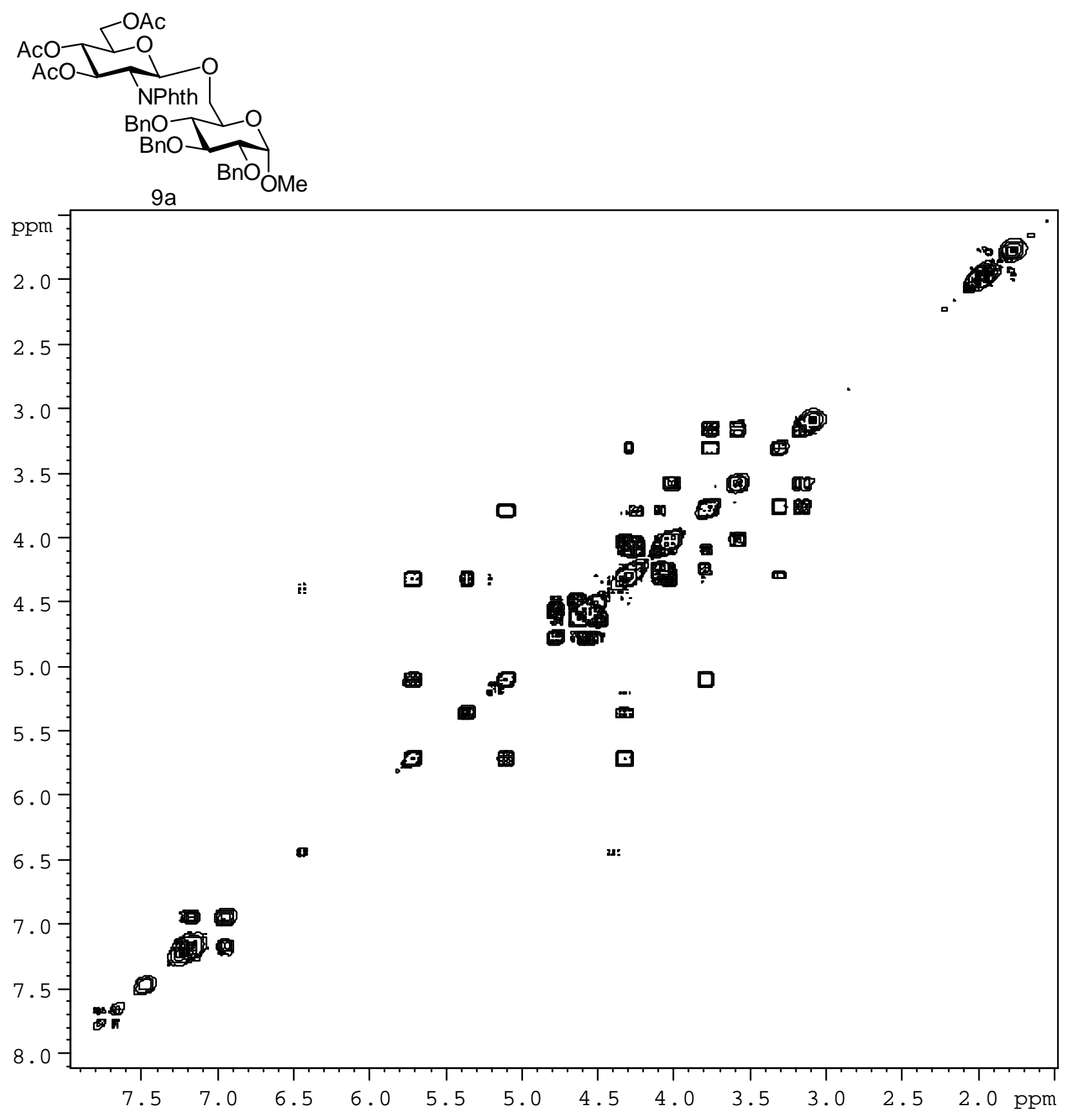



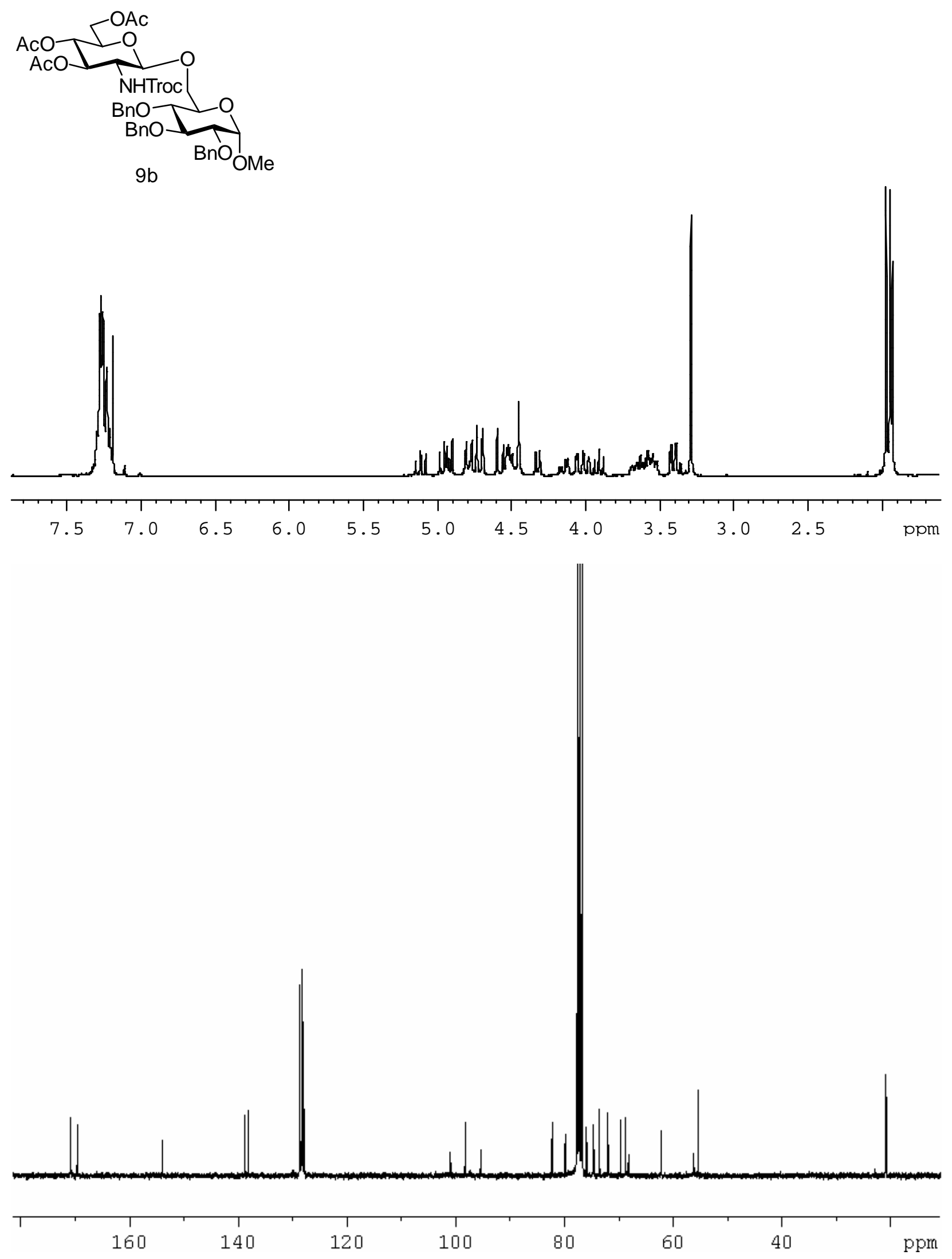


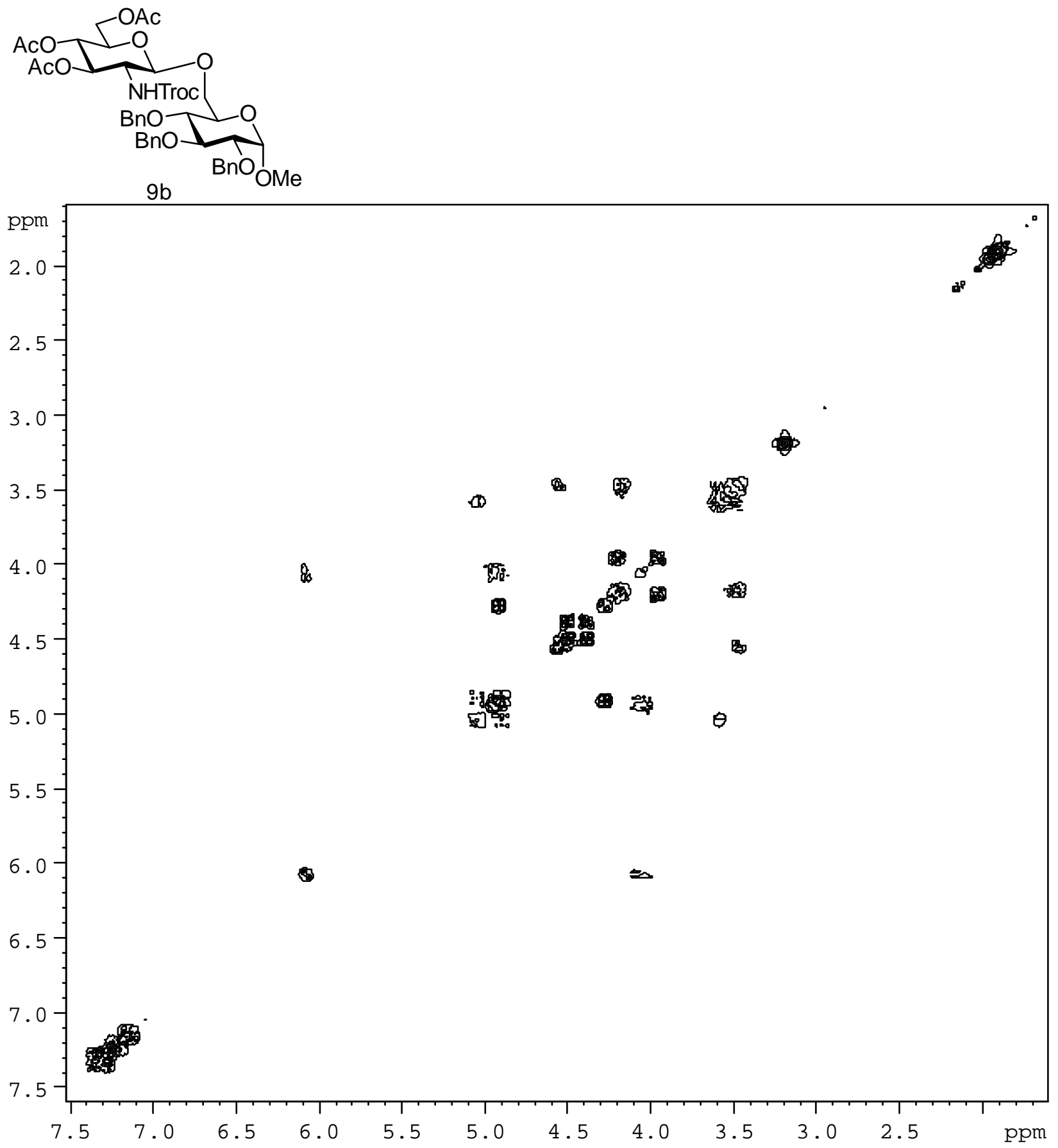

S-28 

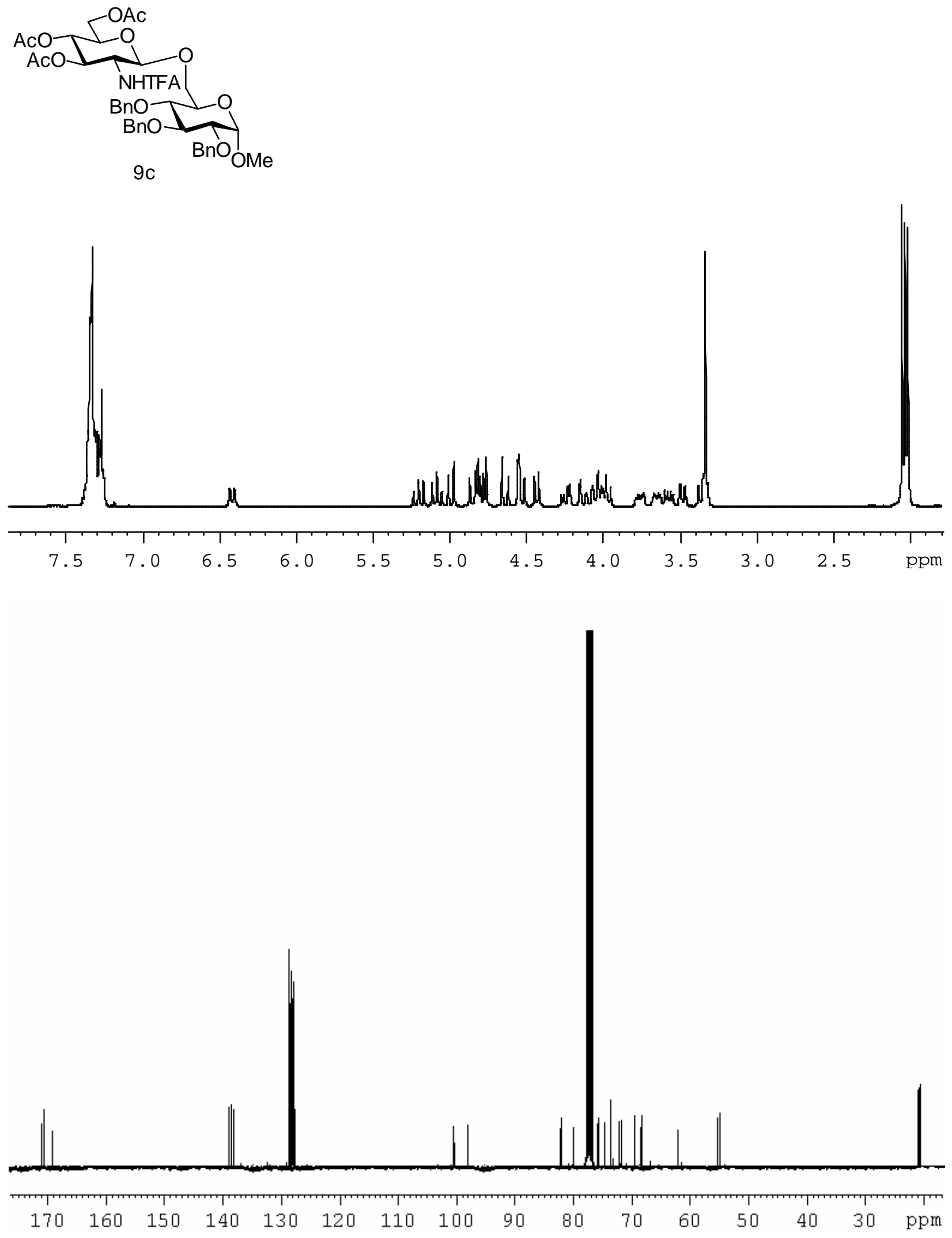

S-29 


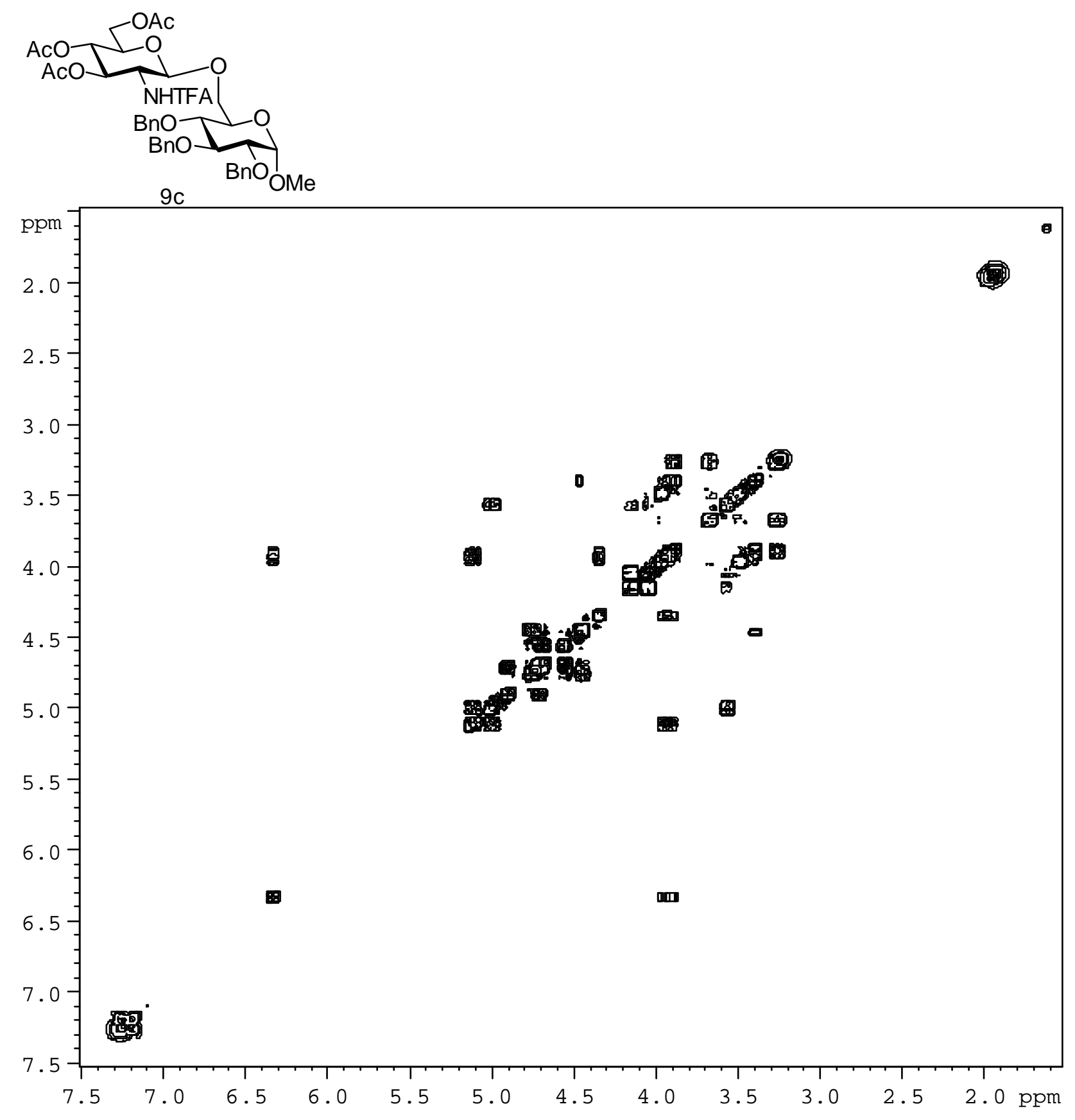



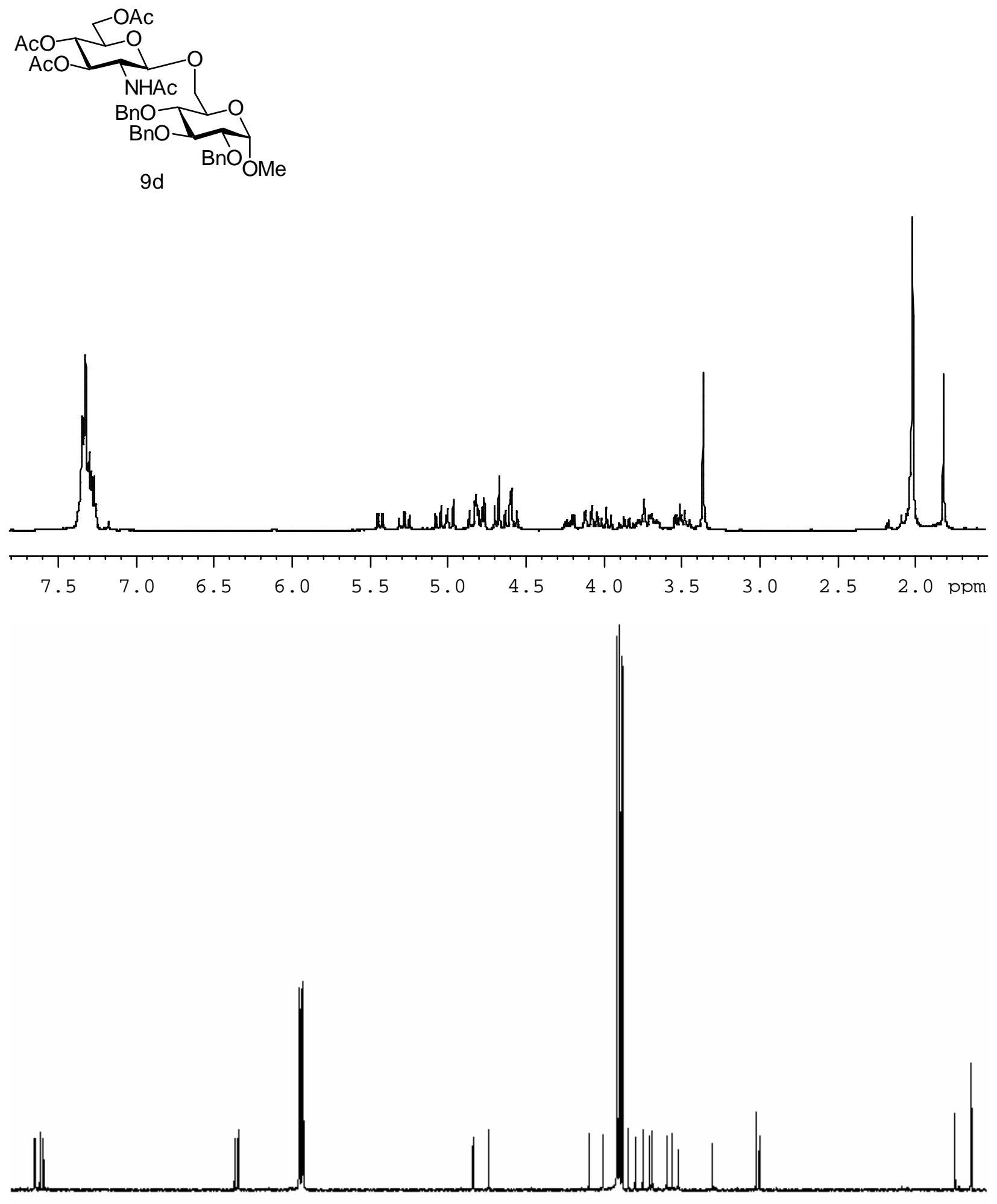

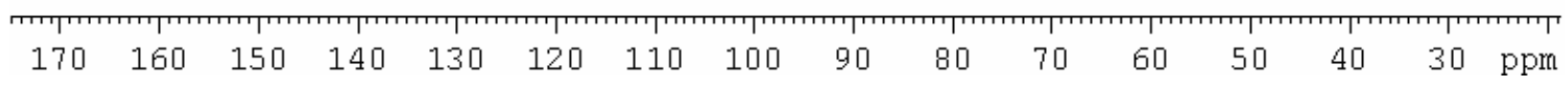

S-31 


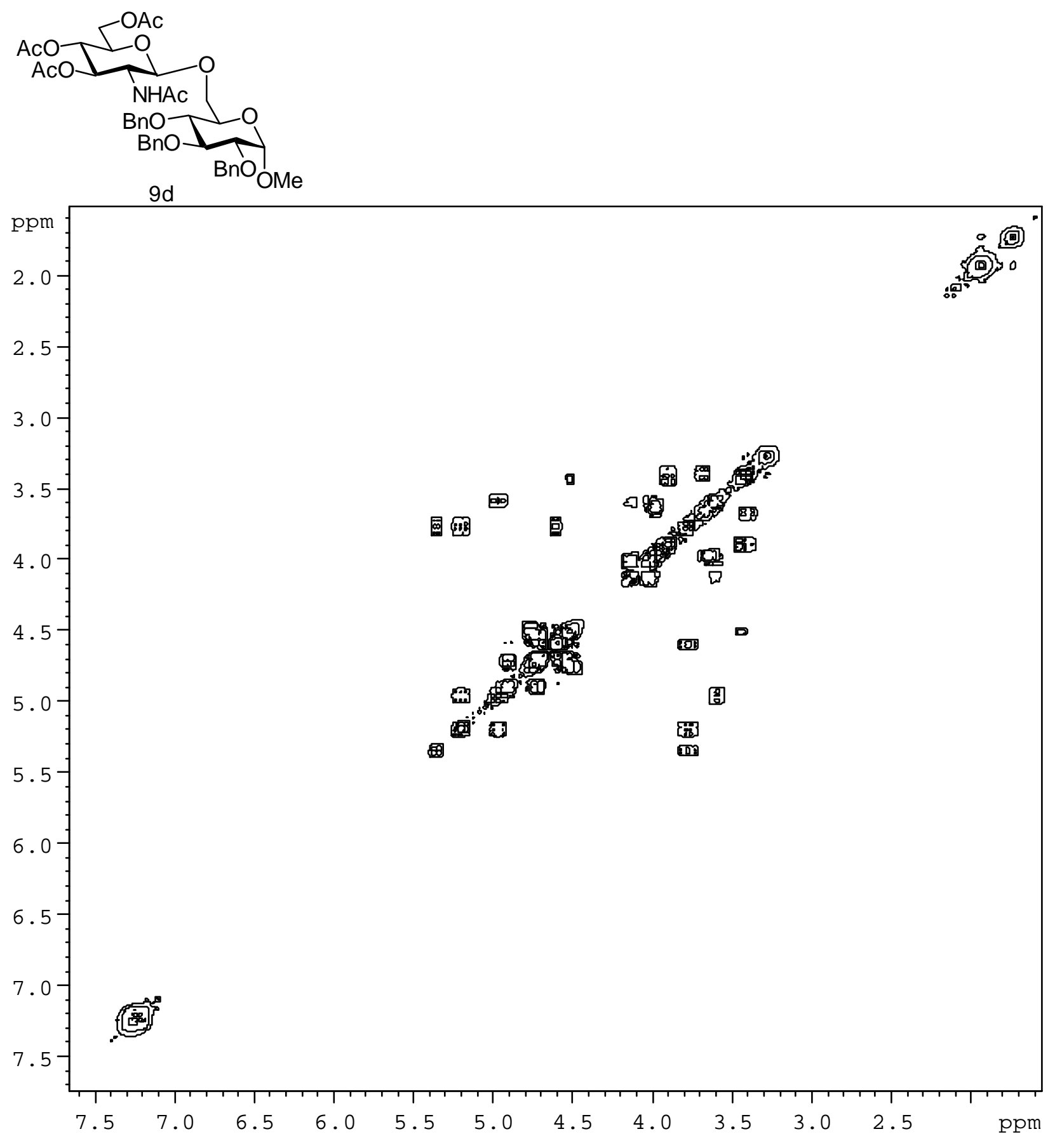



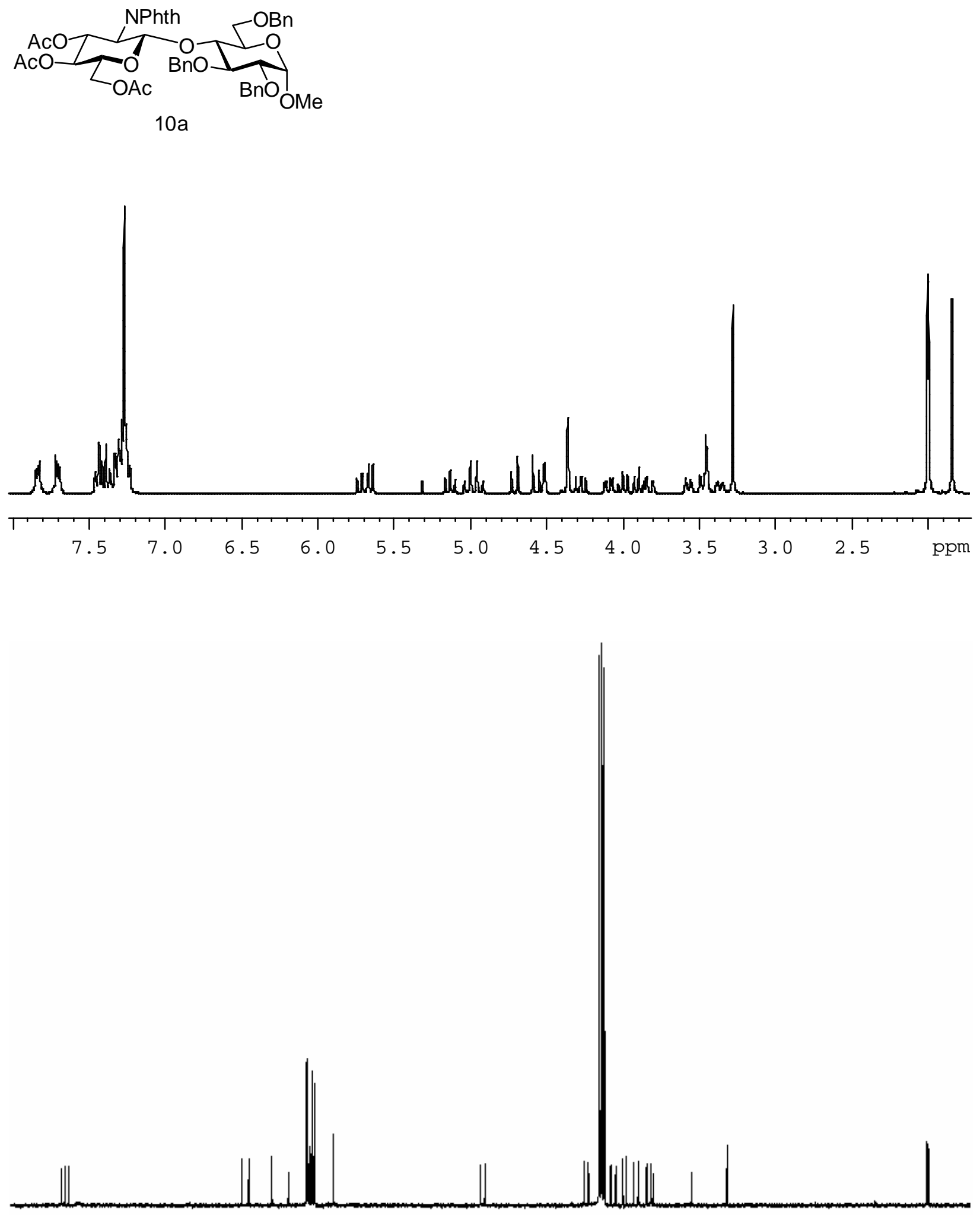

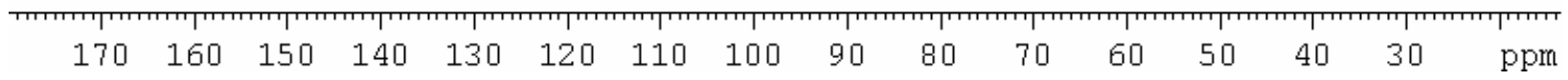

S-33 


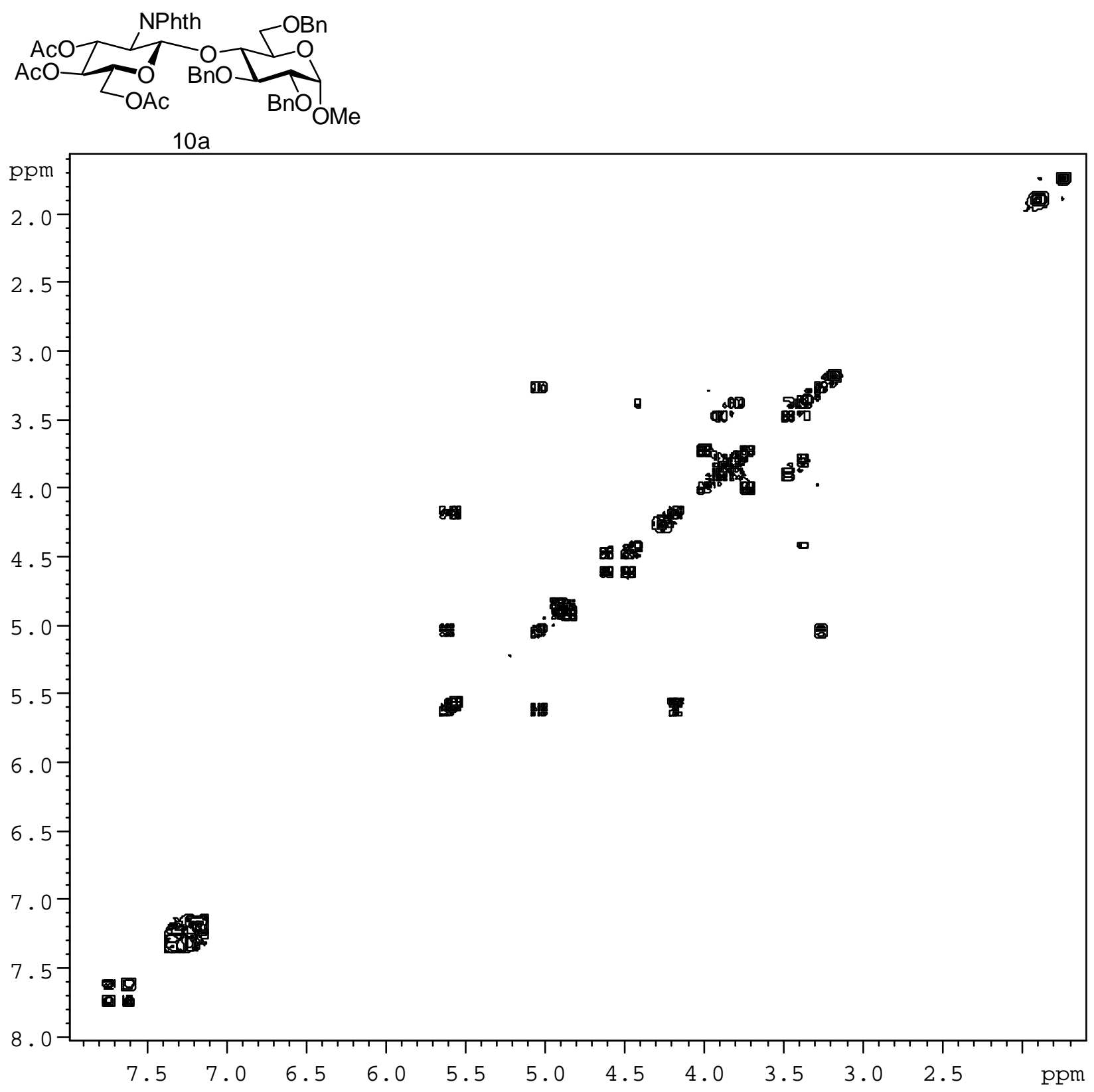



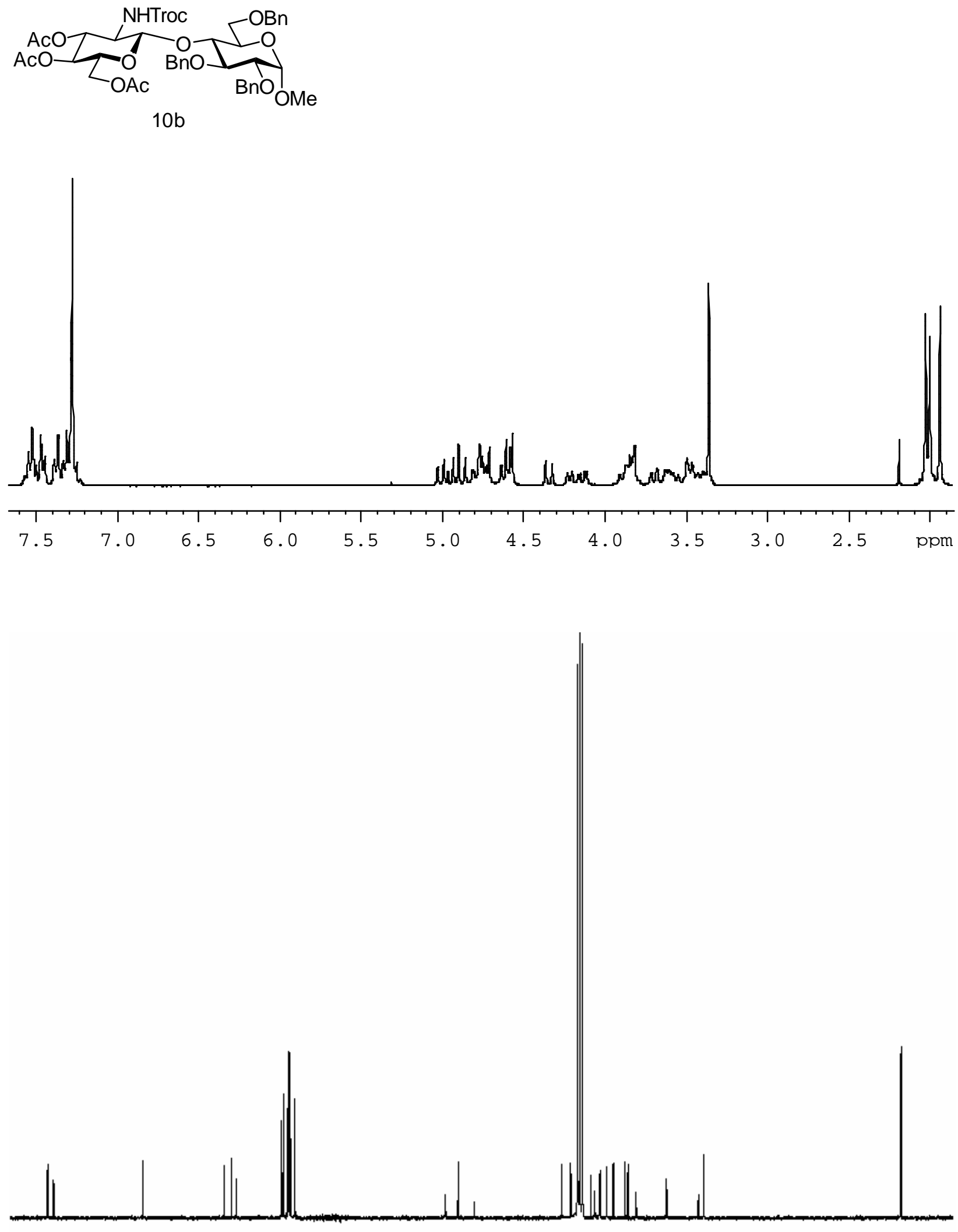

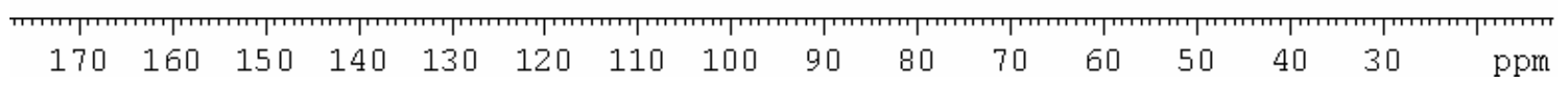

S-35 

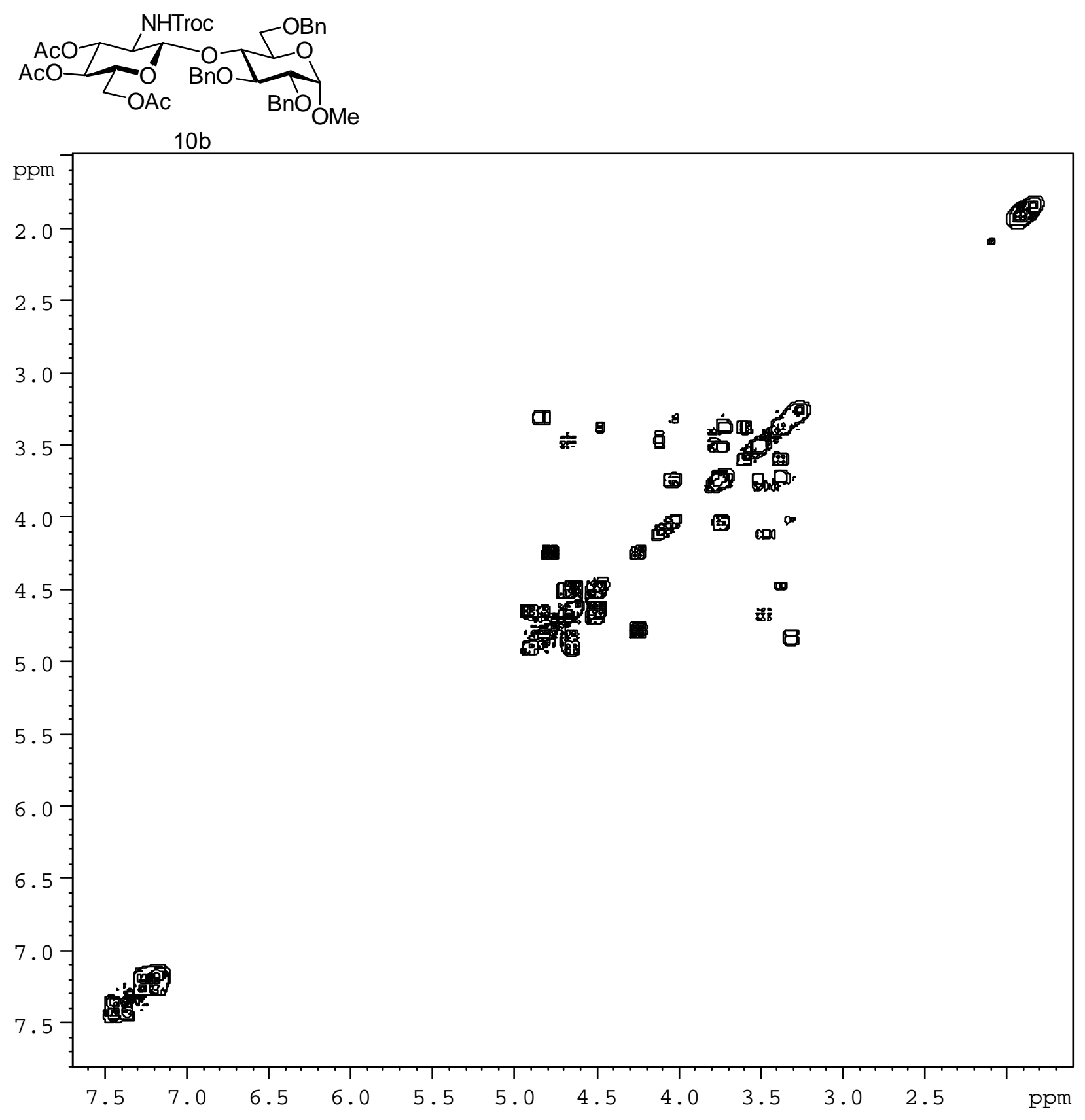

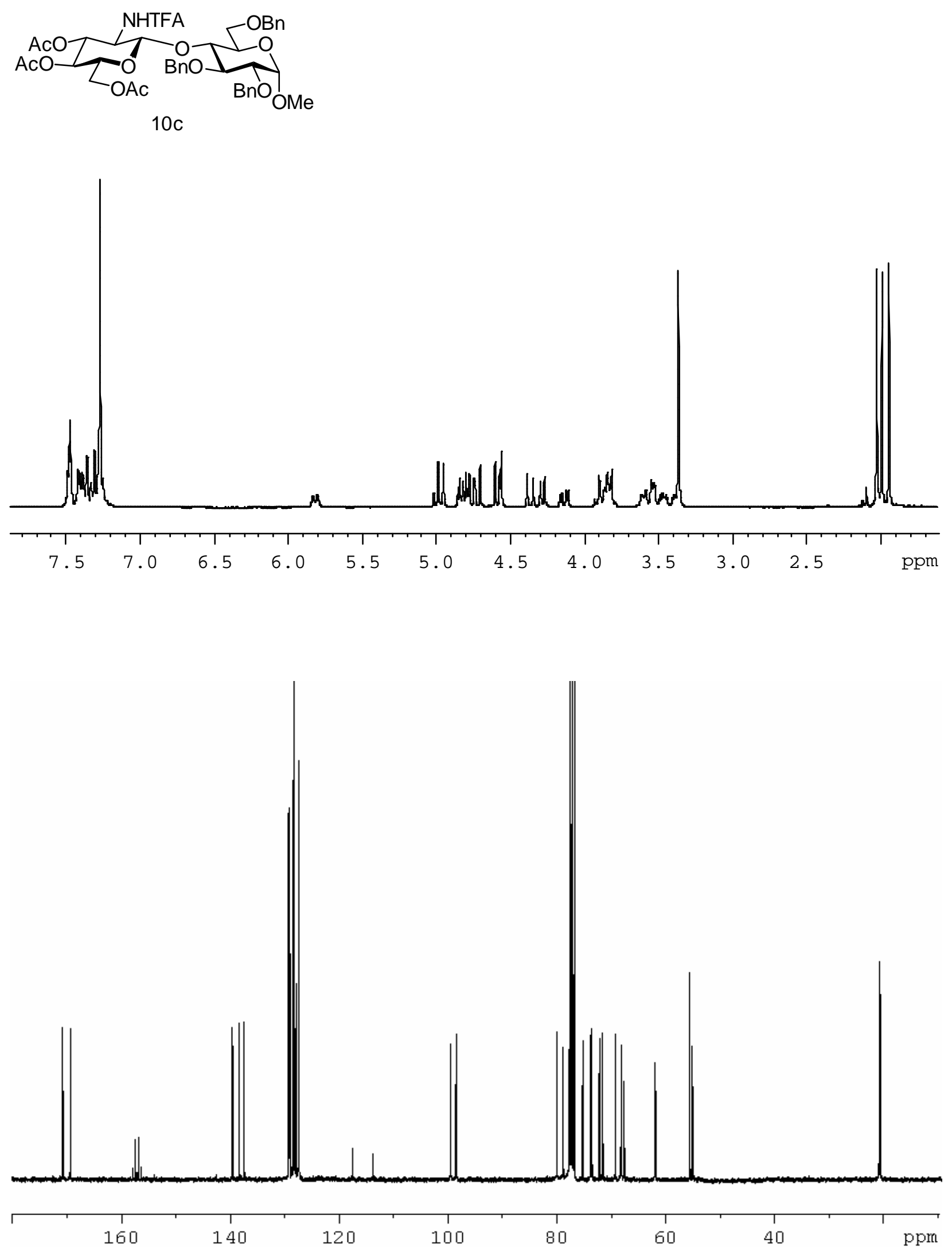

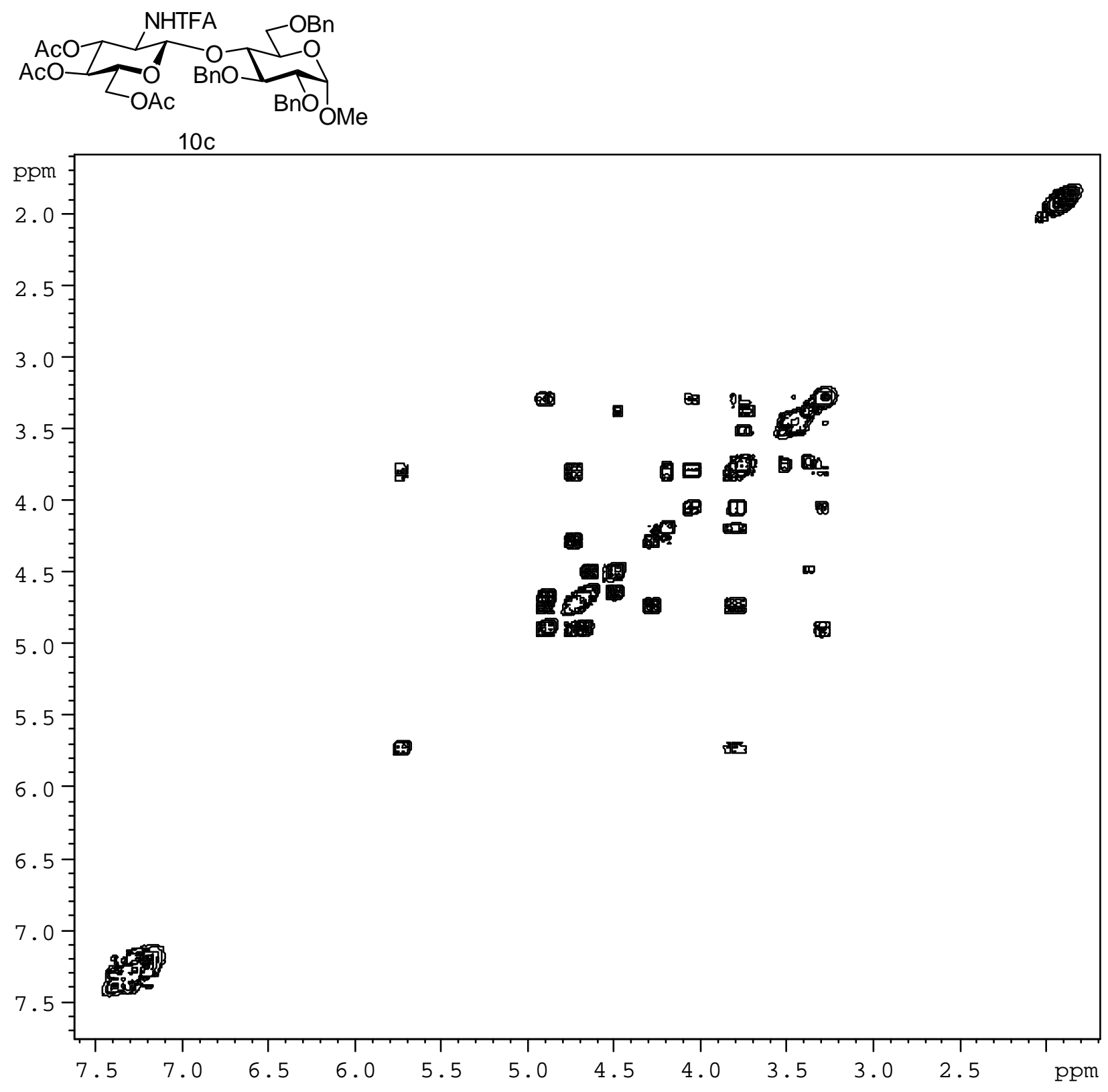

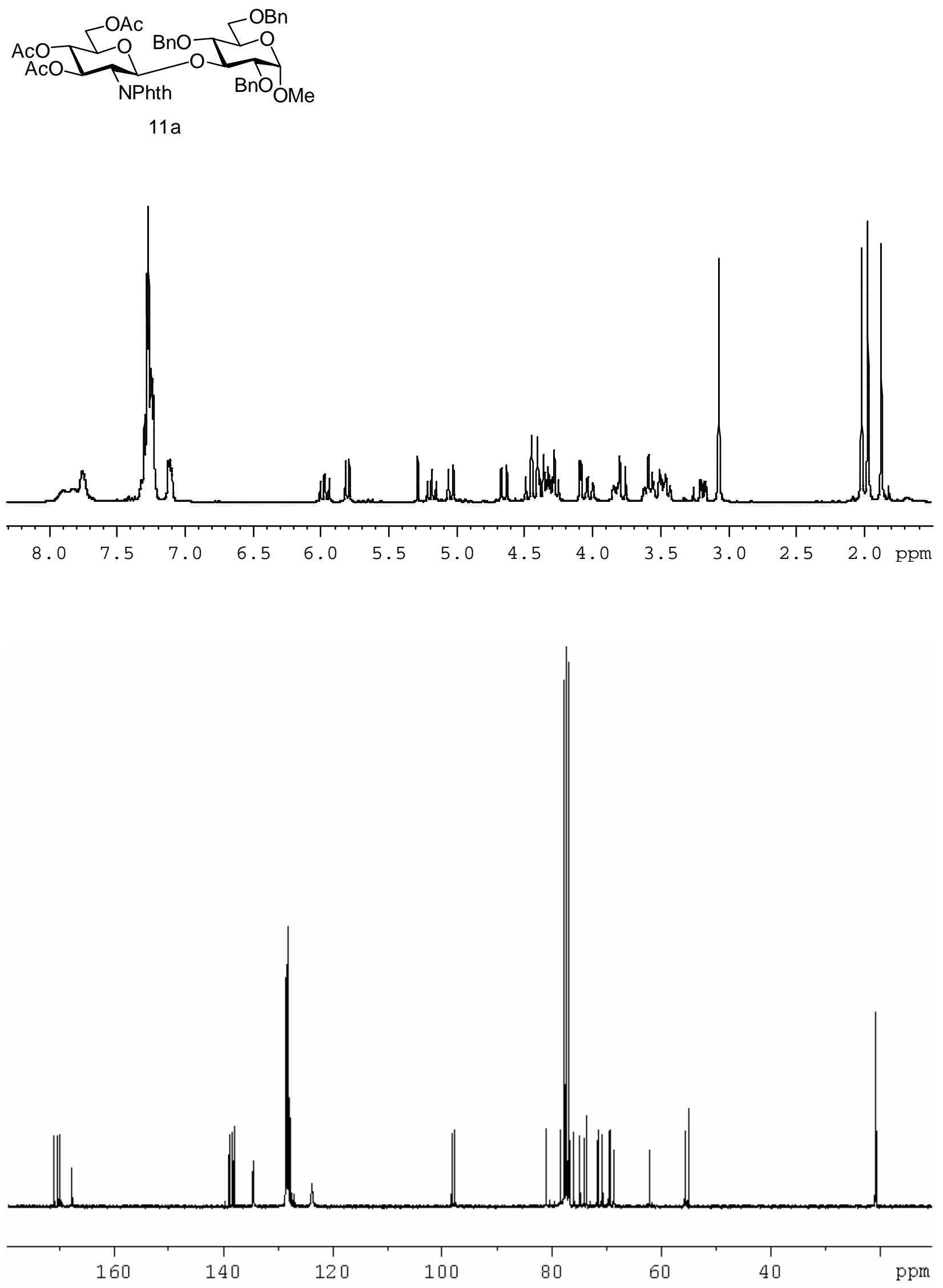

S-39 

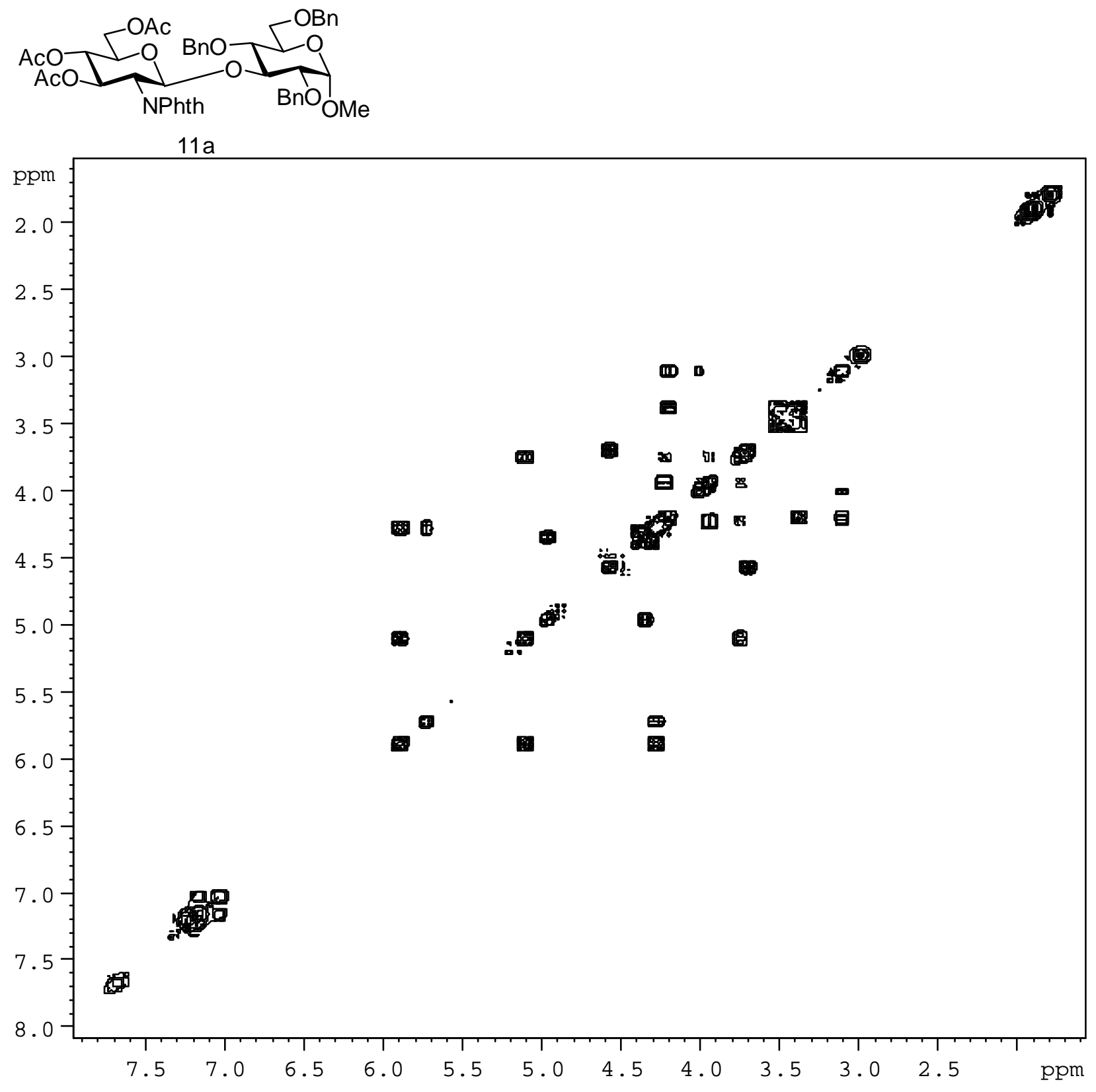

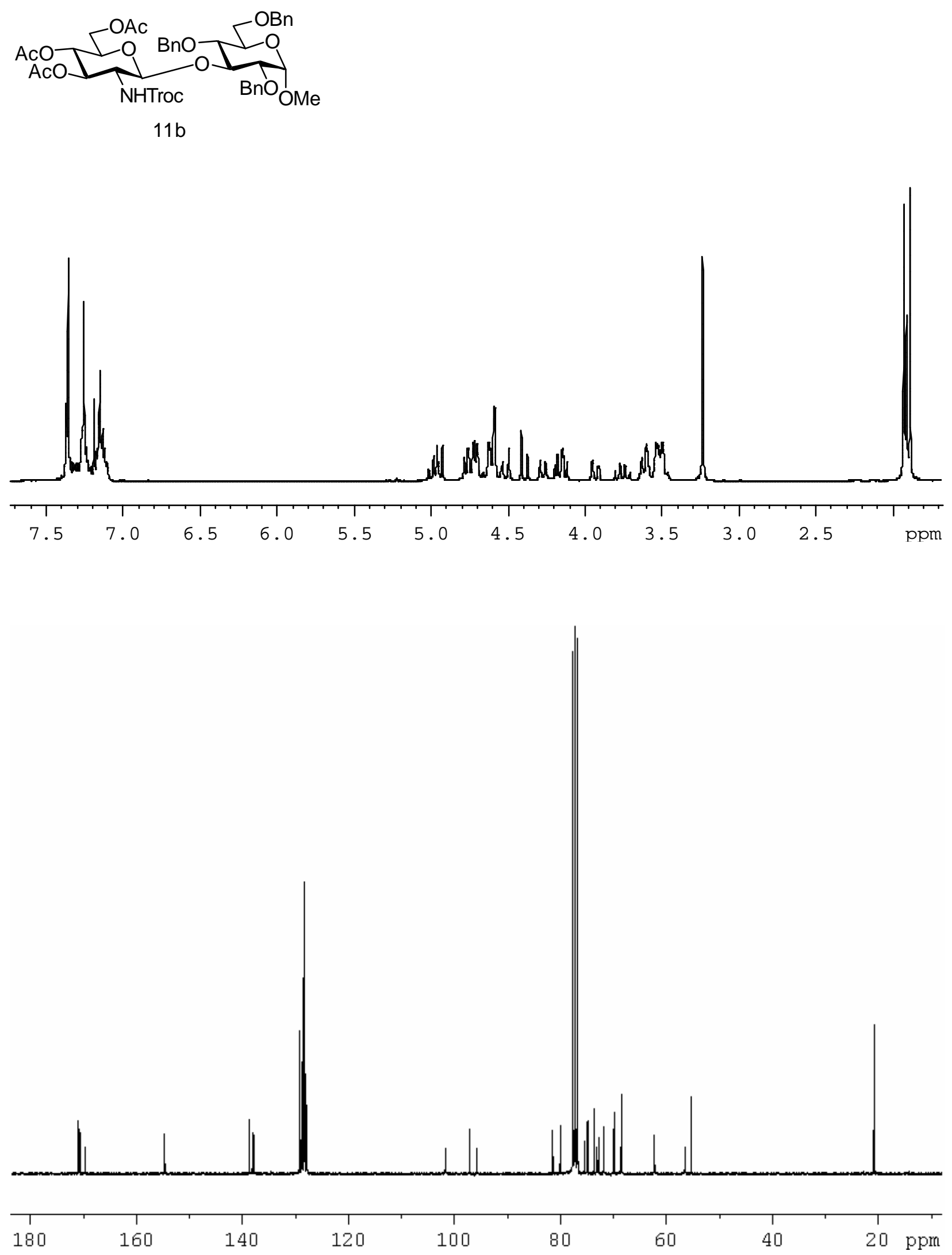

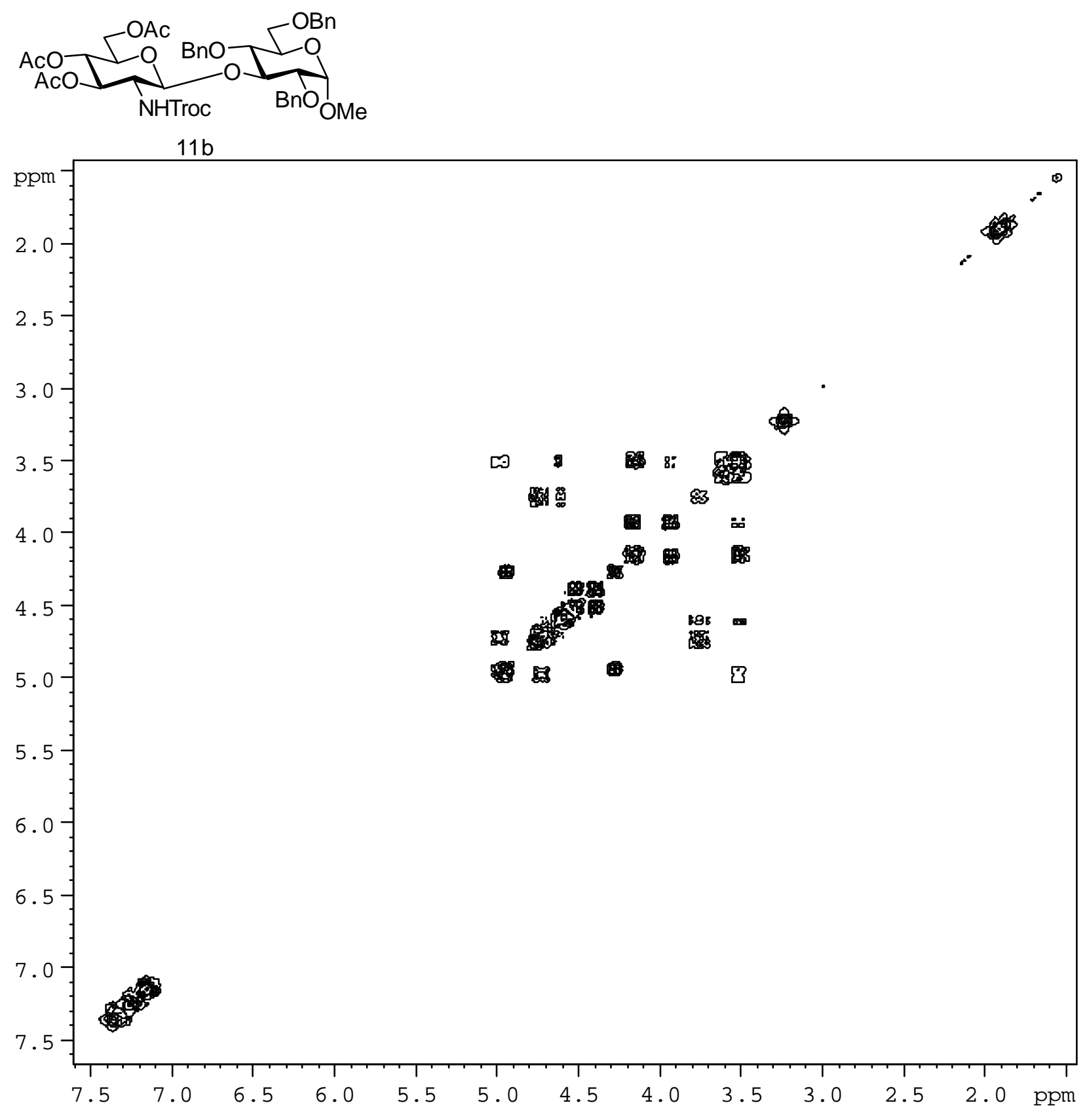


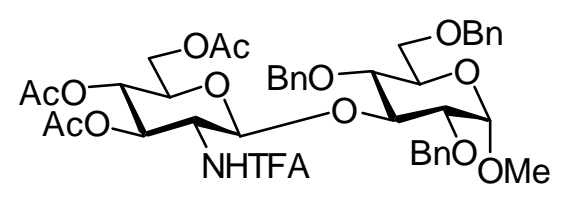

11c
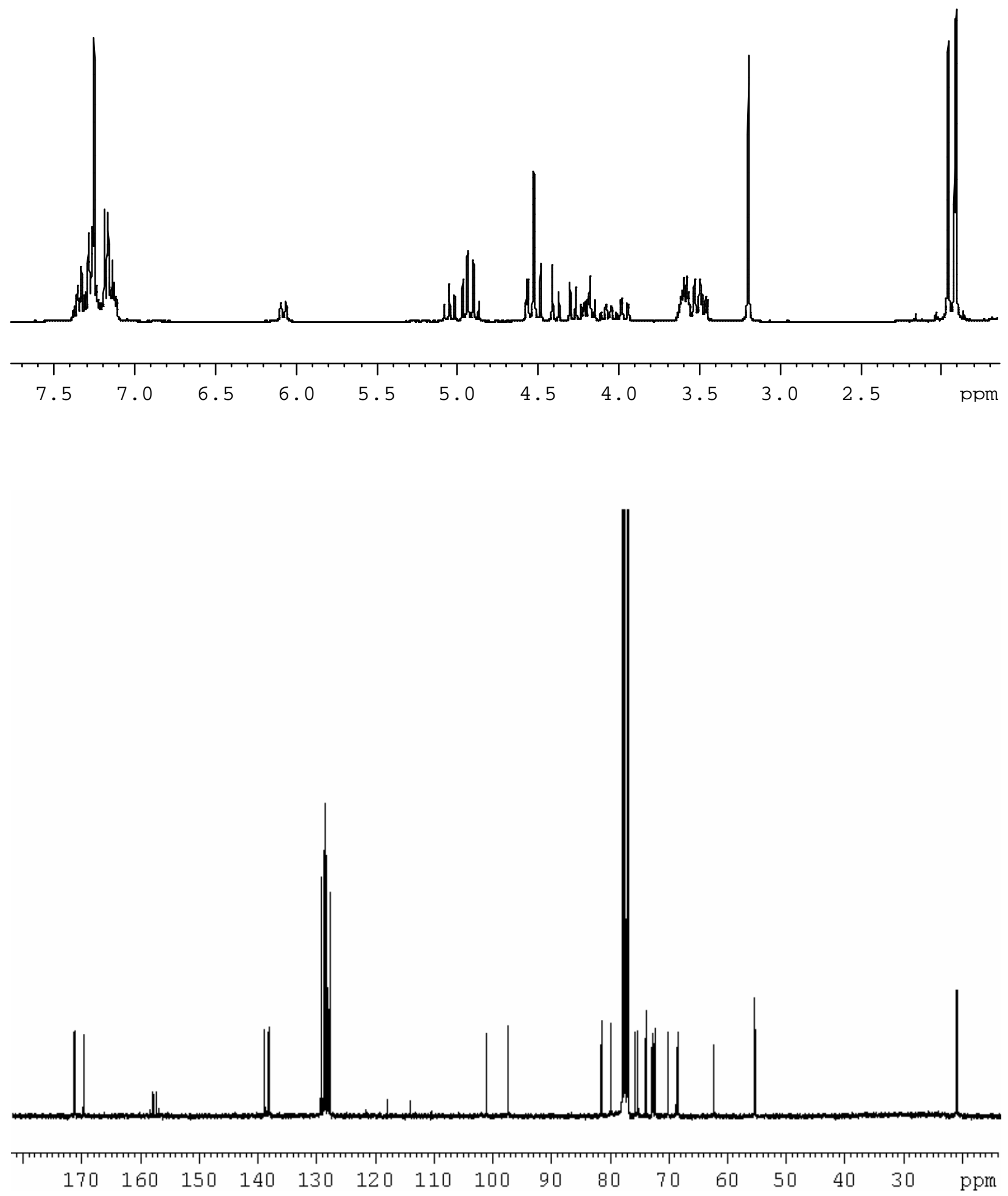


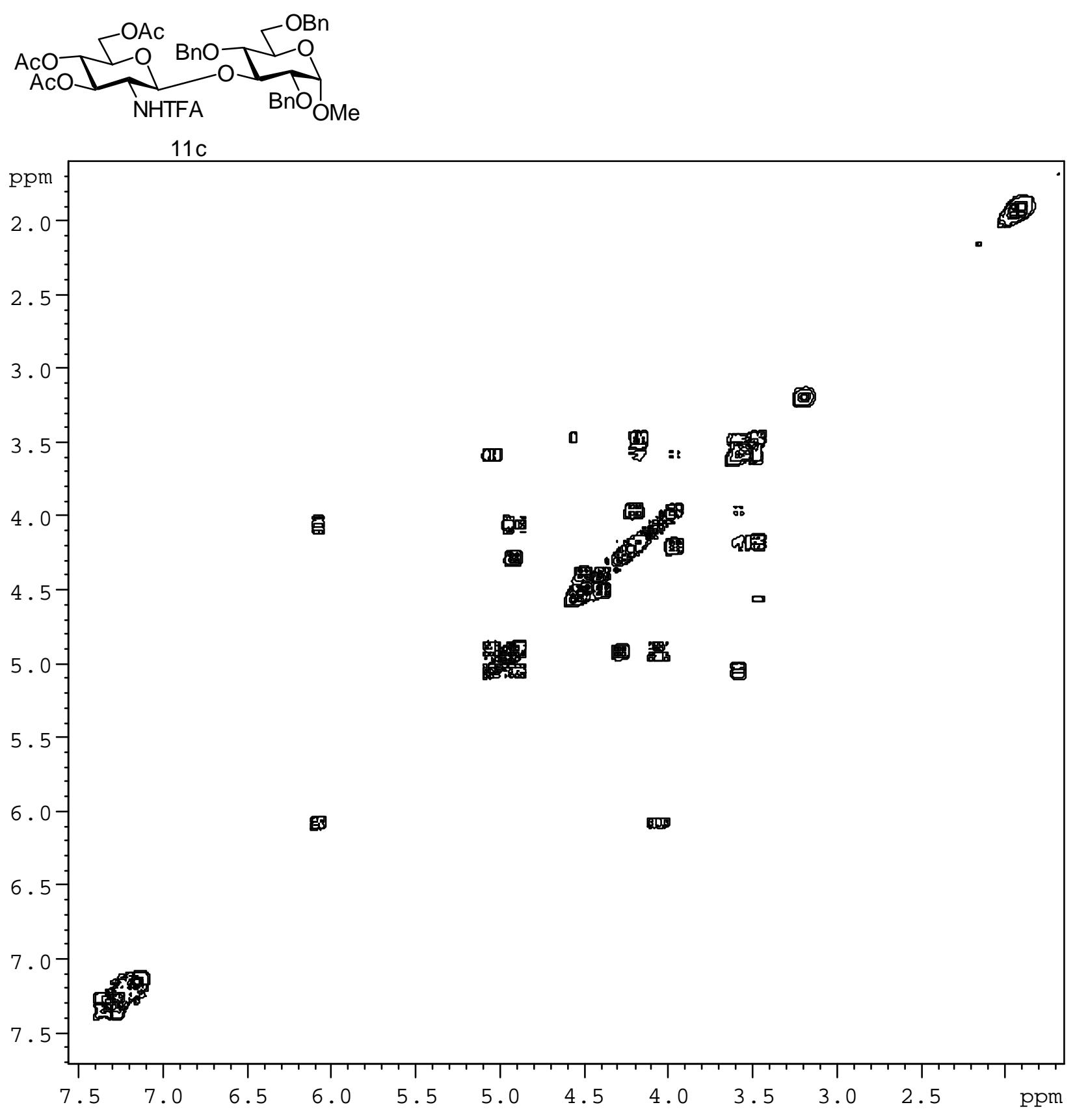




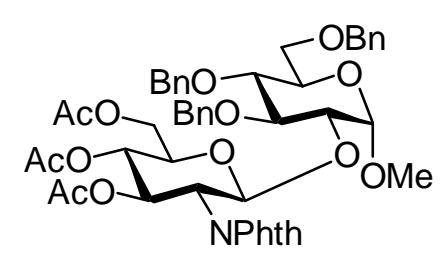

$12 \mathrm{a}$
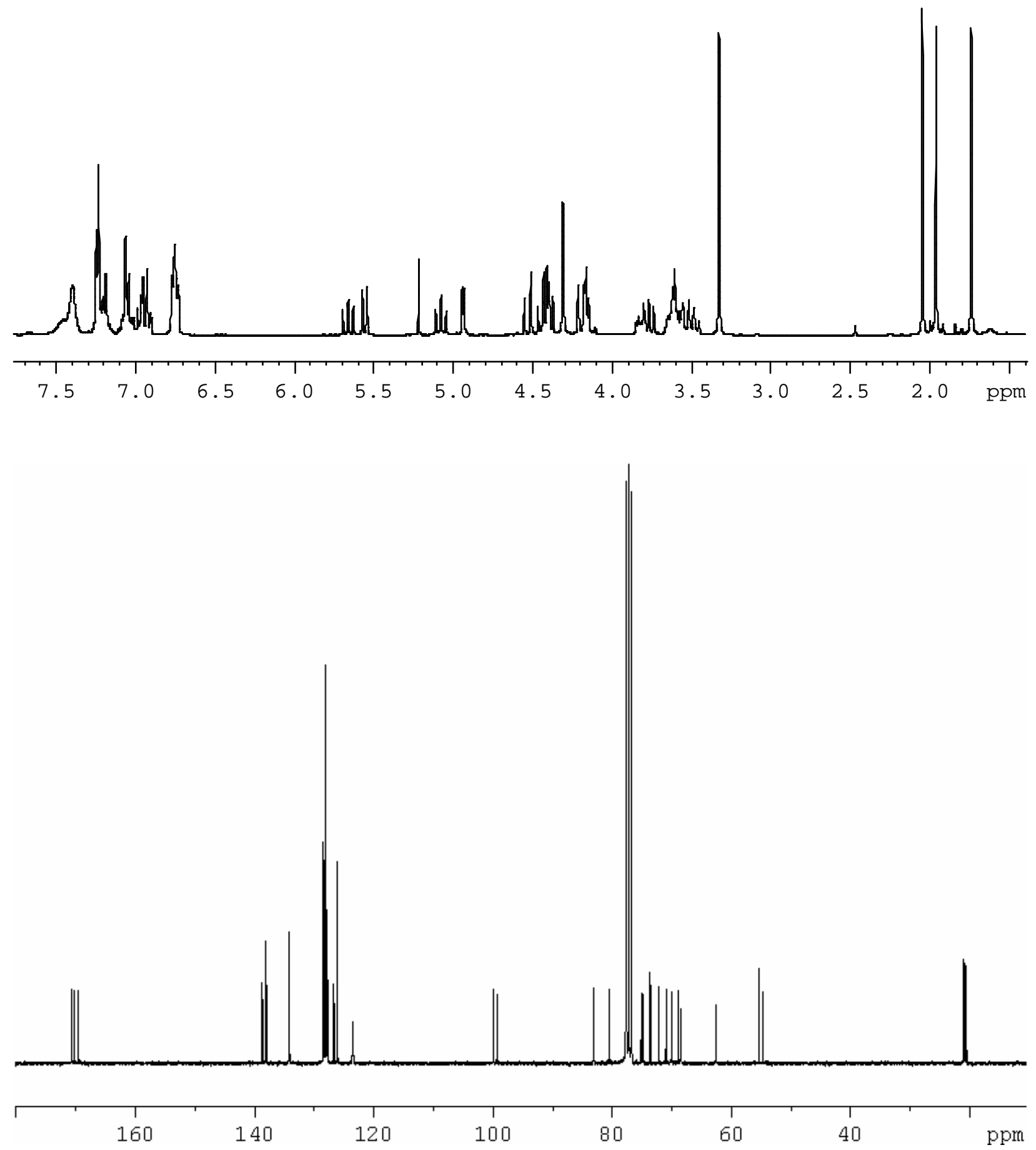

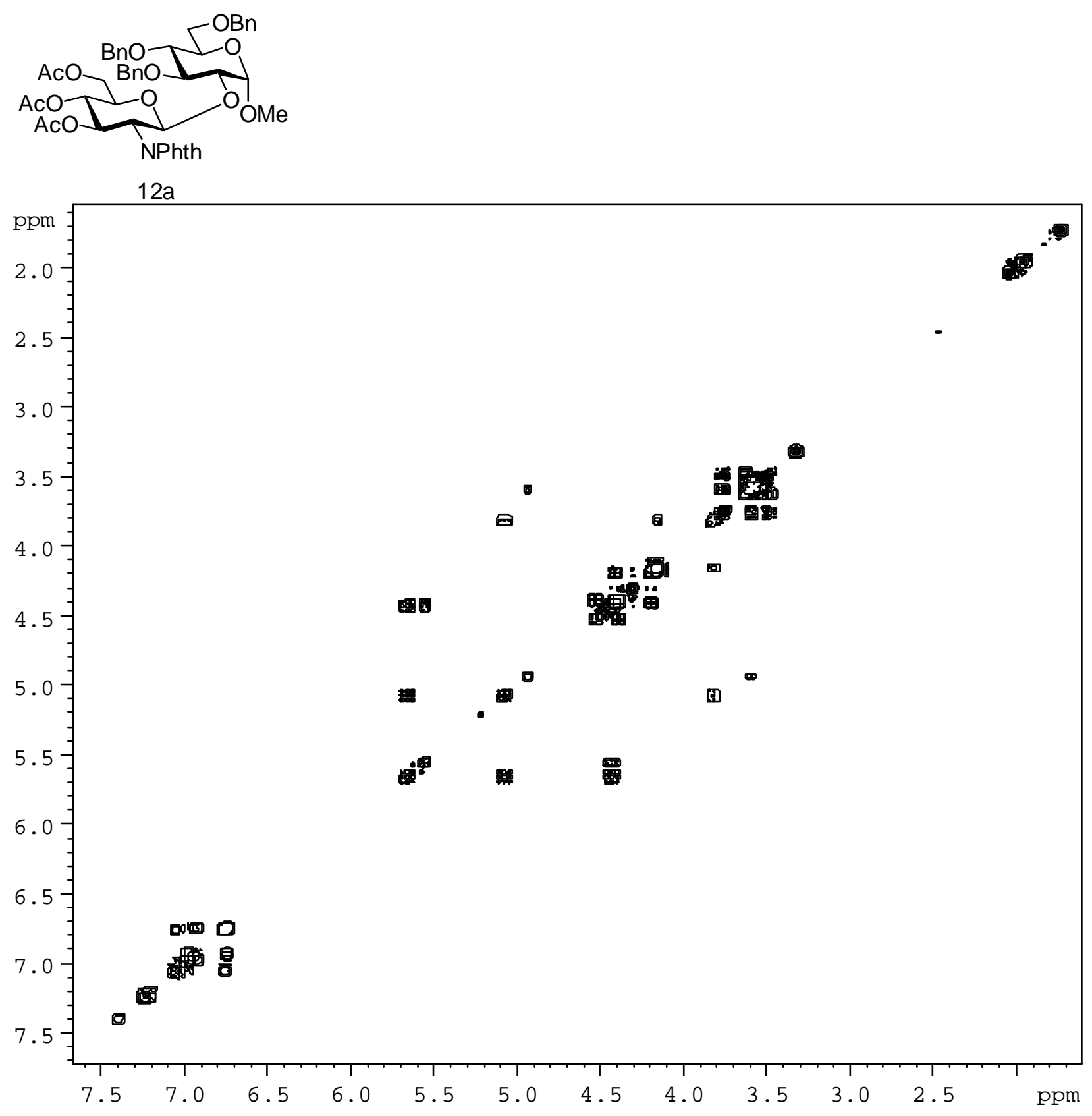


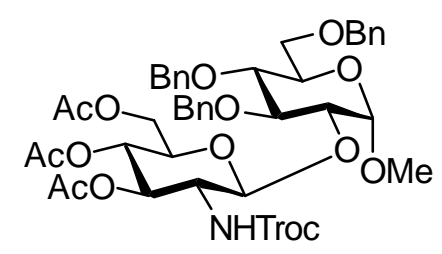

$12 \mathrm{~b}$
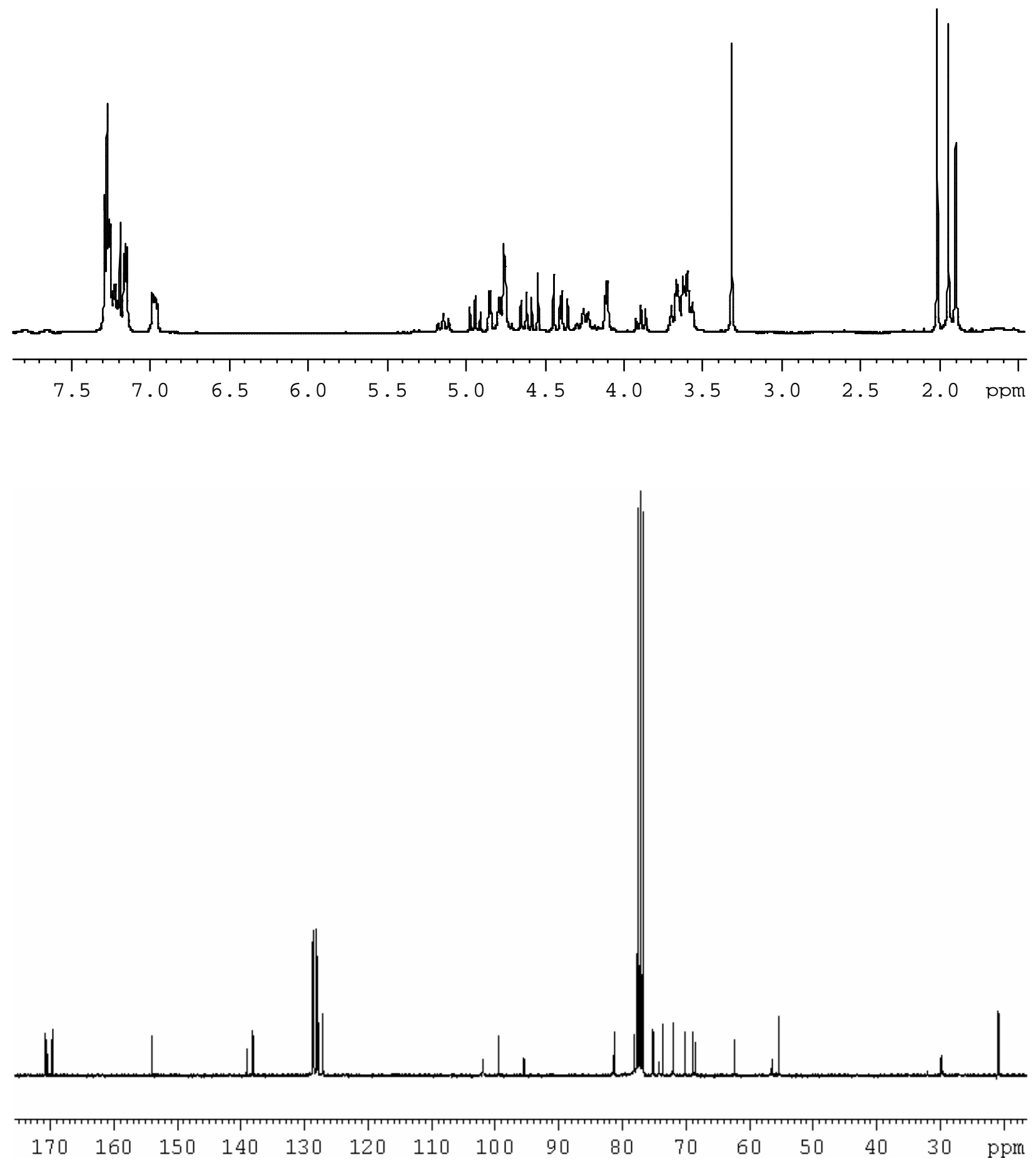

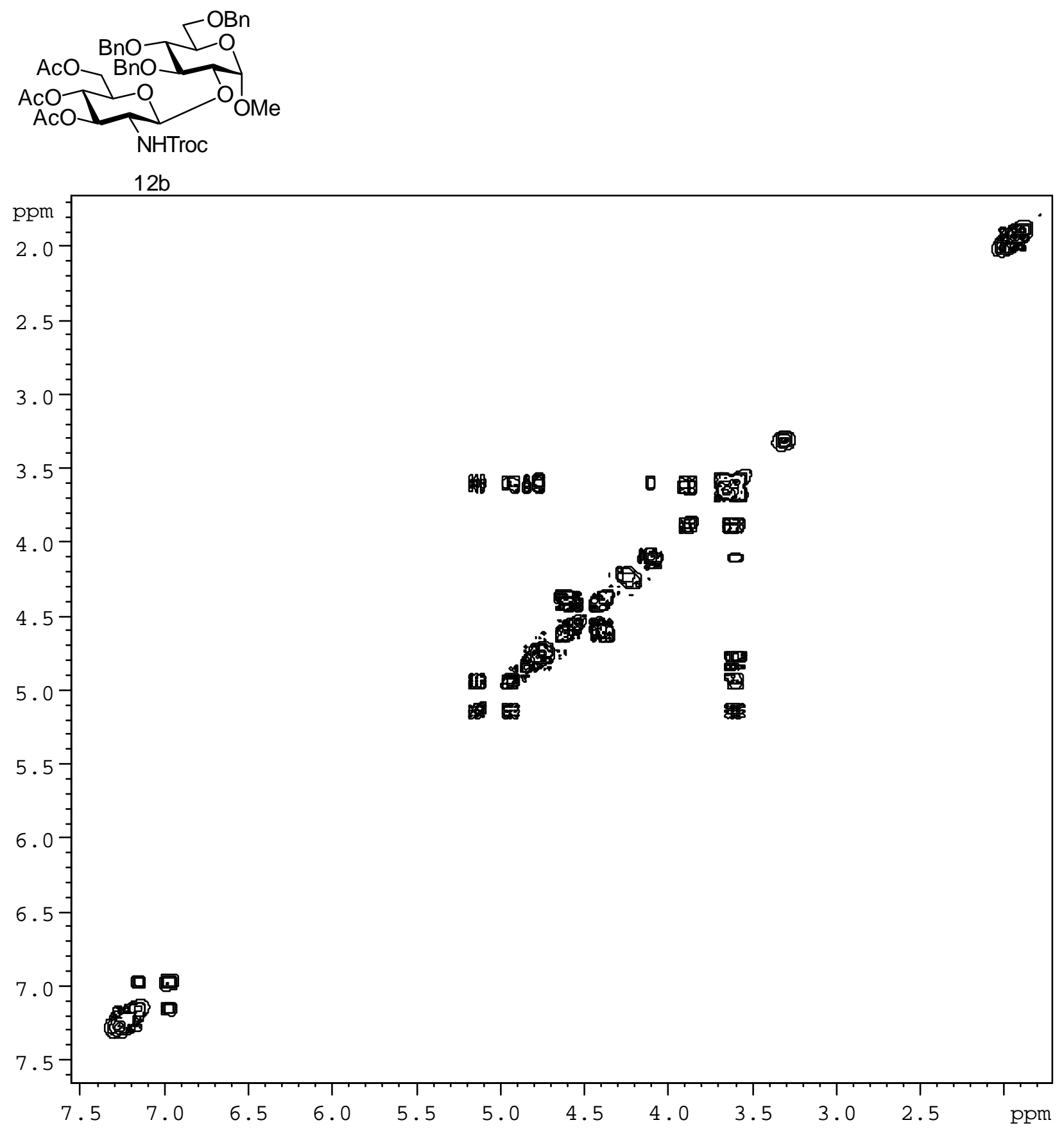


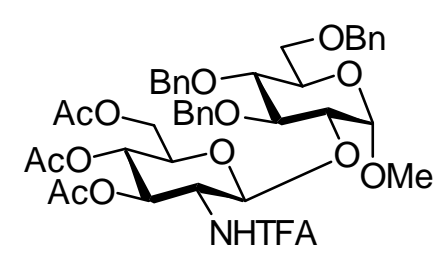

$12 \mathrm{c}$
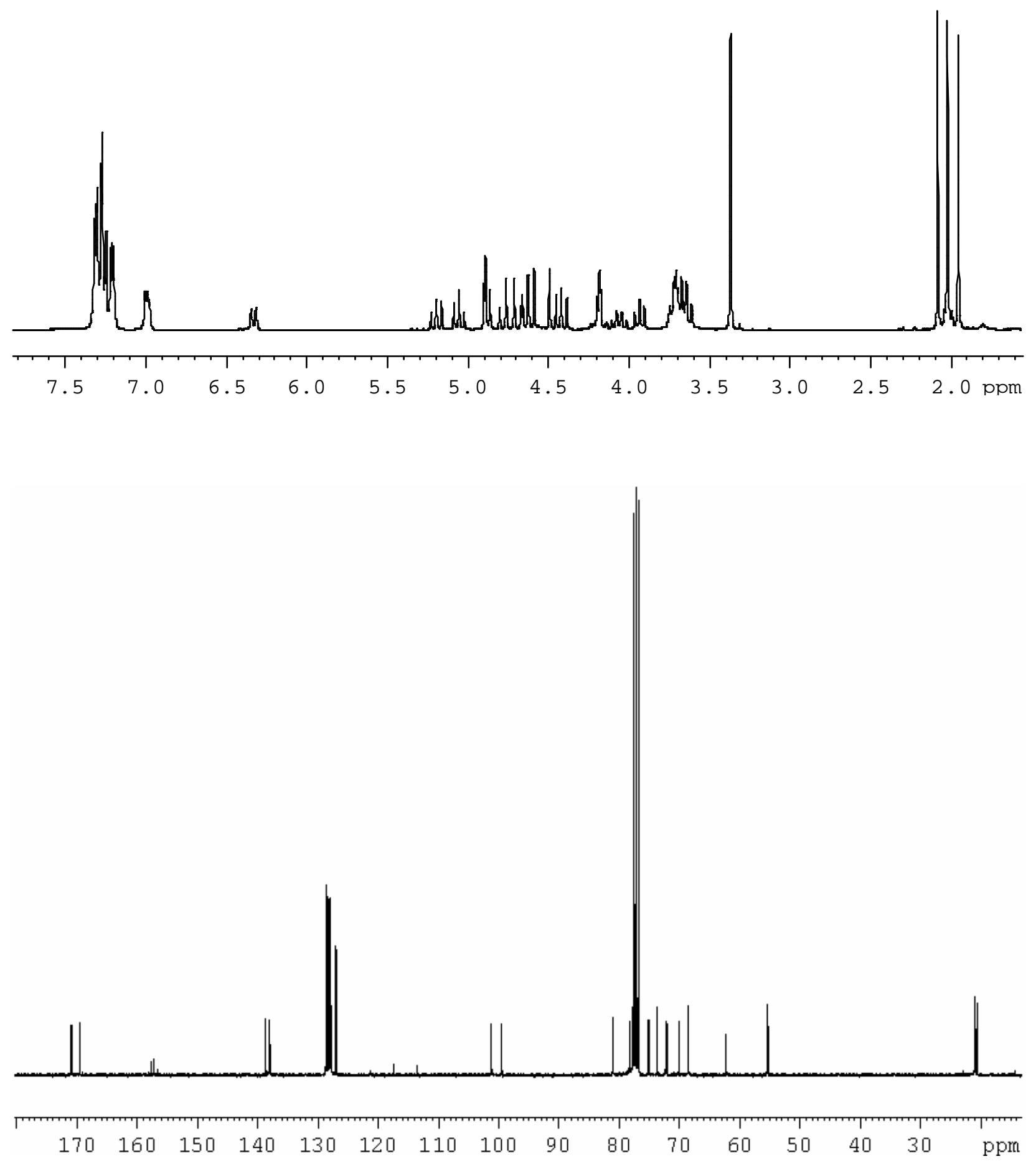

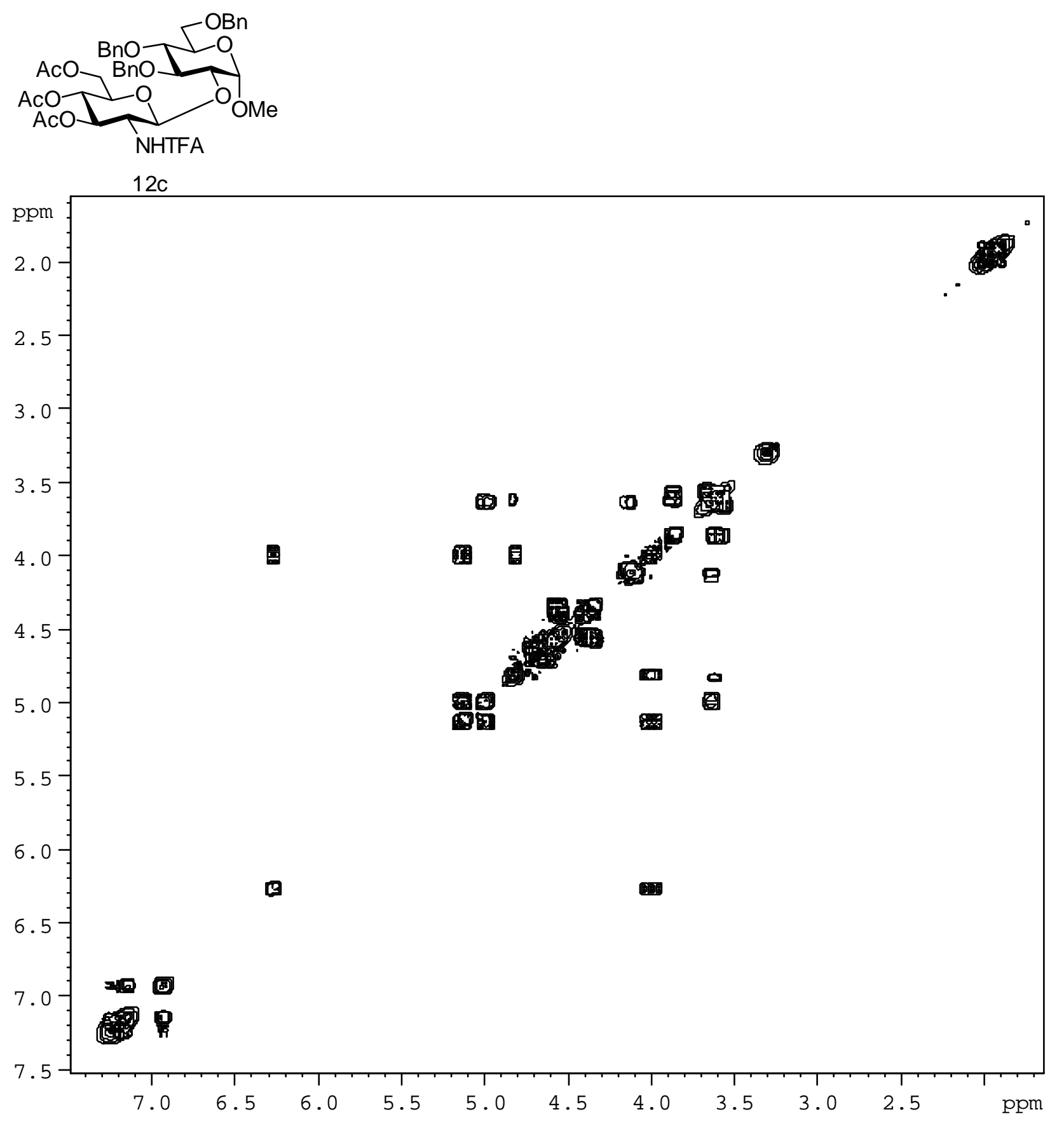


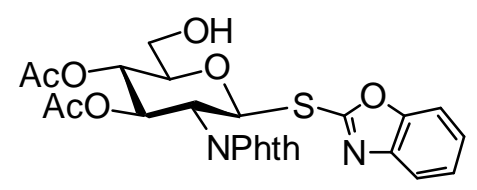

13
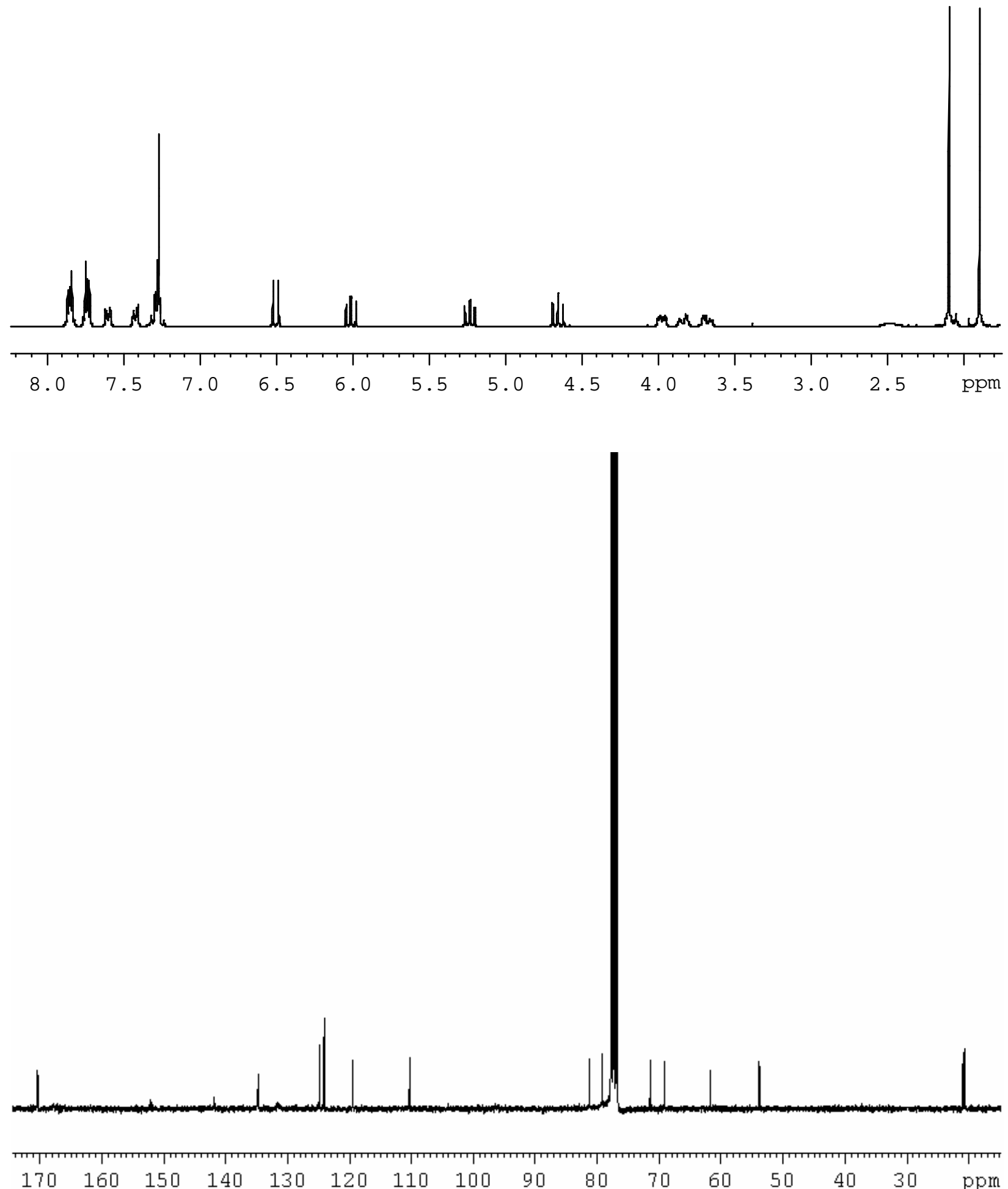

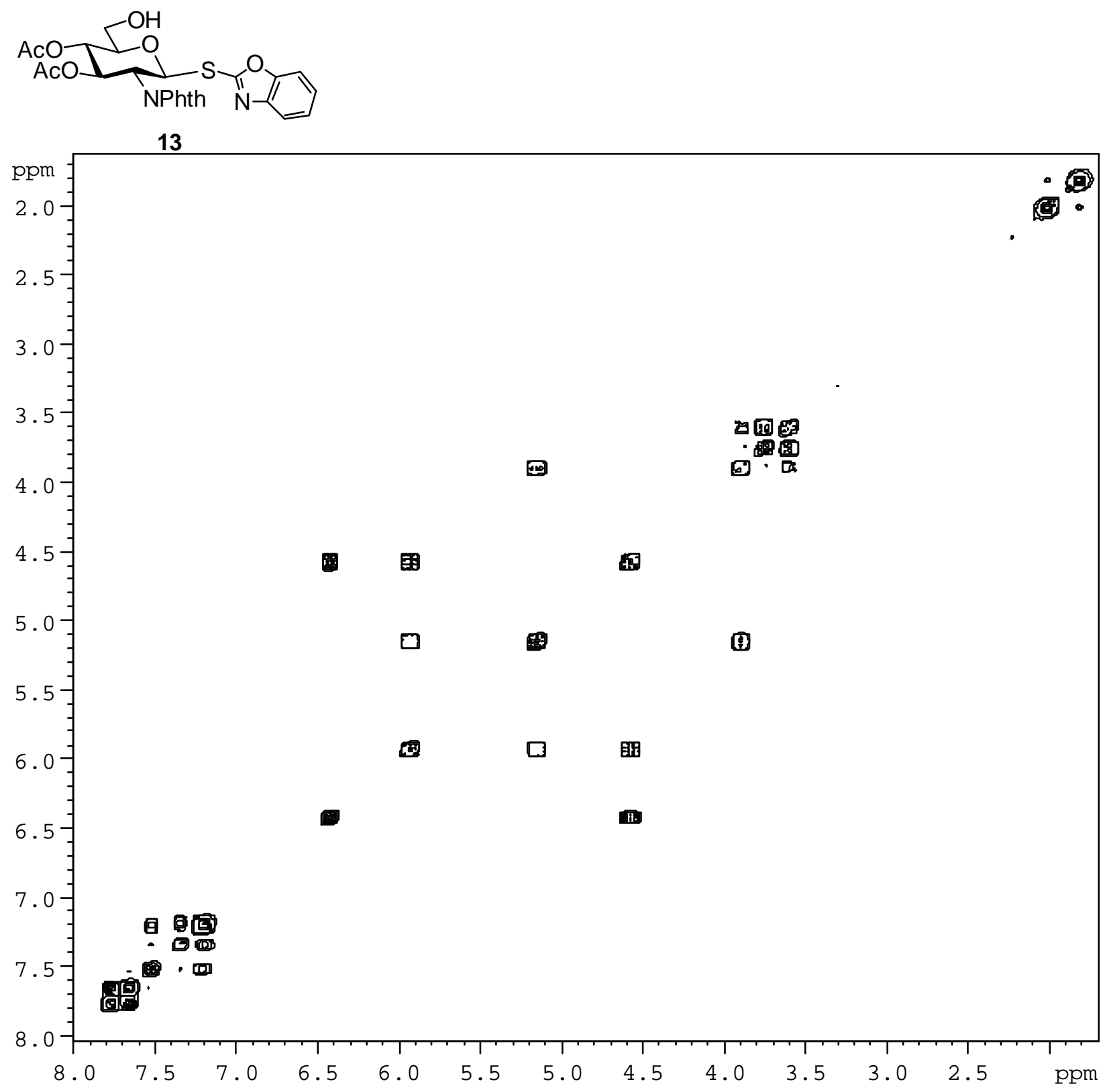

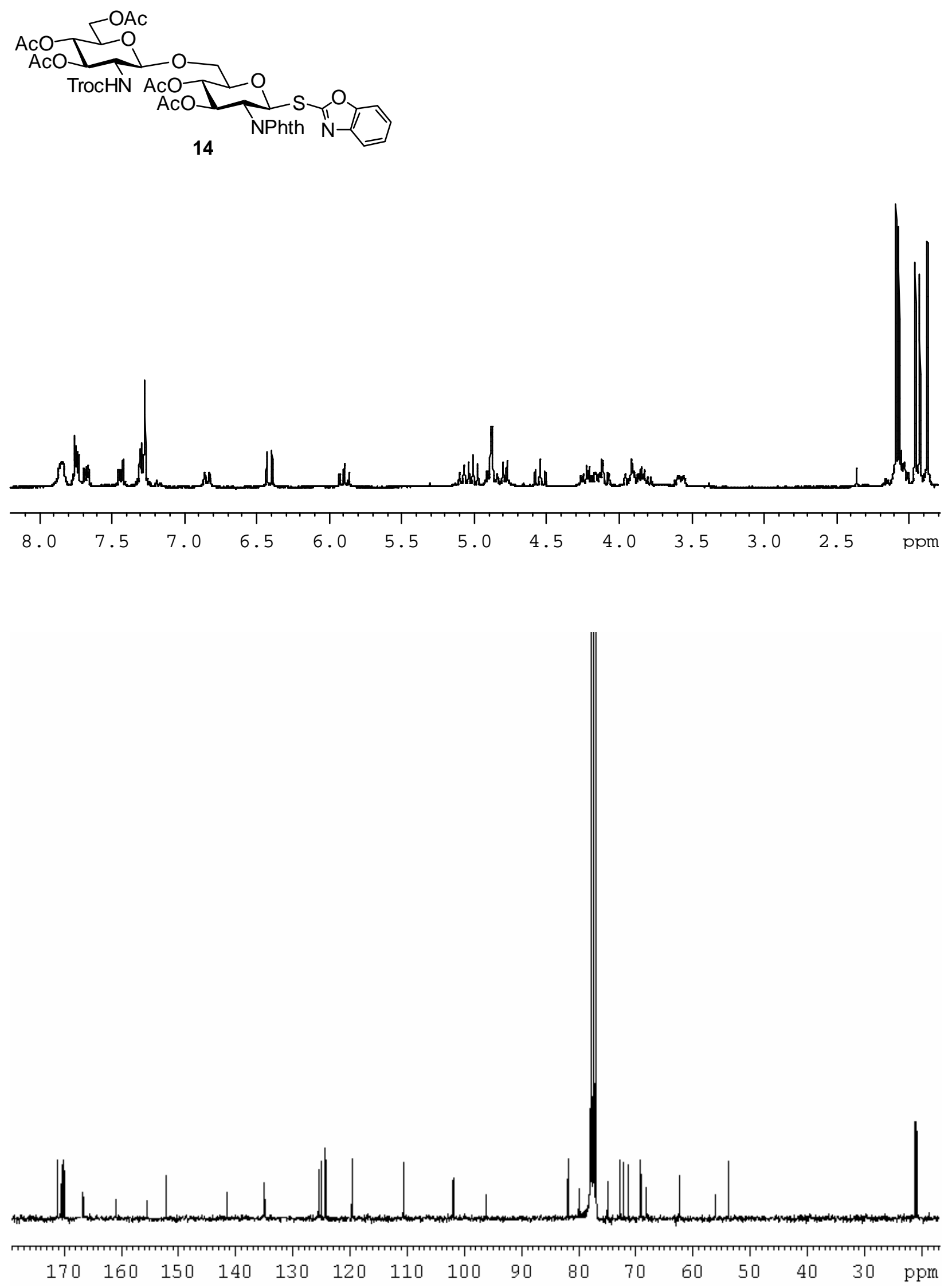


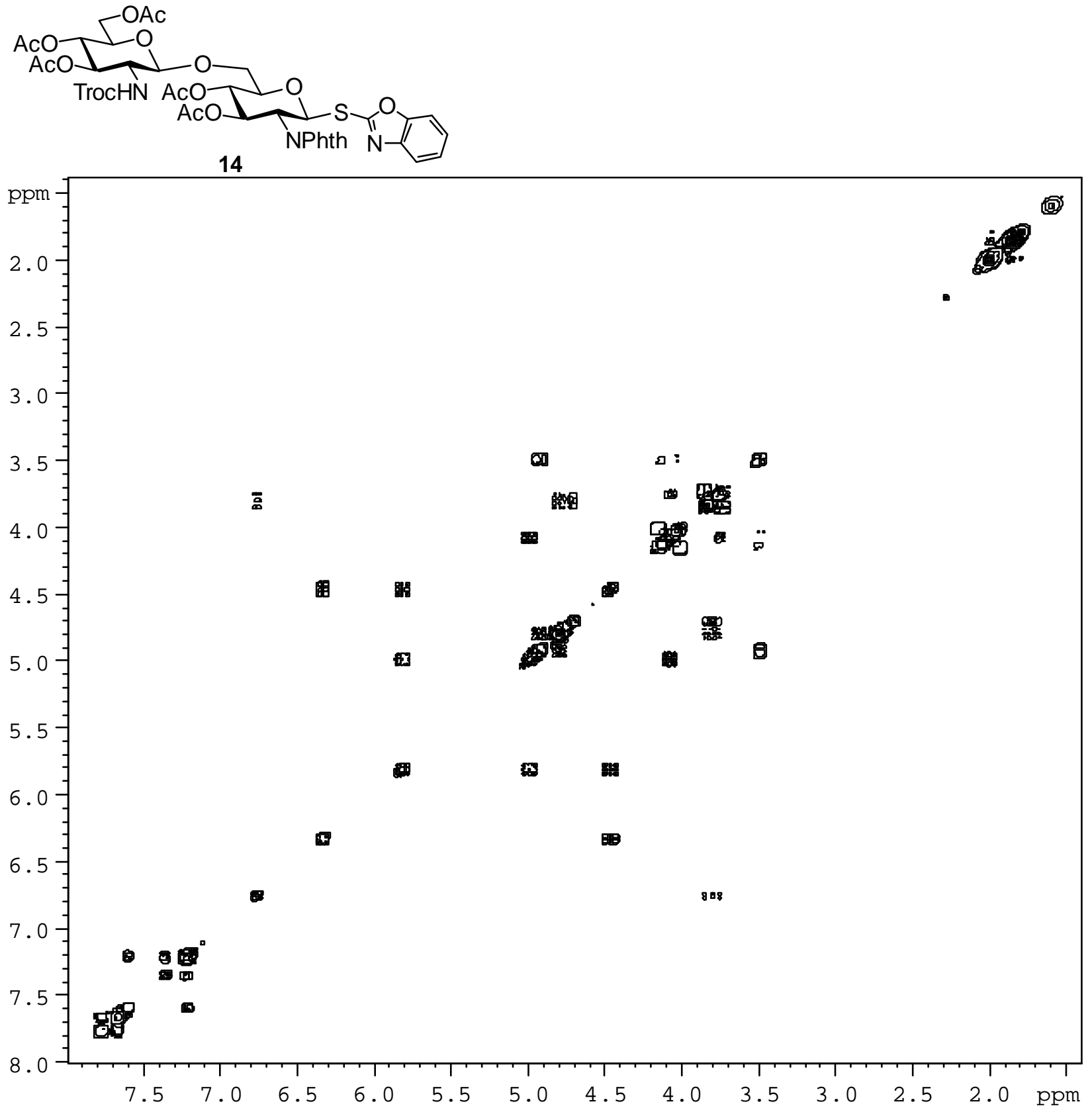

S-54 

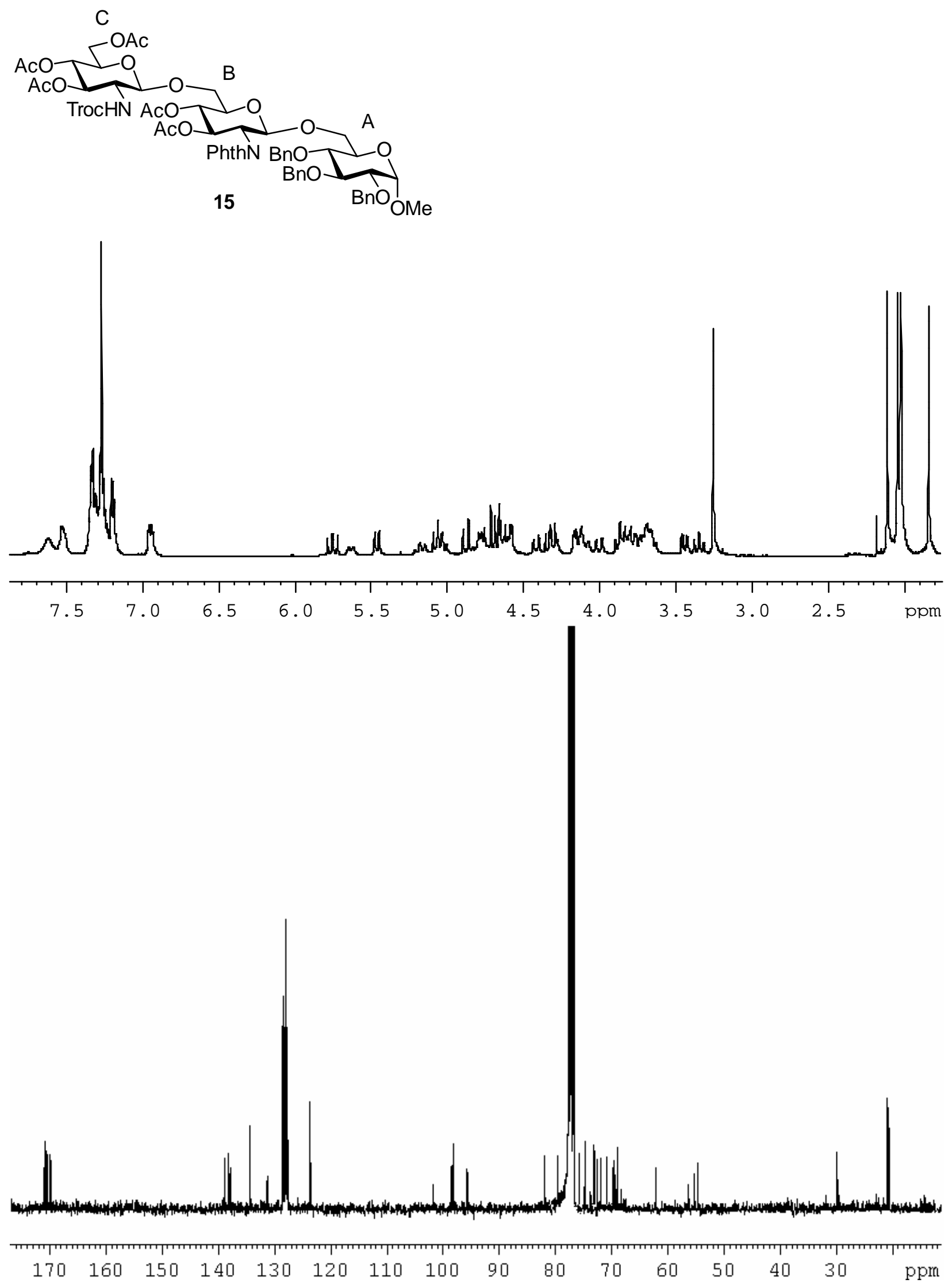


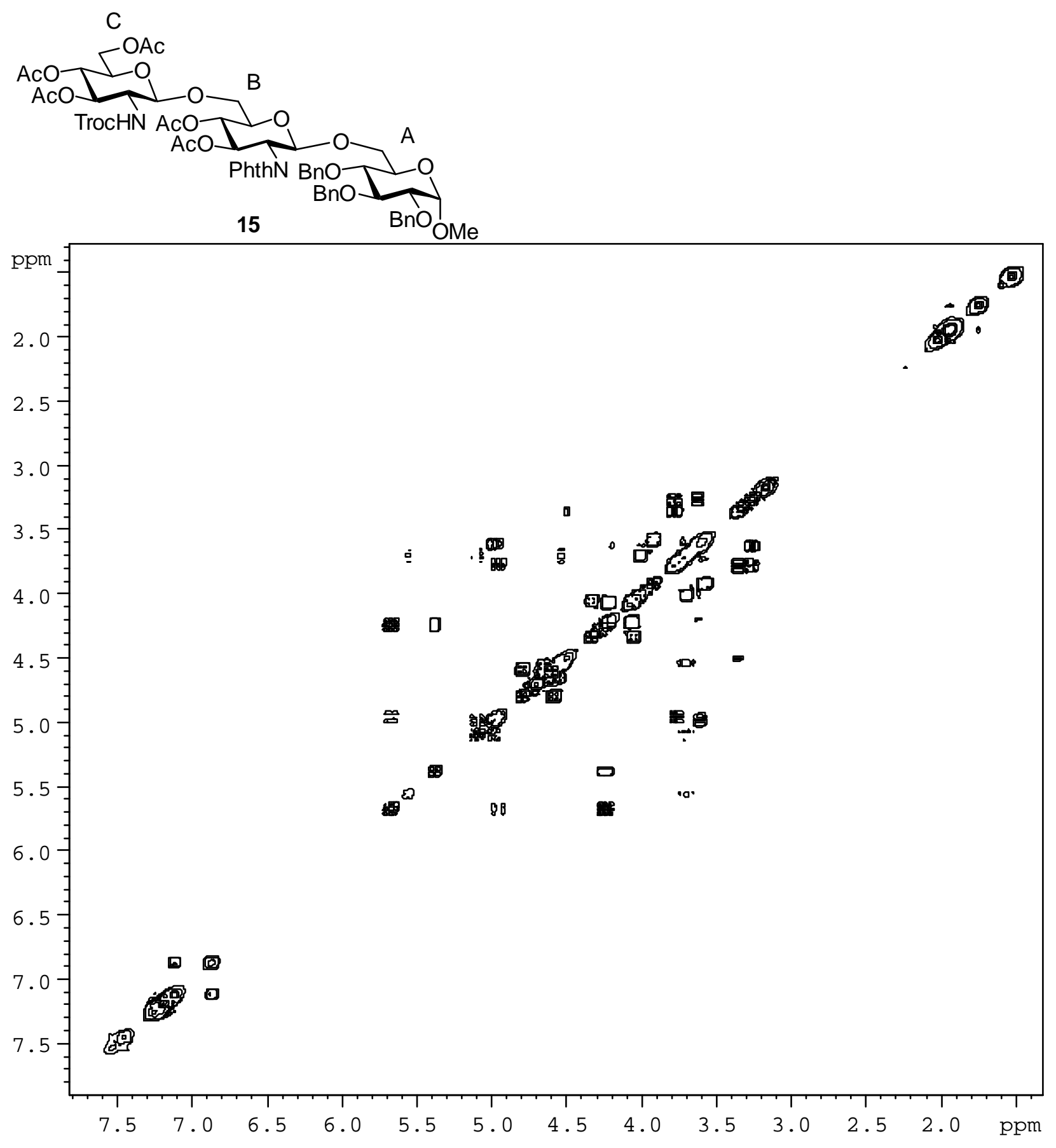

